\title{
ON THE STRENGTH OF RAMSEY'S THEOREM FOR PAIRS
}

\author{
PETER A. CHOLAK, CARL G. JOCKUSCH, JR., AND \\ THEODORE A. SLAMAN
}

\begin{abstract}
We study the proof-theoretic strength and effective content of the infinite form of Ramsey's theorem for pairs. Let $R T_{k}^{n}$ denote Ramsey's theorem for $k$-colorings of $n$-element sets, and let $R T_{<\infty}^{n}$ denote $(\forall k) R T_{k}^{n}$. Our main result on computability is: For any $n \geq 2$ and any computable (recursive) $k$-coloring of the $n$-element sets of natural numbers, there is an infinite homogeneous set $X$ with $X^{\prime \prime} \leq_{T} 0^{(n)}$. Let $I \Sigma_{n}$ and $B \Sigma_{n}$ denote the $\Sigma_{n}$ induction and bounding schemes, respectively. Adapting the case $n=2$ of the above result (where $X$ is low 2 ) to models of arithmetic enables us to show that $R C A_{0}+I \Sigma_{2}+R T_{2}^{2}$ is conservative over $R C A_{0}+I \Sigma_{2}$ for $\Pi_{1}^{1}$ statements and that $R C A_{0}+I \Sigma_{3}+R T_{<\infty}^{2}$ is conservative over $R C A_{0}+I \Sigma_{3}$ for arithmetic statements. It follows that $R C A_{0}+R T_{2}^{2}$ does not imply $B \Sigma_{3}$. We show in contrast that $R C A_{0}+R T_{<\infty}^{2}$ does imply $B \Sigma_{3}$, and so $R T_{<\infty}^{2}$ is strictly stronger than $R T_{2}^{2}$ over $R C A_{0}$.
\end{abstract}

\section{INTRODUCTION}

Ramsey's theorem was discovered by Ramsey [1930] and used by him to solve a decision problem in logic. Subsequently it has been an important tool in logic and combinatorics.

\section{Definition 1.1.}

(i) $[X]^{n}=\{Y \subseteq X:|Y|=n\}$.

(ii) A $k$-coloring $\mathcal{C}$ of $[X]^{n}$ is a function from $[X]^{n}$ into a set of size $k$.

(iii) A set $H \subseteq X$ is homogeneous for a $k$-coloring $\mathcal{C}$ of $[X]^{n}$ if $\mathcal{C}$ is constant on $[H]^{n}$, i.e. all $n$-element subsets of $H$ are assigned the same color by $\mathcal{C}$.

1991 Mathematics Subject Classification. Primary 03F35 03C62 03D30 03D80.

Key words and phrases. Ramsey's Theorem, conservation, reverse mathematics, recursion theory, computability theory.

Research partially supported NSF Grants DMS-96-3465 (Cholak), DMS-9503398 and DMS-98-03073 (Jockusch), and DMS-97-96121 (Slaman). 
Ramsey's Theorem. For all $k$ and $n$, every $k$-coloring of $[\mathbb{N}]^{n}$ has an infinite homogeneous set.

An extensive treatment of Ramsey's Theorem, emphasizing its finite version, may be found in Graham, Rothschild and Spencer [1980], where many related results and applications are also discussed.

There are (at least) two ways to use the tools of mathematical logic to analyze Ramsey's theorem. One is via computability theory (or equivalently recursion theory): Study the complexity (in terms of the arithmetical hierarchy or degrees) of infinite homogeneous sets for a coloring $\mathcal{C}$ relative to that of $\mathcal{C}$. (For simplicity, we can assume that $\mathcal{C}$ is computable (recursive) and relativize.) The other is via reverse mathematics: Study the proof-theoretic strength of Ramsey's theorem (and its natural special cases) as a formal statement in second order arithmetic.

There has been much work done along these lines. For example, consider the independent work by Jockusch [1972], Seetapun, and Slaman (see Seetapun and Slaman [1995]). Our task in this paper is to review briefly the work that has been done and further this analysis.

Before getting into details we mention two themes in this work that we would like to make explicit. The first is that results in computability theory are sometimes the forerunners of results in reverse mathematics. This is certainly the case for Weak König's Lemma and almost all versions of Ramsey's Theorem. The second theme is the use of paths through trees, more specifically Weak König's Lemma, the Low Basis Theorem, and Scott sets. Almost all of our results use one or more of these three items in its statement or proof. Whether this use is necessary is unknown. In Section 2, there is a brief summary of previous work on the analysis of König's Lemma and the infinite form of Ramsey's Theorem in terms of computability theory and of reverse mathematics.

Our starting point is the following result, which refutes an old conjecture of Jockusch (see Jockusch [1972, Corollary 4.7] or the second paragraph after Theorem 2.5.

Theorem 3.1. For any computable coloring of the unordered pairs of natural numbers with finitely many colors, there is an infinite low homogeneous set $X$, i.e., $X^{\prime \prime} \leq_{T} 0^{\prime \prime}$.

The proof is not simply an effectivization of the standard proof of Ramsey's Theorem. Instead, the first step is to restrict the given computable coloring to a low $2 \mathrm{r}$-cohesive set $A$, which exists by Jockusch and Stephan [1993], Theorem 2.5. Since for any $a$ the color of the 
pair $\{a, b\}$ is independent of $b$ for sufficiently large $b \in A$, the coloring induces a coloring of $[A]^{1}$ which is $\Delta_{2}^{0, A}$. Then the relativization to $A$ of the following new result easily yields the desired infinite $l_{0}$ homogeneous set.

Theorem 3.7. If $A_{1}, A_{2}, \ldots, A_{n}$ are $\Delta_{2}^{0}$ sets and $\cup_{i=1}^{n} A_{i}=\mathbb{N}$, then some $A_{i}$ has an infinite low subset. $^{2}$

We give two proofs of the above result, with the common elements of the two proofs presented in Section 3 .

The first proof, which was our original proof, is technically easier since it uses the Low Basis theorem to reduce the problem of controlling the second jump of the constructed set to the easier problem of controlling the first jump of the constructed set. (A similar approach was used in Jockusch and Stephan [1993] to construct a low $2 \mathrm{r}-$-cohesive set.) In Section 4 we will present this first proof and also, for the con-

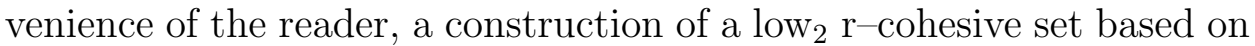
control of the first jump. This "first jump" method also yields interesting additional information on the jumps of degrees of homogeneous sets (see Section 12).

Our second proof, which will be presented in Section 5, is more direct and also somewhat more complicated. It proceeds by direct control of the second jump of the constructed set. It gives no additional information on degrees of homogeneous sets, and the reader interested only in the computability aspect of this paper could well omit reading it. We also give a construction of a low $_{2}$ r-cohesive set using direct control of the second jump. As above, this is more complicated than the construction used in Jockusch and Stephan [1993]. The reason for giving these more involved constructions is that they seem to be more suitable to adapting to models of arithmetic to obtain results in reverse mathematics as described below.

In Section 6, we quickly introduce the reader to second order arithmetic. (The reader unfamiliar with second order arithmetic may want to start there.) A listing of the needed statements of second order arithmetic and the relationships among them can be found in Section 7 . We will assume that the reader is somewhat familiar with computability theory; a good introduction is Soare [1987]. In Section 8, we discuss some results concerning Weak König's Lemma; a reasonable portion of this section was known previously but much of it is new.

Sections 911 present our conservation theorems for Ramsey's Theorem for pairs and related principles. Let $X \rightarrow[X]_{k}^{n}$ be the statement "every $k$-coloring of $[X]^{n}$ has an infinite homogeneous set." Thus, Ramsey's Theorem states for all $k$ and $n, \mathbb{N} \rightarrow[\mathbb{N}]_{k}^{n}$. $R T_{k}^{n}$ is the statement 
in the language of second order arithmetic "for all $k$-colorings of $[\mathbb{N}]^{n}$ there is an infinite homogeneous set $H$."

We adapt the forcing used in the "second jump" constructions to models of arithmetic to produce a notion of forcing for adding infinite homogeneous sets to models of second order arithmetic while preserving the appropriate level of induction. We were led to this notion of forcing by a conjecture of Slaman (see Conjecture 2.10 or Seetapun and Slaman [1995]). (We do not know how to do this for the "first jump" proofs.) Using this notion of forcing we obtain the following result.

Theorem 10.1. $R C A_{0}+I \Sigma_{2}+R T_{2}^{2}$ is $\Pi_{1}^{1}$-conservative over $R C A_{0}+I \Sigma_{2}$.

This means that any $\Pi_{1}^{1}$ statement provable from $R C A_{0}+I \Sigma_{2}+R T_{2}^{2}$ is provable from just $R C A_{0}+I \Sigma_{2}$. The following corollary answers the second part of Seetapun and Slaman [1995, Question4.3].

Corollary 1.2. $R T_{2}^{2}$ does not imply PA over $R C A_{0}$.

This improves Seetapun's result (see Seetapun and Slaman [1995]) that $R T_{2}^{2}$ does not imply $A C A_{0}$ over $R C A_{0}$. In the same paper, Slaman showed in Theorem 3.6 that $R C A_{0}+R T_{2}^{2}$ is not $\Pi_{4}^{0}$-conservative over $R C A_{0}$.

It turned out that our proof-theoretic results (but not the corresponding results in computability theory) are sensitive to whether our colorings use two colors or an arbitrary finite number of colors.

$X \rightarrow[\omega]_{<\infty}^{n}$ is the statement that "for all $k$, for all $k$-colorings of $[X]^{n}$ there is an infinite homogeneous set." Ramsey's Theorem implies for all $k$ and $n, \mathbb{N} \rightarrow[\omega]_{<\infty}^{n} . R T_{<\infty}^{n}$ is the statement in the language of second order arithmetic "for all $k$, for all $k$-colorings of $[\mathbb{N}]^{n}$ there is an infinite homogeneous set $H . "$

Using a modification of the above mentioned notion of forcing (working over a Scott set), we proved the following result.

Theorem 11.1. $R C A_{0}+I \Sigma_{3}+R T_{<\infty}^{2}+$ Weak König's Lemma is conservative over $R C A_{0}+I \Sigma_{3}$ for arithmetic statements.

Thus any arithmetic statement provable from $R C A_{0}+I \Sigma_{3}+R T_{<\infty}^{2}+$ Weak König's Lemma is provable from just $R C A_{0}+I \Sigma_{3}$. So $R T_{<\infty}^{2}$ does not imply $P A$ over $R C A_{0}$. In addition, we improve some work of Mytilinaios and Slaman [1994] to obtain the following result.

Corollary 11.5. $R C A_{0}+R T_{<\infty}^{2} \vdash B \Sigma_{3}$.

Since $I \Sigma_{2}$ is stricter weaker than $B \Sigma_{3}$ (over $R C A_{0}$ ) (see Kaye [1991] or Hájek and Pudlák [1993]), it follows that $R T_{2}^{2}$ does not imply $R T_{<\infty}^{2}$ over $R C A_{0}$. 
Theorem 3.1 also leads to further results on computability and Ramsey's theorem which are covered in Section 12 . For example, the following result is obtained for colorings of $n$-tuples:

Theorem 12.1. For each $n \geq 2$ and each computable 2-coloring of $[\mathbb{N}]^{n}$, there is an infinite homogeneous set $A$ with $A^{\prime \prime} \leq_{T} 0^{(n)}$.

Other results on computability include a characterization of the degrees $\mathbf{d}$ such that every computable 2 -coloring of $[\mathbb{N}]^{2}$ has an infinite homogeneous set with jump of degree $\mathbf{d}$ (Corollary 12.6) and a result combining cone avoidance with some control of the first jump of an infinite homogeneous set (Theorem 12.2).

We do not succeed in obtaining a complete understanding of the proof-theoretic strength of Ramsey's theorem for pairs or of the degrees of infinite homogeneous sets for computable 2-colorings of pairs. A number of open questions are listed in the final section.

One theme of this paper is the close relationship between results in computability theory and results in reverse mathematics. Of course, this relationship has turned up in many other contexts, too. We hope that readers will be interested in both aspects of this paper. However, the reader interested only in the computability aspect need read only Sections 1,4 and 12,13 . The reader interested only in the reverse mathematics aspect need read only Sections $1,3,5,11$, and 13 .

\section{HistoRY}

This paper continues a stream of work on analysis of the effective content of mathematical statements and corresponding work on the strength of these statements within second order arithmetic. Here we give a brief summary of some closely related previous work in this area. For further information, see Simpson [1999].

See Section 6 for a summary of the subsystems of second-order arithmetic we shall consider. More extensive treatments may be found in Friedman [1975] and Simpson [1999]. Here we briefly remind the reader that our base theory is $R C A_{0}$, which is based on algebraic axioms and the schemes of $\Delta_{1}^{0}$-comprehension and $\Sigma_{1}$ induction. The $\omega$-models of $R C A_{0}$ are those nonempty subsets of $P(\mathbb{N})$ closed under $\oplus$ and closed downwards under $\leq_{T}$. The stronger system $A C A_{0}$ includes the arithmetic comprehension scheme $A C A$. The $\omega$-models of $A C A_{0}$ are the $\omega$ models of $R C A_{0}$ which are closed under the jump operation.

Before getting to the analysis of Ramsey's theorem, we consider König's lemma, which in fact will play an important role in this paper. Of course, König's lemma is the assertion that any infinite, finite branching tree has an infinite path. We shall actually be concerned 
with the case where there is an effective bound on the branching. Let Weak König's Lemma be the assertion that every infinite tree in $2^{<\omega}$ has an infinite path, and let $W K L_{0}$ be $R C A_{0}+$ Weak König's Lemma .

It is easy to construct infinite computable trees in $2^{<\omega}$ with no infinite computable paths, using, for example, the existence of disjoint computably enumerable sets which are not separable by any computable set. From this it follows that Weak König's Lemma cannot be proved in $R C A_{0}$.

In the other direction G. Kreisel proved the Kreisel basis theorem: Any infinite computable tree in $2^{<\omega}$ has an infinite path computable from the halting problem $0^{\prime}$. The corresponding result in reverse mathematics, due to Steve Simpson, is that Weak König's Lemma can be proved in the system $A C A_{0}$.

Theorem 2.1 (Jockusch and Soare [1972]). For any noncomputable sets $C_{0}, C_{1}, \ldots$ and any infinite computable tree $T \subseteq 2^{<\omega}$ there is an infinite path $f$ through $T$ such that $(\forall i)\left[C_{i} \not_{T} f\right]$.

The corresponding result in reverse mathematics is the following.

Corollary 2.2 (Simpson [1999]). Arithmetic Comprehenson is not provable in $W K L_{0}$.

The following result, due to Jockusch and Soare, is known as the Low Basis Theorem.

Theorem 8.1. Jockusch and Soare [1972], Theorem 2.1 Any infinite computable tree in $2^{<\omega}$ has an infinite low path $f$, i.e., $f^{\prime} \leq_{T} 0^{\prime}$.

The forcing conditions used to prove the above result are trees, and this forcing was adapted by Leo Harrington to obtain the following result.

Theorem 8.4. (Harrington, see Simpson [1999]). Any $\Pi_{1}^{1}$ statement provable from $W K L_{0}$ is provable from just $R C A_{0}$.

Since $\Sigma_{2}$ induction (without parameters) is not provable in $R C A_{0}$ (see Hájek and Pudlák [1993]), it follows that $\Sigma_{2}$-induction is not provable from $W K L_{0}$.

We now consider the analysis of Ramsey's Theorem. The first result concerning the effective content of the infinite form of Ramsey's Theorem was obtained in Specker [1971].

Theorem 2.3 (Specker [1971]). There is a computable 2-coloring of $[\mathbb{N}]^{2}$ with no infinite computable homogeneous set.

Since the family of computable sets is an $\omega$-model of $R C A_{0}$, there is an immediate corollary. 
Corollary 2.4 (Specker [1971]). $R T_{2}^{2}$ is not provable in $R C A_{0}$.

The next work in the area was due to Jockusch.

Theorem 2.5 (Jockusch [1972]).

( $i$ ) For any $n$ and $k$, any computable $k$-coloring of $[\mathbb{N}]^{n}$ has an infinite $\Pi_{n}^{0}$ homogeneous set.

(ii) For any $n \geq 2$, there is a computable 2 -coloring of $[\mathbb{N}]^{n}$ which has no infinite $\Sigma_{n}^{0}$ homogeneous set.

(iii) For any $n$ and $k$ and any computable $k$-coloring of $[\mathbb{N}]^{n}$, there is an infinite homogeneous set $A$ with $A^{\prime} \leq_{T} 0^{(n)}$.

(iv) For each $n \geq 2$, there is a computable 2 -coloring of $[\mathbb{N}]^{n}$ such that $0^{(n-2)} \leq_{T}$ A for each infinite homogeneous set $A$.

The first part was proved by induction on $n$, using a finite injury priority argument for the case $n=2$ and the Low Basis theorem for the induction step. Note that there is a slight gap between the third and fourth items.

Fix a 2-coloring of $[\mathbb{N}]^{2}$. The third item tells us that there is an infinite homogeneous set $A$ such that $A^{\prime} \leq_{T} \mathbf{0}^{\prime \prime}$. Jockusch [1972] conjectured that this cannot be improved to give the existence of an infinite homogeneous set $A$ such that $A^{\prime \prime} \leq_{T} 0^{\prime \prime}$. By Theorem 3.1, we now know that this conjecture was false.

Simpson obtained results in reverse mathematics which are related to Theorem 2.5.

Corollary 2.6 (Simpson [1999]).

(i) For each $n \geq 3$ and $k \geq 2$ (both $n$ and $k$ fixed), the statements $R T_{k}^{n}$ and $R T_{<\infty}^{n}$ are equivalent to $A C A_{0}$ over $R C A_{0}$.

(ii) The statement $R T$ is not provable in $A C A_{0}$.

(iii) $R T$ does not prove $A T R_{0}$.

(iv) $A T R_{0}$ proves $R T$. (Actually there are stronger results along this line in Simpson [1999].)

Sketch of the proof: $i$. Fix $n \geq 3$. A relativized version of Theorem 2.5 $i v$ "says" that any model of $R C A_{0}+R T_{2}^{n}$ must be closed under the jump operator. Hence any such model must contain all sets arithmetically definable from the reals in it.

A relativized version of Theorem $2.5 i$ "says" that every coloring of $n$ tuples has a homogeneous set which is arithmetic in the coloring. Hence in any model of $A C A_{0}$ every coloring of $n$-tuples has a homogeneous set.

ii. A relativized version of Theorem $2.5 \mathrm{iv}$ "says" that any model of $R T_{2}^{n}$ is closed under the $(n-2)$-jump. But one can find non-standard 
models of $A C A_{0}$ which are not closed under the $(n)$-jump for any nonstandard integer $n$.

iii. The family of all arithmetic sets is an $\omega$-model of $A C A_{0}+R T$. Since this is not a model of $A T R_{0}$, the claim follows.

$i v$. Any model of $A T R_{0}$ is closed under the $(n)$-jump, for any $n$ in the model. Theorem 2.5 ii "says" that every $k$-coloring of $[X]^{n}$ has a homogeneous set which is Turing reducible to $X^{(n)}$. If $X$ is in $\mathcal{M}$ then $X^{(n)}$ is in $\mathcal{M}$ and therefore a homogeneous set for the above coloring is in $\mathcal{M}$.

This is how things stood for twenty years. During that time, the strength of $R T_{2}^{2}$ remained a mystery. Sometimes this was phrased as the " $3-2$ " question: is $R T_{2}^{2}$ equivalent to $R T_{2}^{3}$ (over $R C A_{0}$ )? In ground-breaking work, D. Seetapun answered this question negatively by obtaining the following result.

Theorem 2.7 (Seetapun and Slaman [1995]). For any computable 2coloring $\mathcal{C}$ of $[\mathbb{N}]^{2}$ and any noncomputable sets $C_{0}, C_{1}, \ldots$, there is an infinite homogeneous set $X$ such that $(\forall i)\left[C_{i} \mathbb{Z}_{T} X\right]$.

This allowed Seetapun to construct an $\omega$-model of $R C A_{0}+R T_{2}^{2}$ which was not closed under the jump operator and hence deduce the following corollary.

Corollary 2.8 (Seetapun and Slaman [1995]). In $R C A_{0}, \quad R T_{2}^{2}$ does not imply $A C A_{0}$. Hence, over $R C A_{0}, R T_{2}^{2}$ is strictly weaker than $R T_{2}^{3}$.

In the same paper, Slaman obtained the following result going in the opposite direction.

Theorem 2.9 (Seetapun and Slaman [1995]). $R T_{2}^{2}$ is not $\Pi_{4}^{0}$-conservative over $R C A_{0}$.

This is what was known up to the time of our work. Note that the series of results on Ramsey's theorem for pairs is somewhat parallel to the results for Weak König's Lemma. In particular, Seetapun's Theorem 2.7 and its corollary that $R T_{2}^{2}$ does not imply $A C A_{0}$ (Corollary 2.8) are analogous, to the Jockusch-Soare cone avoidance theorem for $\Pi_{1}^{0}$-classes (Theorem 2.1) and its corollary that $W K L_{0}$ is strictly weaker than $A C A_{0}$ (Corollary 2.2), respectively. However, in this historical survey there is no analogue for Ramsey's theorem mentioned for the Low Basis Theorem and Harrington's $\Pi_{1}^{1}$ conservation theorem for $W K L_{0}$, Theorem 8.4. It is the analog between Weak König's Lemma and Ramsey's theorem, which led Slaman to make the following conjecture. 
Conjecture 2.10 (Seetapun and Slaman [1995]). Any proof that every computable 2-coloring of $[\mathbb{N}]^{2}$ has an infinite homogeneous low set should lead to a proof that $R C A_{0}+R T_{2}^{2}$ is $\Pi_{1}^{1}$-conservative over $R C A_{0}+I \Sigma_{n}$.

It is the main purpose of this paper to confirm Slaman's conjecture by supplying the analogues of the Low Basis Theorem and Harrington's $\Pi_{1}^{1}$ conservation theorem for $W K L_{0}$ for Ramsey's Theorem, namely Theorems 3.1, 10.2, and 11.1.

\section{3. $\mathrm{Low}_{2}$ Homogeneous Sets}

The goal of this section is to outline the structure of the proof of the following theorem. For reasons stated in the introduction, we will actually give two proofs of this result. The two proofs, although differing considerably in their details, will both have the structure outlined in this section.

Theorem 3.1. For any computable $k$-coloring of $[\mathbb{N}]^{2}$, there is an infinite homogeneous set $X$ which is low (i.e., $X^{\prime \prime} \leq_{T} 0^{\prime \prime}$ ).

Our proof of this theorem is somewhat indirect. The following definition will play a key role.

Definition 3.2. An infinite set $X$ is $r$-cohesive if for each computable set $R, X \subseteq^{*} R$ or $X \subseteq^{*} \bar{R}$. An infinite set is $p$-cohesive if the above holds for each primitive recursive set $R$.

Theorem 3.3 (Jockusch and Stephan [1993]). There exists a low $r$ cohesive set.

A proof of this result can be found in Jockusch and Stephan [1993], Theorem 2.5, although the proof presented there has an error which is corrected in Jockusch and Stephan [1997]. We will present a "single jump control" proof of this theorem in Section 4 and a "double jump control" proof of this theorem in Section 5 .

The reason for considering $\mathrm{r}-$ cohesive sets is that if $X$ is $\mathrm{r}-$ cohesive and $\mathcal{C}$ is a 2 -coloring of $[X]^{2}$, then the restriction of $\mathcal{C}$ to $[X]^{2}$ is stable in the sense of the following definition.

Definition 3.4. A $k$-coloring of $[X]^{2}$ is called stable if for each $a \in X$, the pair $\{a, b\}$ has a fixed color $c_{a}$ for all sufficiently large $b \in X$ (i.e., there is a $d_{a}$ such that for all $b$ greater than $d_{a}$ with $b \in X$, the color of $\{a, b\}$ is $c_{a}$ ).

Stable colorings were considered in Hummel [1994] and play a crucial role in Hummel and Jockusch [n.d.]. 
Now any computable $k$-coloring of pairs becomes stable when it is restricted to an r-cohesive set $X$. (Fix $i$. The sets $R_{c}=\{j:\{i, j\}$ has color $c\}$ are computable and partition $\mathbb{N}-\{i\}$ as $c$ ranges over the colors. Since $X$ is $\mathrm{r}-$ cohesive, there exists a color $c$ such that $X \subseteq \subseteq^{*} R_{c}$. Thus the color of $\{i, j\}$ is independent of $j$ for all sufficiently large $j \in X$.) Thus, using Theorem 3.3, if we can prove that every stable $k$-coloring of $[\mathbb{N}]^{2}$ has an infinite low $_{2}$ homogeneous set, the result for arbitrary computable $k$-colorings of $[\mathbb{N}]^{2}$ follows by relativization. (Any set which is $\operatorname{low}_{2}$ relative to a $\operatorname{low}_{2}$ set is $\operatorname{low}_{2}$.)

The problem of finding homogeneous sets for computable stable colorings of pairs is easily reduced, by the Limit Lemma, to the problem of finding homogeneous sets for $\Delta_{2}^{0}$ colorings of 1-tuples.

Lemma 3.5. For any computable stable $k$-coloring $\mathcal{C}$ of $[\mathbb{N}]^{2}$, there are $k$ disjoint $\Delta_{2}^{0}$ sets $A_{i}$ such that $\bigsqcup_{i<k} A_{i}=\mathbb{N}$ and any infinite subset of any $A_{i}$ computes an infinite homogeneous set for $\mathcal{C}$.

Proof. Let $A_{i}=\left\{a: \lim _{b} \mathcal{C}(\{a, b\})=i\right\}$. Suppose that $B$ is an infinite subset of $A_{i}$. Define $c_{k}$ by recursion as the least $c \in B$ such that, for all $j<k, c>c_{j}$ and $\mathcal{C}\left(\left\{c_{j}, c\right\}\right)=i$. Then $\left\{c_{i}: i \in \mathbb{N}\right\}$ is the desired infinite set $C$ such that $C$ is homogeneous for $\mathcal{C}$ and $C \leq_{T} B$.

The following results (relativized to a low 2 r-cohesive set) will complete the proof that each computable $k$-coloring of pairs has an infinite low $_{2}$ homogeneous set. (The first is a special case of the second.)

Theorem 3.6. For each $\Delta_{2}^{0}$ set $A$ there is an infinite low set $G$ which is contained in $A$ or $\bar{A}$.

Theorem 3.7. Let $\left\{A_{i}\right\}_{i<k}$ be $k$ disjoint $\Delta_{2}^{0}$ sets such that $\bigsqcup_{i<k} A_{i}=\mathbb{N}$. Then for some $k$, there is an infinite low set $G$ which is contained in $A_{k}$.

These results will be proved by "single jump control" in Section 4 and by "double jump control" in Section 5 .

Before we proceed, we should note that Theorem 3.7 follows by induction from Theorem 3.6. (Let $\left\{A_{i}\right\}_{i<k+1}$ be $k+1$ disjoint $\Delta_{2}^{0}$ sets such that $\bigsqcup_{i<k+1} A_{i}=\mathbb{N}$. Let $A=A_{k}$. Apply Theorem 3.6. If there is a low 2 subset of $A=A_{k}$, we are done. Otherwise apply the relativized (to the set $G$ ) version of the induction hypothesis (i.e., Theorem 3.7) to $\left\{A_{i} \cap G\right\}_{i<k}$.) But as we will later see (Theorem 11.4) this does not hold for models of arithmetic; the statement of Theorem 3.6. $D_{2}^{2}$, in second order arithmetic does not imply the statement of Theorem 3.7. $D_{<\infty}^{2}$. For this reason we will show, in Section 5.3, how to alter the forcing proof of Theorem 3.6 to get a proof of Theorem 3.7 . 
We now complete the proof of Theorem 3.1, assuming Theorems 3.3 and 3.7. The idea is that the existence of a $\operatorname{low}_{2} \mathrm{r}-$-cohesive set allows us to restrict attention to computable stable partitions of pairs, which are basically the same as $\Delta_{2}^{0}$ partitions of 1 -tuples, and these have infinite low $_{2}$ homogeneous sets by Theorem 3.7. In more detail, let a computable $k$-coloring $\mathcal{C}$ of $[N]^{2}$ be given. Let $X$ be a low $2 \mathrm{r}-$ cohesive set, and let $f$ be the unique increasing function with range $X$. Define an $X$-computable coloring $\mathcal{C}_{1}$ of $[N]^{2}$ by $\mathcal{C}_{1}(\{a, b\}=\mathcal{C}(\{f(a), f(b)\})$. Then, since the restriction of $\mathcal{C}$ to $[X]^{2}$ is stable, as remarked above, the coloring $\mathcal{C}_{1}$ is a stable $k$-coloring of $[N]^{2}$. By Theorem 3.5 relativized to $X$ there are sets $A_{0}, \ldots A_{k-1}$ with $\cup_{i<k} A_{i}=\mathbb{N}$ such that each $A_{i}$ is $\Delta_{2}^{0, X}$ and for any infinite set $B$ contained in any $A_{i}$, there is an infinite homogeneous set $H$ for $\mathcal{C}_{1}$ such that $H \leq_{T} X \oplus B$. By Theorem 3.7 relativized to $X$, there exists $i<k$ such that $A_{i}$ has an infinite subset $B$ with $(X \oplus B)^{\prime \prime} \leq_{T} X^{\prime \prime}$. Let $H$ be a homogeneous set for $C_{1}$ with $H \leq_{T} X \oplus B$, and let $H^{*}=f(H)$. Then $H^{*}$ is infinite and homogeneous for $\mathcal{C}$, and $\left(H^{*}\right)^{\prime \prime} \leq_{T}(X \oplus B)^{\prime \prime} \leq_{T} X^{\prime \prime} \leq_{T} 0^{\prime \prime}$, so $H^{*}$ is the desired infinite low $_{2}$ homogeneous set for $\mathcal{C}$.

\section{Constructing LOW $_{2}$ Sets By first jump CONtrol}

In this section, we prove Theorems 3.3 and 3.6 by constructing sets $A$ with $A^{\prime}$ of degree at most $\mathbf{d}$ where $\mathbf{d}$ is an appropriately chosen degree satisfying $\mathbf{d}^{\prime} \leq \mathbf{0}^{\prime \prime}$. Here "appropriately chosen" means that $\mathbf{d}>>\mathbf{0}^{\prime}$, where the relation $>>$ is defined as follows.

Definition 4.1. Let $\mathbf{a}$ and $\mathbf{b}$ be degrees. Then $\mathbf{a}>>\mathbf{b}$ means that every $\mathbf{b}$-computable $\{0,1\}$-valued partial function has a total $\mathbf{a}^{-}$ computable extension.

The notation $>>$ was defined and studied in Simpson [1977, pp. 652653]. Actually, Simpson defined $\mathbf{a}>>\mathbf{b}$ to mean that each infinite $\mathbf{b}-$ computable tree in $2^{<\omega}$ has an infinite $\mathbf{a}-$ computable path. We will see in Section 8 that this is equivalent to the above definition.

We immediately have the following implications:

$$
\mathbf{a} \geq \mathbf{b}^{\prime} \Rightarrow \mathbf{a}>>\mathbf{b} \Rightarrow \mathbf{a}>\mathbf{b}
$$

Also, for each degree $\mathbf{b}$ there is a degree $\mathbf{a}>>\mathbf{b}$ such that $\mathbf{a}^{\prime}=\mathbf{b}^{\prime}$. To prove this, consider the case where $\mathbf{b}=\mathbf{0}$ and then relativize the result to $\mathbf{b}$. Let $P$ be the class of all $\{0,1\}$-valued (total) functions $f$ such that $f(\langle e, i\rangle)=\varphi_{e}(i)$ whenever $\varphi_{e}(i) \downarrow \leq 1$. Then $P$ is a nonempty $\Pi_{1}^{0}$ subset of $2^{\omega}$, so by the Low Basis Theorem there is a low degree $\mathbf{b}$ which contains a function $f \in P$. Clearly $\mathbf{b}>>\mathbf{0}$. 
Of course, it is possible to decide the truth of a given $\Pi_{2}^{0}$ sentence in the integers using a $\mathbf{0}^{\prime \prime}$-oracle. The following lemma shows that a $\mathbf{d}$ oracle has a somewhat weaker property, which will however be sufficient for our construction. It is related to the concept of semirecursiveness studied in Jockusch [1968].

Lemma 4.2. Suppose that $\mathbf{d}>>\mathbf{0}^{\prime}$ and that $\left(\gamma_{e, 0}, \gamma_{e, 1}\right)_{e \in \omega}$ is an effective enumeration of all ordered pairs of $\Pi_{2}^{0}$ sentences of first-order arithmetic. Then there is a $\mathbf{d}$-computable $\{0,1\}$-valued (total) function $f$ such that $\gamma_{e, f(e)}$ is true whenever $\gamma_{e, 0}$ or $\gamma_{e, 1}$ is true.

Proof. Let $R(e, i, s)$ be a $0^{\prime}$-computable predicate such that, for all $e \in \omega$ and $i \leq 1, \gamma_{e . i}$ is true iff $(\forall s) R(e, i, s)$ holds. Let $\delta(e)$ be the least $s$ such that either $R(e, 0, s)$ or $R(e, 1, s)$ is false, if such an $s$ exists, and otherwise $\delta(e)$ is undefined. Let $\theta(e)=1-i$, where $i$ is minimal such that $R(e, i, \delta(e))$ is false, provided $\delta(e)$ is defined, and $\theta(e)$ is undefined otherwise. Then $\theta$ is a $\mathbf{0}^{\prime}$-computable $\{0,1\}$-valued partial function and so has a $\mathbf{b}$-computable total extension $f$. This $f$ satisfies the conclusion of the lemma.

4.1. Constructing a low 2 r-cohesive set using first jump control. The following theorem easily implies Theorem 3.3 (see Corollary 4.5.

Theorem 4.3 (Jockusch and Stephan [1993]). Suppose that the sets $R_{0}, R_{1}, \ldots$ are uniformly computable, and suppose that $\mathbf{d}>>\mathbf{0}^{\prime}$. Then there is an infinite set $G$ such that $G^{\prime}$ has degree at most $\mathbf{d}$, and for all e, either $G \subseteq{ }^{*} R_{e}$ or $G \subseteq \subseteq^{*} \overline{R_{e}}$.

Proof. The set $G$ is constructed using forcing conditions of the form $(D, L)$, where $D$ is a finite set, $L$ is an infinite computable set, and every element of $D$ is less than every element of $L$. (These are computable Mathias conditions.) A set $G$ satisfies such a condition $(D, L)$ if $D \subseteq G \subseteq D \cup L$. The requirements to be satisfied are the following:

$$
\begin{gathered}
S_{3 e}:|G| \geq e \\
S_{3 e+1}: G \subseteq^{*} R_{e} \text { or } G \subseteq^{*} \overline{R_{e}} \\
S_{3 e+2}: G^{\prime}(e) \text { is determined during the construction }
\end{gathered}
$$

An index of a condition $(D, L)$ is a pair $(a, b)$ such that $a$ is a canonical index of the finite set $D$ and $b$ is an index of the characteristic function of $L$.

The set $G$ is constructed by iterating the following lemma, which says that our requirements are $\mathbf{d}$-effectively dense. 
Lemma 4.4. For any condition $(D, L)$ and number $s$, there is a condition $\left(D^{*}, L^{*}\right)$ extending $(D, L)$ such that every set which satisfies $\left(D^{*}, L^{*}\right)$ satisfies the requirement $S_{s}$. Furthermore, an index of $\left(D^{*}, L^{*}\right)$ may be $\mathbf{d}$-effectively computed from $s$ and an index of $(D, L)$. (If $s=3 e+2$, this means that $G^{\prime}(i)$ has the same value for all $G$ satisfying $\left(D^{*}, L^{*}\right)$, and this value is computed $\mathbf{d}$-effectively.)

Proof. The case $s=3 e$ is handled by ensuring that $\left|D^{*}\right| \geq e$. To handle the case $s=3 e+1$, consider the statements " $L \cap R_{e}$ is infinite" and " $L \cap \overline{R_{e}}$ is infinite." These are $\Pi_{2}^{0}$ statements whose indices as such may be effectively computed from an index of $(D, L)$ and the value of $e$. At least one of these statements is true since $L$ is infinite. By Lemma 4.2, we may $\mathbf{d}-$-effectively select one of these statements which is true. If we select " $L \cap R_{e}$ is infinite", let $\left(D^{*}, L^{*}\right)=\left(D, L \cap R_{e}\right)$, and otherwise let $\left(D^{*}, L^{*}\right)=\left(D, L \cap \overline{R_{e}}\right)$. Clearly, $\left(D^{*}, L^{*}\right)$ is a condition with the desired property in either case and is obtained $\mathbf{d}$-effectively. Finally, consider the case where $s=3 e+2$. Ask whether there is a finite set $F$ satisfying $(D, L)$ such that $e \in F^{\prime}$. This is a $\Sigma_{1}^{0}$ question of known index, so it can be answered effectively relative to $\mathbf{0}^{\prime}$ and hence relative to $\mathbf{d}$. If there is, let $F$ be one of least index, and let $u$ be the least number which exceeds all elements of $F$ and the use of the computation showing that $e \in F^{\prime}$. Let $\left(D^{*}, L^{*}\right)=(F,\{x \in L: x>u\})$, which is obviously a condition. Then $e \in G^{\prime}$ (with the same computation) for all $G$ satisfying $\left(D^{*}, L^{*}\right)$. Finally, if there is no such $F$, let $\left(D^{*}, L^{*}\right)=(D, L)$. In this case, $e \notin G^{\prime}$ for all $G$ satisfying $(D, L)$ since convergent computations use only finitely much oracle information.

Theorem 4.3 is now deduced from Lemma 4.4 using the standard generic set construction, carried out in a $\mathbf{d}$-effective fashion. Let $\left(D_{0}, L_{0}\right)=(\emptyset, \mathbb{N})$. Given $\left(D_{i}, L_{i}\right)$, obtain $\left(D_{i+1}, L_{i+1}\right)$ by applying Lemma 4.4 to $\left(D_{i}, L_{i}\right)$ and the requirement $S_{i}$. Then $G=\cup_{i} D_{i}$ is the desired set.

Corollary 4.5 (Jockusch and Stephan [1993]). If $\mathbf{d}>>\mathbf{0}^{\prime}$, there is an $r$-cohesive set $G$ such that $G^{\prime}$ is of degree $\mathbf{d}$.

Proof. Let $\mathbf{c}$ be a low degree with $\mathbf{c}>>\mathbf{0}$, so that there is an uniformly $\mathbf{c}-$ computable sequence of sets containing all computable sets (and perhaps more). Apply Theorem 4.3 relativized to $\mathbf{c}$ to obtain an $\mathrm{r}-$ cohesive set $G_{0}$ whose degree is at most $\mathbf{d}$. By the Friedberg completeness criterion, there is a degree $\mathbf{a} \geq \operatorname{deg}(G)$ such that $\mathbf{a}^{\prime}=\mathbf{d}$, and there is an r-cohesive set $G$ of degree a by the upward closure of the r-cohesive degrees (see Jockusch [1973, Corollary 1]). 
4.2. A proof of Theorem 3.6 using first jump control. Fix a degree $\mathbf{d}>>0^{\prime}$ and a $\Delta_{2}^{0}$ set $A$. We show that there is an infinite set $X$ which is contained in or disjoint from $A$ such that $X^{\prime}$ has degree at most $\mathbf{d}$. By choosing $\mathbf{d}$ such that $\mathbf{d}^{\prime} \leq \mathbf{0}^{\prime \prime}$, we obtain a low 2 set $X$ such that $X$ is contained in or disjoint from $A$. This proves Theorem 3.6 for $k=2$ and, as mentioned in Section 3, the more general result Theorem 3.7 then follows easily by induction on $k$.

We will build a suitably generic set $G$ such that $G \cap A$ and $G \cap \bar{A}$ are both infinite and such that at least one of these sets has a $\mathbf{d}$-computable jump. We assume without loss of generality that neither $A$ nor $\bar{A}$ has an infinite low subset. The set $G$ will satisfy the following conditions:

$R_{2 e}: \quad|G \cap A| \geq e$ and $|G \cap \bar{A}| \geq e$

$R_{2\langle e, i\rangle+1}$ : Either $(G \cap A)^{\prime}(e)$ or $(G \cap \bar{A})^{\prime}(i)$ is decided during the construction.

The set $G$ is constructed using conditions $(D, L)$ where $D$ is a finite set, $L$ is an infinite low set, and every element of $D$ is less than every element of $L$. (We could have used such conditions also in the previous subsection.) Call $(a, b)$ an index of a condition $(D, L)$ if $a$ is a canonical index of $D$ and $b$ is a lowness index of $L$, i.e., $L^{\prime}=\{b\}^{K}$. Define as in the proof of Theorem 4.3 what it means for one condition to extend another and what it means for a set to satisfy a condition. The following lemma shows that the conditions forcing a given requirement $R_{e}$ to be satisfied are $\mathbf{d}$-effectively dense.

Lemma 4.6. Given a condition $(D, L)$ and a number $s$, there is a condition $\left(D^{*}, L^{*}\right)$ extending $(D, L)$ such that every set which satisfies $\left(D^{*}, L^{*}\right)$ satisfies the requirement $R_{s}$. Furthermore, an index of $\left(D^{*}, L^{*}\right)$ may be $\mathbf{d}$-effectively computed from $s$ and an index of $(D, L)$. (If $s=2\langle e, i\rangle+1$, this means that either $(G \cap A)^{\prime}(e)$ has the same value for all $G$ satisfying $\left(D^{*}, L^{*}\right)$, or $(G \cap \bar{A})^{\prime}(i)$ has the same value for all $G$ satisfying $\left(D^{*}, L^{*}\right)$. Furthermore, one can determine $\mathbf{d}$-effectively which of these two cases applies and what the common value is.)

Proof. If $s=2 e$ the result is easily proved from the assumption that $L$ has infinite intersection with $A$ and with $\bar{A}$.

Assume now that $s=2\langle e, i\rangle+1$. We now use a technique which is fundamental for this paper. We consider partitions of $L$ into pieces, each of which satisfies our requirement. However, we do not require that the pieces be infinite, since this would introduce too high a level of quantifier complexity.

Let $(\hat{D}, \hat{L})$ be a pair of sets such that $\hat{D}$ is finite and every element of $\hat{D}$ is less than every element of $\hat{L}$. However, there is no requirement that $\hat{L}$ be infinite or low. We say that $(\hat{D}, \hat{L})$ forces e $\notin G^{\prime}$ if there is no 
finite set $F$ which satisfies $(\hat{D}, \hat{L})$ with $e \in F^{\prime}$. Here satisfies is defined as for conditions, i.e., $\hat{D} \subseteq F \subseteq \hat{D} \cup \hat{L}$.

Define the predicate $P(Z)$ to hold if $Z \subseteq L,(D \cap A, Z)$ forces $e \notin G^{\prime}$, and $(D \cap \bar{A}, L-Z)$ forces $i \notin G^{\prime}$.

Note that $P$ is a $\Pi_{1}^{0, L}$-predicate. An index of $P$ as such a predicate may be computed effectively from a canonical index of the finite set $D \cap A$ and hence effectively from $0^{\prime}$ and a canonical index of $D$.

Case 1: $P(Z)$ holds for some $Z$.

By the Low Basis Theorem relative to $L$, there is a $Z$ such that $P(Z)$ holds and $Z \oplus L$ is low over $L$ and hence low. Fix such a $Z$. We may assume that we have a lowness index for $Z \oplus L$, by the effectiveness of the proof of the Low Basis Theorem and the fact that we are given a lowness index for $L$. Consider now the statements " $Z$ is infinite" and " $L-Z$ is infinite." Since $L$ is infinite, at least one of these statements is true. Further these are $\Pi_{2}^{0, L \oplus Z}$-statements and hence $\Pi_{2}^{0}$-statements, since $L \oplus Z$ is low, and $\Pi_{2}^{0}$-indices of the statements may be computed effectively from a lowness index of $L \oplus Z$, a canonical index of $D$, and an index of $P$ as a $\Pi_{1}^{0, L}$-predicate. Since $\mathbf{d}>>\mathbf{0}^{\prime}$, by Lemma 4.2 we can d-effectively select one of the statements which is true. If we select " $Z$ is infinite," let $\left(D^{*}, L^{*}\right)=(D, Z)$, which is clearly a condition. Then $e \notin(G \cap A)^{\prime}$ holds for all $G$ satisfying $\left(D^{*}, L^{*}\right)$ since convergent computations have a finite use. Similarly, if we select " $L-Z$ is infinite", let $\left(D^{*}, L^{*}\right)=(D, L-Z)$ and note that $k \notin(G \cap \bar{A})^{\prime}$ holds for all $G$ satisfying $\left(D^{*}, L^{*}\right)$.

Case 2: $P(Z)$ does not hold for any $Z$.

Then in particular $P(L \cap A)$ is false, and so there exists a finite set $F$ such that either

(1) $F$ satisfies $(D \cap A, L \cap A)$ and $e \in F^{\prime}$, or

(2) $F$ satisfies $(D \cap \bar{A}, L \cap \bar{A})$ and $i \in F^{\prime}$.

Search for such a finite set $F$. (This can be done effectively in $0^{\prime}$ since $A$ is $\Delta_{2}^{0}$.) For the first such $F$ which is found, let $u$ denote the use of the computation showing that $e \in F^{\prime}$ (if (i) applies), or $i \in F^{\prime}$ (if (ii) applies), and let $m$ be the least number which exceeds $u$ and all elements of $F$. Let $\left(D^{*}, F^{*}\right)=(D \cup F,\{z \in L: z>m\})$. If (i) holds and $G$ satisfies $(D, L)$, then $G \cap A$ satisfies $(D \cap A, L \cap A)$ so $e \in(G \cap A)^{\prime}$. Analogous comments (with $e$ replaced by $i$ and $A$ replaced by $\bar{A}$ ) apply if (ii) holds.

The completes the construction of $\left(D^{*}, L^{*}\right)$. Note that an index for $\left(D^{*}, L^{*}\right)$ can be found $\mathbf{d}-$-effectively since the distinction between Cases 1 and 2 is $L^{\prime}$-effective (and hence computable from $K$ ) and the action within each case can be carried out using $\mathbf{d}$ and $K$. 
The previous lemma is iterated as in the proof of Theorem 3.3 to produce a $\mathbf{d}$-effective sequence of conditions $\left(D_{0}, L_{0}\right),\left(D_{1}, L_{1}\right), \ldots$ such that $\left(D_{i+1}, L_{i+1}\right)$ extends $\left(D_{i}, L_{i}\right)$ for all $i$, and all sets $G$ satisfying $\left(D_{i}, L_{i}\right)$ also satisfy the requirement $R_{i}$. Let $G=\cup_{i} D_{i}$. Clearly $G \cap A$ and $G \cap \bar{A}$ are infinite. Further, for each pair $(e, k)$ the construction decides the value of $(G \cap A)^{\prime}(e)$ or of $(G \cap \bar{A})^{\prime}(k)$. It follows that the process either decides the value of $(G \cap A)^{\prime}(e)$ for all $e$, or it decides the value of $(G \cap \bar{A})^{\prime}(k)$ for all $k$. In the first case, $(G \cap A)^{\prime}$ has degree at most $\mathbf{d}$, and in the second case $(G \cap \bar{A})^{\prime}$ has degree at most $\mathbf{d}$.

\section{Constructing Low 2 Sets using Second Jump CONTRol}

We now present the proofs of Theorems 3.3 and 3.6 using direct control of the second jump. As explained in the introduction, it is these proofs which will be adapted to models of second order arithmetic to obtain our conservation results for Ramsey's theorem.

\subsection{Constructing a low 2 r-cohesive set using second jump con-} trol. We must construct a low 2 r-cohesive set $G$.

We build $G$ by forcing with conditions $(D, L)$ in which $D$ is a finite set and $L$ is an infinite low set such that every element of $D$ is less than every element of $L$. These are the same conditions used to prove Theorem 3.6 in Section 4.2 and the same definition of "extends" and "satisfies" applies here. Let $R_{0}, R_{1}, \ldots$ be a listing of all computable sets such that an index of the characteristic function of $R_{e}$ can be $0^{\prime \prime}$-effectively computed from $e$. Let $\sigma_{0}(G), \sigma_{1}(G), \ldots$ be an effective enumeration of all $\Sigma_{2}^{0}$ formulas having no free variable other than $G$.

The requirements are the following:

$$
\begin{gathered}
S_{3 e}:|G| \geq e \\
S_{3 e+1}: G \subseteq R_{e} \text { or } G \subseteq \overline{R_{e}} \\
S_{3 e+2}: \sigma_{e}(G) \text { is decided during the construction }
\end{gathered}
$$

As in Section 4 any sufficiently generic $G$ for these forcing conditions is $\mathrm{r}-$-cohesive. However, it would seem that these conditions are not appropriate for constructing a low 2 set since every sufficiently generic set $G$ is high, i.e., $0^{\prime \prime} \leq_{T} G^{\prime}$. To see this, note that for any condition $(D, L)$ and any computable function $f$ there is a condition $\left(D^{*}, L^{*}\right)$ extending $(D, L)$ such that any set $G$ which satisfies $\left(D^{*}, L^{*}\right)$ is such that $p_{G}$ (the principal function of $G$ ) dominates $f$. Hence any sufficiently generic set for these conditions dominates all computable functions and so is high. (As remarked in Seetapun and Slaman [1995, p. 580] the forcing conditions used by Seetapun to prove Theorem 2.7 also have high generic sets.) 
So how can such conditions be used to produce a low 2 set? In the proof of Theorem 3.3 in Section 4.1, the answer is that the construction must be $\mathbf{d}$-effective (where $\mathbf{d}$ is a given degree satisfying $\mathbf{d}>>\mathbf{0}^{\prime}$ ) and there is no reason to think that a condition $\left(D^{*}, L^{*}\right)$ forcing $p_{G}$ to dominate $f$ as above can be obtained $\mathbf{d}$-effectively. However, our current construction is only a $0^{\prime \prime}$-computable construction, and $\left(D^{*}, L^{*}\right)$ as above can be obtained $0^{\prime \prime}$-effectively. Thus, we cannot expect the analogue of Lemma 4.4 (replacing $\mathbf{d}$ by $\mathbf{0}^{\prime \prime}$ ) to hold. Instead we use a modified notion of forcing in which a condition not only involves a pair $(D, L)$ but also a "largeness" constraint. The generic sets will still be $r$-cohesive. However, the above argument that generic sets are high disappears because there is no "large" extension $\left(D^{*}, L^{*}\right)$ of $(D, L)$ forcing $p_{G}$ to dominate $f$. This will be explained in more detail later.

5.1.1. Deciding one $\Sigma_{2}^{0}$-formula $(\exists \vec{x}) \varphi(\vec{x}, G)$. We are given a condition $(D, L)$ and want to extend it in order to decide $(\exists \vec{x}) \varphi(\vec{x}, G)$ (possibly imposing a "largeness restriction" on all future conditions used in the construction).

Definition 5.1. Let $(D, L)$ be a condition.

(i) Let $\tau$ be a string and let $\theta(G)$ be a $\Delta_{0}^{0}$ formula. We say that $\tau$ forces $\theta(G)$ if the truth of $\theta(G)$ follows from $G$ extending $\tau$. More formally, this is defined by recursion in the standard manner. For example, a string $\tau$ forces the atomic formula $n \in G$ iff $\tau(n)=1$, and $\tau$ forces $n \notin G$ iff $\tau(n)=0$. The recursion then mirrors the definition of truth, except that negations are first "driven inwards" so that they apply only to atomic formulas.

(ii) $(D, L)$ forces a $\Pi_{1}^{0}$ formula $\varphi(G)$ if $\varphi(D \cup F)$ holds for all finite subsets $F$ of $L$.

(iii) Let the $\Pi_{1}^{0}$ formula $\varphi(G)$ be $(\forall \vec{x}) \theta(G, \vec{x})$, where $\theta(G, \vec{x})$ is a $\Delta_{0}^{0}$ formula. Then $(D, L)$ forces $\neg \varphi(G)$ if there is a tuple of $\vec{w}$ of numbers and a binary string $\tau$ such that $(D, L)$ extends $\tau$ and $\tau$ forces $\neg \theta(G, \vec{w})$. Here, to say that $(D, L)$ extends $\tau$ means that $\tau^{-1}(1) \subseteq D$ and $\tau^{-1}(0) \subseteq \overline{D \cup L}$.

We extend the above definition without change to pairs $(D, L)$ which are not necessarily conditions, as it does not require that $L$ be low or infinite. However, whenever we discuss pairs $(D, L)$, it will be the case that $D$ is finite and every element of $D$ is less than every element of $L$. It need not always be true that $L$ is infinite or low.

Note that if $(D, L)$ forces a $\Pi_{1}^{0}$ sentence $\varphi(G)$, then $\varphi(G)$ holds for all $G$ which satisfy $(D, L)$, since the failure of $\varphi(G)$ to hold uses only 
finitely much information about $G$. An analogous remark holds for forcing of negations of $\Pi_{1}^{0}$ formulas.

Let $(D, L)$ and $\left(D^{*}, L^{*}\right)$ be conditions. Say that $\left(D^{*}, L^{*}\right)$ is a finite extension of $(D, L)$ if $\left(D^{*}, L^{*}\right)$ extends $(D, L)$ and $L-L^{*}$ is finite. Note that if $(D, L)$ is a condition which does not force a $\Pi_{1}^{0}$ formula $\varphi(G)$, then there is a condition $\left(D^{*}, L^{*}\right)$ which is a finite extension of $(D, L)$ and forces $\neg \varphi(G)$.

We look for a finite partition of $L$ and a finite collection of finite extensions of $D$ in which each element of the partition forces some $\vec{w}$ to be a witness to $(\exists \vec{x}) \varphi(\vec{x}, G)$. That is, we look for sequences $\left(\vec{w}_{i}: i<n\right)$ and $\left(D_{i}, L_{i}: i<n\right)$ such that the $L_{i}$ are a partition of $L$; for each $i$, $D \subseteq D_{i} \subset D \cup L$; for each $i$, every element of $D_{i}$ is less than every element of $L_{i}$; and for each $i$, either $L_{i}$ has no element greater than $\max \left(\vec{w}_{i}\right)$ or $\left(D_{i}, L_{i}\right)$ forces $\varphi\left(\vec{w}_{i}, G\right)$. (There is no requirement that the $L_{i}$ 's be low or infinite.)

If such a collection exists, we can view a real $Z$ as representing such a collection, in which case the above clauses make a $\Pi_{1}^{0, L}$ property of $Z$ (fixing $n$, the set $\left(\vec{w}_{i}: i<n\right)$ and the finite sets $\left(D_{i}: i<n\right)$ beforehand).

Now, if there is a collection as above, then, by the Low Basis Theorem relative to $L$, there is $Z$ representing such a collection which is low relative to $L$ and hence low. Fix such a $Z$. Then all the $L_{i}$ 's it encodes are low and, since $L$ is infinite, at least one $L_{i}$ is infinite. It follows that for some $i<n,\left(D_{i}, L_{i}\right)$ is a condition extending $(D, L)$ and forcing $\varphi\left(\vec{w}_{i}, G\right)$. It is easily seen for such an $i$ that every set $G$ satisfying $\left(D_{i}, L_{i}\right)$ satisfies the formula $(\exists \vec{x}) \varphi(\vec{x}, G)$. If such a collection exists, we call the condition $(D, L)$ small, and otherwise we call $(D, L)$ large.

On the other hand, suppose that there is no such collection, so that $(D, L)$ is large. Then we have to ensure $(\forall \vec{x}) \neg \varphi(\vec{x}, G)$. To do this, we require that all conditions chosen in the remainder of the construction be large. Let $\left(D_{s}, L_{s}\right)$ be a large condition chosen at a future stage, and suppose at this stage we wish to ensure that $\neg \varphi(\vec{w}, G)$ holds for a particular tuple $\vec{w}$ by extending $\left(D_{s}, L_{s}\right)$. Since $\left(D_{s}, L_{s}\right)$ is large, it does not force $\varphi(\vec{w}, G)$. Then, as remarked after Definition 5.1, $\left(D_{s}, L_{s}\right)$ has a finite extension $\left(D^{*}, L^{*}\right)$ which forces $\neg \varphi(\vec{w}, G)$. It is easily seen that any finite extension of a large condition is large. Thus, by maintaining largeness and systematically considering all choices of $\vec{w}$ at future stages, we can ensure $(\forall \vec{x}) \neg \varphi(\vec{x}, G)$.

Now, we must also maintain largeness when we extend to meet the appropriate dense sets for r-cohesiveness. The requirements that $|G| \geq n$ are met using finite extensions and so largeness is preserved. 
Also if $R$ is a computable set then either $(D, L \cap R)$ or $(D, L \cap \bar{R})$ is a large condition. (If both of these are small, the partitions of $L$ witnessing the smallness of $(D, L \cap R)$ and $(D, L \cap \bar{R})$ could be combined in the obvious way to obtain a partition witnessing the smallness of $(D, L)$.) Furthermore, the construction can be carried out computably in $0^{\prime \prime}$. However, complications occur when we consider more than one $\Sigma_{2^{-}}^{0}$ formula.

5.1.2. Dealing with finitely many $\Sigma_{2}^{0}$-formulas. Suppose we implement the strategy of the preceding section considering successively all $\Sigma_{2}^{0}$ formulas. At any stage, we will have decided finitely many $\Sigma_{2}^{0}$ formulas. Suppose that at some stage we are committed to falsifying the $\Sigma_{2}^{0}$ formulas $\varphi_{1}(G), \varphi_{2}(G), \ldots, \varphi_{n}(G)$. Thus we are committed to falsifying $\varphi(G)$ where $\varphi(G)$ is a $\Sigma_{2}^{0}$ formula equivalent to $\varphi_{1}(G) \vee \varphi_{2}(G) \vee \cdots \vee \varphi_{n}(G)$. Thus we should commit ourselves to using forcing conditions which are large in the sense of the previous section for this $\varphi(G)$. This is basically what we do, although it is technically more convenient to work with the finite set $S=\left\{\varphi_{1}(G), \ldots, \varphi_{n}(G)\right\}$ than with the single formula $\varphi(G)$.

Definition 5.2. Let $(D, L)$ be a condition and let $S=\left\{\left(\exists \overrightarrow{x_{1}}\right) \varphi_{1}\left(\overrightarrow{x_{1}}, G\right)\right.$, $\left.\ldots,\left(\exists \overrightarrow{x_{k}}\right) \varphi_{k}\left(\overrightarrow{x_{k}}, G\right)\right\}$ be a finite set of $\Sigma_{2}^{0}$ formulas, with each formula $\varphi_{i}\left(\overrightarrow{x_{i}}, G\right)$ a $\Pi_{1}^{0}$ formula. We say that $(D, L)$ is $S$-small if there exist a number $n$ and sequences $\left(\vec{w}_{i}: i<n\right)$ and $\left(D_{i}, L_{i}, k_{i}: i<n\right)$ such that the $L_{i}$ 's are a partition of $L$; for each $i, D \subseteq D_{i} \subset D \cup L$; for each $i$, every element of $D_{i}$ is less than every element of $L_{i}$; and for each $i$, either $L_{i}$ has no element greater than $\max \left(\vec{w}_{i}\right)$ or $\left(D_{i}, L_{i}\right)$ forces $\varphi_{k_{i}}\left(\vec{w}_{i}, G\right)$. (There is no requirement that the $L_{i}$ 's be low or infinite.) Otherwise, $(D, L)$ is called $S$-large. (It is easily seen that this definition is independent of the indexing of $S$.)

Assume $(D, L)$ is $S$-large. If $S^{*} \subseteq S$ then $(D, L)$ is $S^{*}$-large. If $\left(D^{*}, L^{*}\right)$ is a finite extension of $(D, L)$, then $\left(D^{*}, L^{*}\right)$ is also $S$-large. Also, note that if $(D, L)$ is $S$-large, and $\left(D_{1}, L_{1}\right), \ldots,\left(D_{n}, L_{n}\right)$ are extensions of $(D, L)$ with $\cup_{i=1}^{n} L_{i}=L$, then $\left(D_{i}, L_{i}\right)$ is $S$-large for some $i \leq n$.

If $\theta(\vec{x}, G)$ is a $\Pi_{1}^{0}$-formula, then for each tuple $\vec{w}$ of constants of the same length as $\vec{x}$, the formula $\theta(\vec{w}, G)$ is called a $\Pi_{1}^{0}$ instance of the $\Sigma_{2}^{0}$ formula $(\exists \vec{x}) \theta(\vec{x}, G)$.

Suppose that at some stage of the construction of $G$ we have committed ourselves to ensuring the falsity of the formulas in $S$, where $S$ is a finite set of $\Sigma_{2}^{0}$ formulas with at most $G$ free, and let $\varphi(G)$ be a $\Sigma_{2}^{0}$ 
formula we now wish to decide. Let $(D, L)$ be the condition we are considering at this stage, so that $(D, L)$ is $S$-large and we are committed to working with $S$-large conditions in the future. Now we ask whether $(D, L)$ is $(S \cup\{\varphi(G)\})$-large. If it is, we commit ourselves to ensuring that $\varphi(G)$ is false and to working only with $(S \cup\{\varphi(G)\})$-large conditions at all future stages. The next lemma will then show that all $\Pi_{1}^{0}$ instances of $\varphi(G)$ can be falsified at future stages. On the other hand, if $(D, L)$ is $(S \cup\{\varphi(G)\})$-small, Lemma 5.5 will show that $(D, L)$ has an $S$-large extension which forces some $\Pi_{1}^{0}$ instance of $\varphi(G)$.

An index of a condition is given by a pair $(a, b)$, where $a$ is a canonical index of $D$ and $b$ is a lowness index of $L$, i.e., $L^{\prime}=\{b\}^{K}$.

Lemma 5.3. Suppose that $S$ is a finite set of $\Sigma_{2}^{0}$ formulas with no free variable other than $G,(D, L)$ is an $S$-large condition, and $\psi(G)$ is a $\Pi_{1}^{0}$ instance of some formula in $S$. Then $(D, L)$ has an $S$-large extension $\left(D^{*}, L^{*}\right)$ which forces $\neg \psi(G)$. Furthermore, an index of $\left(D^{*}, L^{*}\right)$ can be computed using an oracle for $0^{\prime}$ from an index of $(D, L)$, the canonical index of $S$, and the Gödel number of $\varphi(G)$.

Proof. Since $(D, L)$ is $S$-large, it does not force $\psi(G)$. Hence, $(D, L)$ has a finite extension $\left(D^{*}, L^{*}\right)$ which forces $\neg \psi(G) .\left(D^{*}, L^{*}\right)$ is $S$-large because it is a finite extension of the $S$-large condition $(D, L)$. To find such a $\left(D^{*}, L^{*}\right)$, search for finite sets $F_{0}, F_{1}$ such that $D \subseteq F_{0} \cup L \cup D$ and $\left(F_{0}, L-F_{1}\right)$ forces $\neg \varphi(G)$. This is a $0^{\prime}$-effective search and must terminate by the argument above. Let $\left(D^{*}, L^{*}\right)=\left(F_{0}, L-F_{1}\right)$.

Lemma 5.4. There is a $0^{\prime \prime}$-effective procedure to decide, given an index of a condition $(D, L)$ and a canonical index of a finite set $S$ of $\Sigma_{2}^{0}$ formulas, whether $(D, L)$ is $S$-large. Furthermore, if $(D, L)$ is $S-$ small, there are low sets $L_{i}$ which witness this, and one may compute from a $0^{\prime}$-oracle a value of $n$, lowness indices for $\left(L_{i}: i<n\right)$ and also the corresponding sequences $\left(\vec{w}_{i}: i<n\right)$ and $\left(D_{i}, L_{i}, k_{i}: i<n\right)$ which witness that $(D, L)$ is $S$-small as in Definition 5.2.

Proof. The definition of $S$-smallness of $(D, L)$ can be put in the form $(\exists z)(\exists Z) P(z, Z, D, L, S)$, where $P$ is a $\Pi_{1}^{0}$ predicate. (Here $z$ codes the number $n$ and the sequences $\left(\vec{w}_{i}: i<n\right)$ and $\left(D_{i}, k_{i}: i<n\right)$ from the definition of smallness and $Z$ codes $\left(L_{i}: i<n\right)$.) Then the predicate $(\exists Z) P(z, Z, D, L, S)$ is a $\Pi_{1}^{0, L}$ predicate, as it asserts that a certain $L$-computable tree in $2^{<\omega}$ of known index contains strings of every length. From a lowness index of $L$ one may find a $\Delta_{2}^{0}$ index of the same predicate as a predicate of $z, D$, and $S$, and hence a $\Sigma_{2}^{0}$ index of $(\exists z)(\exists Z) P(z, Z, D, L, S)$. Thus there is a $\Sigma_{2}^{0}$ formula $\lambda(a, b)$ such that, whenever $a$ is an index of a condition $(D, L)$ and $b$ is the canonical 
index of a finite set of $\Sigma_{2}^{0}$ formulas with at most $G$ free, $(D, L)$ is $S$ small iff $\lambda(a, b)$ holds. (Note that we assert nothing about the truth value of $\lambda(a, b)$ when $a$ is not an index of a condition.) Assume now that $(D, L)$ is $S$-small. Then we may search effectively relative to $0^{\prime}$ for a $z$ such that $(\exists Z) P(z, Z, D, L, S)$ holds. Fixing such a $z$, by the Low Basis Theorem relative to $L$, there is a $Z$ such that $P(z, Z, D, L, S)$ holds and $Z$ is low relative to $L$ and hence low. By the uniformity of the proof of the Low Basis theorem, a lowness index of $Z$ may be found effectively from a lowness index of $L$. Lowness indices of the $L_{i}$ 's may be effectively computed from a lowness index of $Z$.

Lemma 5.5. Assume that $(D, L)$ is $S$-large and $(S \cup\{\gamma(G)\})$-small, where the formula $\gamma(G)$ is $\Sigma_{2}^{0}$. Then there exists an $S$-large condition $\left(D^{*}, L^{*}\right)$ extending $(D, L)$ which forces $\gamma(G)$. Furthermore one can find an index for $\left(D^{*}, L^{*}\right)$ by applying a $0^{\prime \prime}$-computable function to an index of $(D, L)$, a canonical index of $S$, and a Gödel number of $\gamma(G)$.

Proof. By the previous lemma, we may choose the sets $L_{i}$ which witness that $(D, L)$ is $S \cup\left\{(\exists \vec{x}) \varphi_{k}(\vec{x}, G)\right\}$-small to be low over $L$ and hence low. Fix corresponding $n, D_{i}$ for $i<n$ and $\Pi_{1}^{0}$ instances of formulas in $S \cup\{\gamma(G)\}$.

Let's restrict our attention to those $i$ where $\left(D_{i}, L_{i}\right)$ forces some $\Pi_{1}^{0}$ instance of $\gamma(G)$. Since $(D, L)$ is $S$-large, at least one of these $\left(D_{i}, L_{i}\right)$ must be $S$-large (otherwise $(D, L)$ would be $S$-small), and hence may serve as our desired $\left(D^{*}, L^{*}\right)$. By Lemma 5.4 we may find such an $i$ computably in $0^{\prime \prime}$.

Let $R$ be a computable set and let $(D, L)$ be an $S$-large condition. Then at least one of $(D, L \cap R)$ or $(D, L \cap \bar{R})$ is $S$-large (since otherwise $(D, L)$ would be $S$-small). Using an oracle for $0^{\prime \prime}$ we can identify one of these which is $S$-large. Hence we can satisfy the r-cohesiveness requirements without violating our commitment to work with $S$-large conditions. Similarly, we can meet the requirements $|G| \geq k$ by finite extensions which, as has been noted, preserve $S$-largeness.

(The definition of smallness and the lemmas following the definition are key to some of our proofs. There will be several additions to this definition throughout the paper. Each time we add to the definition we must verify that the appropriate versions of the above lemmas hold.)

5.1.3. Putting it all together. This is a standard $0^{\prime \prime}$-computable forcing construction. However, the conditions should be thought of as triples $(D, L, S)$ such that $(D, L)$ is an $S$-large condition as defined above. We say that $\left(D^{*}, L^{*}, S^{*}\right)$ extends $(D, L, S)$ if $\left(D^{*}, L^{*}\right)$ extends $(D, L)$ and $S^{*} \supseteq S$. Lemmas 5.35 .5 show that an appropriately generic 
$0^{\prime \prime}$-computable set for this forcing is $\mathrm{r}-$ cohesive and $\mathrm{low}_{2}$. For completeness, the details are spelled out below.

We will work computably in $0^{\prime \prime}$. Let $\left\{R_{i}\right\}$ be a listing of all computable sets such that an index of $R_{i}$ as a computable set can be computed from $i$ effectively relative to $0^{\prime \prime}$. Let $(\exists \vec{x}) \varphi_{0}(\vec{x}, G),(\exists \vec{x}) \varphi_{1}(\vec{x}, G), \ldots$ be a computable listing of all $\Sigma_{2}^{0}$-formulas with at most $G$ free. (The $\varphi_{i}$ 's are $\left.\Pi_{1}^{0}\right)$. Let $\theta_{0}(G), \theta_{1}(G), \ldots$ be a computable listing of all $\Pi_{1}^{0}$ formulas with at most $G$ free such that each such formula occurs infinitely often in the list.

Computably in $0^{\prime \prime}$, we will construct conditions $\left(D_{s}, L_{s}\right)$ and indices for these conditions such that $G=\cup_{s \in \mathbb{N}}\left\{D_{s}\right\}$ is r-cohesive and low ${ }_{2}$. In addition, we construct finite sets $\left\{S_{s}\right\}$ of $\Sigma_{2}^{0}$-formulas with $S_{s} \subseteq S_{s+1}$. During and after stage $s$, we will commit ourselves to working with $S_{s^{-}}$ large conditions and ensuring $(\forall \vec{x}) \neg \varphi_{j}(\vec{x}, G)$ for all $(\exists \vec{x}) \varphi_{j}(\vec{x}, G) \in S_{s}$. Initially, let $\left(D_{-1}, L_{-1}\right)=(\emptyset, \mathbb{N}),\left(a_{-1}, b_{-1}\right)$ be an index for $\left(D_{-1}, L_{-1}\right)$ and $S_{-1}=\emptyset$. We can assume inductively that $\left(D_{s-1}, L_{s-1}\right)$ is $S_{s-1^{-}}$ large.

Stage s: If $\left(D_{s-1}, L_{s-1}\right)$ is $\left(S_{s-1} \cup\left\{(\exists \vec{x}) \varphi_{s}(\vec{x}, G)\right\}\right)$-small then let $S_{s}=S_{s-1}$ and as in Lemma 5.5 extend to a $S_{s}$-large condition $\left(D_{s}^{*}, L_{s}^{*}\right)$ which forces $\varphi_{s}(\vec{w}, G)$, for some $\vec{w}$. (As we noted in Lemma 5.5 an index for $\left(D_{s}^{*}, L_{s}^{*}\right)$ can be found effectively in $\mathbf{0}^{\prime \prime}$.) Otherwise let $S_{s}=S_{s-1} \cup\left\{(\exists \vec{x}) \varphi_{s}(\vec{x}, G)\right\}$ and $\left(D_{s}^{*}, L_{s}^{*}\right)=\left(D_{s-1}, L_{s-1}\right)$. (By Lemma 5.4, determining which of these cases applies can be done effectively in $0^{\prime \prime}$.) Next, if $\theta_{s}(G)$ is a $\Pi_{1}^{0}$ instance of some formula in $S_{s}$, let $\left(D^{* *}, L^{* *}\right)$ be an $S_{s}$-large extension of $\left(D^{*}, L^{*}\right)$ which forces $\theta_{s}(G)$. Such a condition exists by Lemma 5.3, and an index of it may be found effectively from $0^{\prime \prime}$ by the same lemma. Furthermore, it is easy to arrange that $\left|D^{* *}\right| \geq s$ by taking a finite extension if necessary. Finally, if $L^{* *} \cap R_{s}$ is infinite, let $\left(D_{s}, L_{s}\right)=\left(D^{* *}, L^{* *} \cap R_{s}\right)$, and otherwise let $\left(D_{s}, L_{s}\right)=\left(D^{* *}, L^{* *} \cap \bar{R}\right)$.

The parenthetical remarks in the above construction show that we can effectively find the indices for all the constructed conditions as we proceed and that the construction can be done computably in $\mathbf{0}^{\prime \prime}$. Clearly $G$ is $\mathrm{r}$-cohesive. Since we decide computably in $\mathbf{0}^{\prime \prime}$ all $\Sigma_{2}^{0}$ formulas relative to $G, G$ is $\operatorname{low}_{2}$.

5.2. Proving Theorem 3.6 by controlling the double jump. We will assume the reader is familiar with the argument presented in Section 5.1 and will argue in a similar vein. Let $A$ be a $\Delta_{2}^{0}$ set. For ease of notation in the next subsection, we will let $A_{0}=A$ and $A_{1}=\bar{A}$. We assume without loss of generality that for all $i, A_{i}$ does not have an 
infinite low subset. We will build an infinite set $G$ such that for some $i, G \subseteq A_{i}$ and $G$ is $\operatorname{low}_{2}$.

It would be pleasant if we could completely adopt the argument in Section 5.1 but there are some major problems. Previously, our concern was to make $G \mathrm{r}-$ cohesive and $\operatorname{low}_{2}$; here our concern is to make an infinite low $_{2}$ set $G$ contained in $A_{0}$ or $A_{1}$. (We need not and will not make $G$ be r-cohesive also, although this feature could easily be added to the argument.)

As a first approximation, let's attempt to build an infinite low $_{2}$ set $G \subseteq A_{0}$ by modifying the method of Section 5.1. Hence we will only work with conditions $(D, L)$ where $D \subset A_{0}$.

In this case, we must modify Definition 5.2 of $S$-small by requiring, in addition, for each $i, D_{i} \subset A_{0}$; we will call this modified definition $S$ -

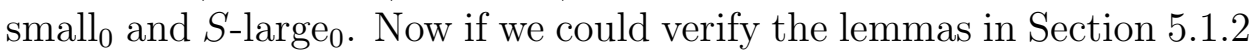
for this modified definition of smallness we would be done.

The first (and only) place we get into trouble is the following: There may exist a condition $(D, L)$ and a finite set $S$ of $\Sigma_{2}^{0}$ formulas such that $(D, L)$ is $S$-large but $\left(D, L \cap A_{0}\right)$ is $S$-small - $_{\text {. }}$ (Note that $L \cap A_{0}$ need not be low, so $\left(D, L \cap A_{0}\right)$ need not be a condition, but the definition

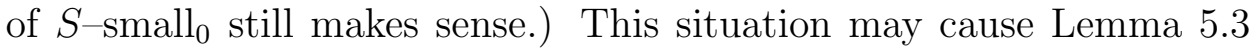
to break down. Large $e_{0}$-ness only implies the existence of a $D^{*}$ not a $D^{*}$ such that $D^{*} \subset A_{0}$. Thus, we may commit ourselves to falsifying a $\Sigma_{2}^{0}$ formula $\varphi(G)$ and later find that there is a $\Pi_{1}^{0}$ instance of $\varphi(G)$ that we are unable to satisfy by adding elements of $A_{0}$ to $G$. In this situation, we try to build an infinite low $_{2}$ set $G \subseteq A_{1}$.

5.2.1. No Problem. We did not have a problem modifying the arguments in Section 5.1 .2 if for all conditions $(D, L)$ and all finite sets $S$ of $\Sigma_{2}^{0}$ formulas with at most $G$ free, $(D, L)$ is $S$-large implies $_{0}\left(D, L \cap A_{0}\right)$

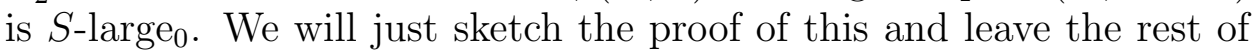
the verification of this to the reader. With the above assumption the proof of the modified Lemma 5.3 goes almost the same with large ${ }_{0}$ ness replacing largeness. As for Lemma 5.4 a condition $(D, L)$ being $S$ small $_{0}$ can be stated as $(\exists z)\left[R^{A}(z) \&(\exists Z) P(z, Z, D, L, S)\right]$, where $R^{A}$ is an $A$-computable predicate. For fixed $L$ and $S$, the matrix of this is $\Delta_{2}^{0}$, so this is a $\Sigma_{2}^{0}$ predicate whose index can be effectively computed from an index of the condition $(D, L)$. This is the only situation where we use the hypothesis that $A$ is $\Delta_{2}^{0}$, instead of merely $\Delta_{3}^{0}$. So, in fact, it is enough that $A$ be low over $0^{\prime}$. The proof of the modified version of Lemma 5.5 is the same as the proof of Lemma 5.5. To ensure that $G$ is infinite we observe that for any condition $(D, L)$ with $D \subseteq A_{0}$ and any $k$, there is a finite extension $\left(D^{*}, L^{*}\right)$ of $(D, L)$ with $D^{*} \subseteq A_{0}$ 
and $\left|D^{*}\right| \geq k$. This follows easily from our assumption that $A_{1}$ has no infinite low subset, so that $L \cap A_{0}$ is infinite.

5.2.2. Handling the problem. Hence we may assume that there is a condition $(D, L)$ and a finite set of $\Sigma_{2}^{0}$-formulas such that $D \subseteq A_{0}$,

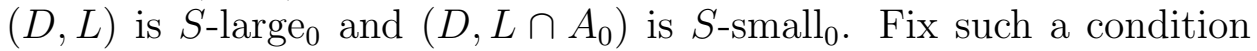
$(\tilde{D}, \tilde{L})$ and such a set $\tilde{S}$.

We now try to construct an infinite low 2 set $G \subseteq A_{1}$. However, if we try to do this by simply replacing $A_{0}$ by $A_{1}$ in the argument of the previous section, we may run into the same problem. Instead, we take advantage of our failure on the $A_{0}$-side to ensure success on the $A_{1}-$ side. We will need a slightly different notion of smallness and largeness. But once we have such a definition the proof will go almost through as before. However to be safe we will provide the details.

We will restrict ourselves to conditions $(D, L)$ where $D \subseteq A_{1}, L \subseteq \tilde{L}$, and $(\tilde{D}, L)$ is $\tilde{S}$-large $_{0}$. We call such conditions 1 -acceptable. Note that $(\emptyset, \tilde{L})$ is 1 -acceptable. This condition will be used as the initial condition in the construction of $G$.

Definition $5.6\left(\mathrm{Small}_{1}\right.$ and Large $)$. Let $(D, L)$ be a 1 -acceptable condition, and let $S$ be a finite set of $\Sigma_{2}^{0}$ formulas with at most $G$ free. Then $(D, L)$ is $S$-small 1 if there exist $n$, a partition $\left(L_{i}: i<n\right)$ of $L$ and finite sets $\left(D_{i}: i<n\right)$ such that for each $i<n, D_{i} \subseteq A_{1} \cap(D \cup L)$, every element of $D_{i}$ is less than every element of $L_{i}$, and either $L_{i}$ is finite, or $\left(D_{i}, L_{i}\right)$ forces a $\Pi_{1}^{0}$ instance of some formula in $S$, or $\left(\tilde{D}, L_{i}\right)$ forces a $\Pi_{1}^{0}$ instance of some formula in $\tilde{S}$. If $(D, L)$ is not $S$-small, then it is $S$-large . $_{\text {. }}$

This definition is highly dependent on $\tilde{D}, \tilde{L}$ and $\tilde{S}$. Assume $(D, L)$ is $S$-large - If $^{*} \subseteq S$ then $(D, L)$ is $S^{*}$-large $_{1}$. If $\left(D^{*}, L^{*}\right)$ is a 1acceptable finite extension of a 1 -acceptable $S$-large - condition $_{1}(D, L)$, then $\left(D^{*}, L^{*}\right)$ is also $S$-large $_{1}$. It is enough to prove modified versions of Lemmas 5.3, 5.4 and 5.5. But first we need the following lemma to show that the difficulty we had on the $A_{0}$ side will not arise again on the $A_{1}$ side.

Lemma 5.7. If $(D, L)$ is $S$-large $_{1}$ then $\left(D, L \cap A_{1}\right)$ is $S$-large . $_{1}$

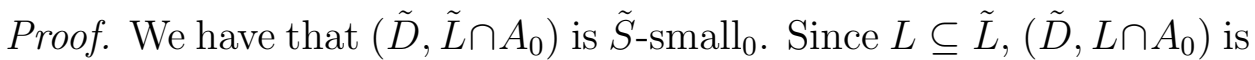

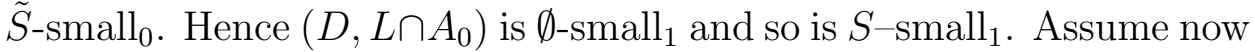
that $\left(D, L \cap A_{1}\right)$ is also $S$-small s $_{1}$ Then the witnesses for $S$-small ${ }_{1}$ ness of $\left(D, L \cap A_{0}\right)$ and $\left(D, L \cap A_{1}\right)$ could be combined to show that $(D, L)$ is $S$-small, which is the desired contradiction. 
Lemma 5.8. Suppose that $(D, L)$ is an $S$-large $_{1}$ condition and that $\theta(G)$ is a $\Pi_{1}^{0}$ instance of some formula in $S$. Then there is a 1acceptable extension $\left(D^{*}, L^{*}\right)$ of $(D, L)$ such that $\left(D^{*}, L^{*}\right)$ is $S$-large 1 and forces $\neg \theta(G)$. Furthermore given an index for $(D, L)$ and a Gödel number of $\theta(G)$, an index for $\left(D^{*}, L^{*}\right)$ can be found effectively in $0^{\prime}$.

Proof. Since $(D, L)$ is $S$-large , $_{1}$ it follows from Lemma 5.7 that $\left(D, L \cap A_{1}\right)$ is $S$-large , so that $\left(D, L \cap A_{1}\right)$ does not force $\theta(G)$. It follows that $\left(D, L \cap A_{1}\right)$ has a finite extension $\left(D^{*}, L^{*}\right)$ which forces $\neg \theta(G)$. Then $\left(D^{*}, L^{*}\right)$ is easily seen to be 1 -acceptable, and it is

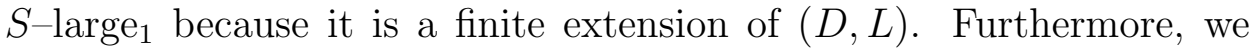
can find it by an $A_{1} \oplus L^{\prime}$-effective search, and $A_{1} \oplus L^{\prime} \leq_{T} 0^{\prime}$.

Lemma 5.9. There is a $0^{\prime \prime}$-effective procedure to decide, given an index of a condition $(D, L)$ and a canonical index of a finite set $S$ of

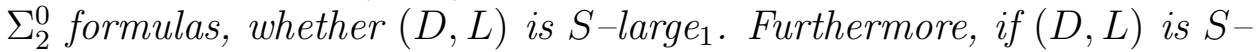
small ${ }_{1}$, there are low sets $L_{i}$ which witness this, and one may compute from a $0^{\prime}$-oracle a value of $n$, lowness indices for $\left(L_{i}: i<n\right)$ and also the corresponding sequences $\left(\vec{w}_{i}: i<n\right)$ and $\left(D_{i}, L_{i}, k_{i}: i<n\right)$ which witness that $(D, L)$ is $S-$ small $_{1}$ as in Definition 5.2.

This lemma is proved by virtually the same argument as Lemma 5.4.

Lemma 5.10. Assume that $(D, L)$ is $\left(S \cup\left\{(\exists \vec{x}) \varphi_{k}(\vec{x}, G)\right\}\right)$-small s $_{1}$. Then there exists a condition $\left(D^{*}, L^{*}\right)$ which is $S$-large ${ }_{1}$ and forces $\varphi_{k}(\vec{w}, G)$, for some $\vec{w}$. Furthermore given an index for $(D, L)$ one can find computably in $L^{\prime \prime} \equiv_{T} \mathbf{0}^{\prime \prime}$ an index for $\left(D^{*}, L^{*}\right)$.

Proof. Same as the proof of Lemma 5.5.

5.2.3. Putting it all together. The construction of an infinite $\operatorname{low}_{2}$ set $G$ contained in $A_{0}$ or $A_{1}$ is closely parallel to that in Section 5.1. If there do not exist a finite set $S$ of $\Sigma_{2}^{0}$ formulas and an $S$-large condition $(D, L)$ such that $\left(D, L \cap A_{0}\right)$ is $S$-large, we iterate the lemmas mentioned in Section 5.2.1 to construct a low 2 set $G \subseteq A_{0}$. Otherwise, by Lemmas $5 . 8 \longdiv { 5 . 1 0 }$ in Section 5.2.2, we can ensure that $G \subseteq A_{1}$. We omit these routine details.

\subsection{A proof of Theorem 3.7 by controlling the double jump.}

As we noted shortly after the statement of Theorem 3.7, Theorem 3.7 follows from Theorem 3.6. So this section is unnecessary from the point of view of computability theory. However, our proofs of Theorems 10.4 and 11.2 will be based on adapting this proof to models of arithmetic.

We will assume the reader is very familiar with the argument presented in Section 5.2 and will argue in a similar vein. Let $A_{i}$ be $k$ many 
$\Delta_{2}^{0}$ sets such that $\sqcup_{i<k} A_{i}=\mathbb{N}$. We will build an infinite low lot $_{2}$ set such that for some $i, A_{i} \subseteq G$.

As in Section 5.2, we will first try to make $G$ a subset of $A_{0}$, if that fails we will try to make $G$ a subset of $A_{1}$, and if that fails we will try to make $G$ a subset of $A_{2}$ and so on. But in Section 5.2 in order to make $G$ a subset of $A_{1}$ we need the witness to the reason we failed to make $G$ a subset of $A_{0}$ in order to successfully make $G$ a subset of $A_{1}$. We will use the function $W$ to witness these failures. Hence our definition of smallness and largeness will depend on $W$.

We consider $W$ as a possibly empty finite function. The domain of $W$ will be some finite initial segment of $\mathbb{N}$. Let $|W|$ (the length of $W$ ) be the least number not in the domain of $W$. The values of $W$ are triples $(D, L, S)$ such that $(D, L)$ is a condition and $S$ is a finite set of $\Sigma_{2}^{0}$ formulas with at most $G$ free. If $i$ is in the domain of $W$, then we denote $W(i)$ by $\left(\tilde{D}_{W(i)}, \tilde{L}_{W(i)}, \tilde{S}_{W(i)}\right)$.

Definition 5.11 $\left(\right.$ Small $_{W}$ and $\left.\operatorname{Large}_{W}\right)$. Let $W$ be a finite partial function with $|W|<k$ as we have described. Let $l=|W|-1$. We will restrict ourselves to conditions $(D, L)$ where $D \subseteq A_{|W|}$ and $L \subseteq \cap_{j \leq l} \tilde{L}_{W}(j)$. We call such conditions $W$-acceptable. Let $(D, L)$ be $W$-acceptable condition and let $S$ be a finite set of $\Sigma_{2}^{0}$ formulas with at most $G$ free.

Then $(D, L)$ is $S-$ small $_{W}$ if there exist $n$ and a partition $\left(L_{i}: i<n\right)$ of $L$ and finite sets $\left(D_{i}: i<n\right)$ such that, for each $i<n$, every element of $D_{i}$ is less than every element of $L_{i}, L_{0}, L_{1}, \ldots, L_{n-1}$ is a partition of $L$, and, for each $i<n$, either $D_{i} \subseteq A_{|W|} \cap(D \cup L)$ and $\left(D_{i}, L_{i}\right)$ forces a $\Pi_{1}^{0}$ instance of some formula in $S$, or there exists $j<|W|$ such that $\left(\tilde{D}_{W(j)}, L_{i}\right)$ forces a $\Pi_{1}^{0}$ instance of some formula in $\tilde{S}_{W(j)}$, or $L_{i}$ is finite. If $(D, L)$ is not $S$-small $W$, it is called $S$-large ${ }_{W}$.

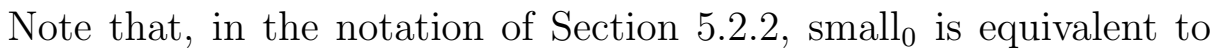
small $_{\emptyset}$, large $e_{0}$ is equivalent to large $\emptyset$, small $_{1}$ is equivalent to small $_{W}$, and large $_{1}$ is equivalent to large $_{W}$, where $|W|=1$ and $W(0)=(\tilde{D}, \tilde{L}, \tilde{S})$.

Lemma 5.12. There is a $W$ such that $|W|<k$ and for all conditions $(D, L)$ with $D \subseteq A_{|W|}$ and $L \subseteq \cap_{i<|W|} \tilde{L}_{i}$ and for all finite sets $S$ of $\Sigma_{2}^{0}$ formulas, if $(D, L)$ is $S$-large ${ }_{W}$ then $\left(D, L \cap A_{|W|}\right)$ is $S$-large ${ }_{W}$. (Recall that $k$ is the number of $\Delta_{2}^{0}$ sets we have partitioned $\mathbb{N}$ into.) Furthermore, $\left(\emptyset, \cap_{i<|W|} \tilde{L}_{i}\right)$ is a condition which is $\emptyset$-large ${ }_{W}$.

Proof. We define $W$ inductively. Assume that $W \uparrow i$ is defined. If $i=k$, stop the induction and set $W=W \uparrow i$. Ask whether there is a $W \uparrow i$-acceptable condition $(\tilde{D}, \tilde{L})$ and a set $\tilde{S}$ such that $(\tilde{D}, \tilde{L})$ is 
$\tilde{S}$-large $_{W\lceil i}$ and $\left(\tilde{D}, \tilde{L} \cap A_{i}\right)$ is $\tilde{S}$-small $_{W\lceil i}$. If not, we let $W=W\lceil i$ and end the induction. Otherwise let $\tilde{D}_{W(i)}=\tilde{D}, \tilde{L}_{W(i)}=\tilde{L}$ and $\tilde{S}_{W(i)}=\tilde{S}$ and continue the induction.

Suppose temporarily that $|W|<k$. Then by the definition of $W$, it is the case that for all $W$-acceptable conditions $(D, L)$ and for all finite sets $S$ of $\Sigma_{2}^{0}$ formulas if $(D, L)$ is $S$ - $\operatorname{large}_{W}$, then $\left(D, L \cap A_{|W|}\right)$ is $S$-large ${ }_{W}$.

It remains to show that $|W|<k$. Assume otherwise; $|W| \geq k$. Let $l=k-1$ and $n<l$. Let $D=\tilde{D}_{W(l)}, L=\tilde{L}_{W(l)}, S=\tilde{S}_{W(l)}$.

By the inductive definition of $W$, we have that $\left(\tilde{D}_{W(n)}, \tilde{L}_{W(n)} \cap A_{n}\right)$ is $\tilde{S}_{W(n)}$-small ${ }_{W\lceil n}$. Inductively assume that $\left(D, L \cap\left(\sqcup_{i<n} A_{i}\right)\right)$ is $\emptyset$-small $W \backslash n$. Since $L \subseteq \tilde{L}_{W(n)}$ (as $(D, L)$ is $W\lceil l$-acceptable), $\left(\tilde{D}_{W(n)}, L \cap A_{n}\right)$ is $\tilde{S}_{W(n)}$-small $_{W\lceil n} . \quad$ So $\left(\tilde{D}_{W(n)}, L \cap\left(\sqcup_{i<n+1} A_{i}\right)\right)$ is $\tilde{S}_{W(n)}$-small ${ }_{W\lceil n}$. Hence $\left(D, L \cap\left(\sqcup_{i<n+1} A_{i}\right)\right)$ is $\emptyset$-small $W \backslash(n+1)$. Therefore $\left(D, L \cap\left(\sqcup_{i<l} A_{i}\right)\right)$ is $\emptyset$-small sm $_{W l}$.

Therefore if $\left(D, L \cap A_{l}\right)$ is $S$-small s $_{W l}$ then $(D, L)$ must be $S$-small $W\lceil$. This contradicts the choice of $D, L$ and $S$.

It remains to be shown that $\left(\emptyset, \cap_{i<|W|} \tilde{L}_{i}\right)$ is a condition which is $\emptyset-$ $\operatorname{large}_{W}$. This is clear if $|W|=0$, since then $\cap_{i<|W|} \tilde{L}_{i}=\mathbb{N}$ by convention. If $|W|=j>0$, then $W(j-1)$ is chosen so that $\tilde{D}_{W(j-1)}, \tilde{L}_{j-1}$ is $\tilde{S}_{j-1^{-}}$ large. From this it follows easily that $\left(\emptyset, \cap_{i<|W|} \tilde{L}_{i}\right)$ is a condition which is $\emptyset-\operatorname{large}{ }_{W}$.

Fix such a $W$. We can now prove the modified versions of Lemmas 5.8, 5.9 and 5.10. The proofs of these modified lemmas are essentially the same as the proofs of Lemmas 5.8, 5.9 and 5.10 which are found in Section 5.2.2. With these modified lemmas in hand the construction of $G$ proceeds as in Section 5.2.3. In particular, all conditions used in the construction are $\emptyset$-large ${ }_{W}$ conditions, where $W$ is chosen to satisfy Lemma 5.12, and the construction produces an infinite $\mathrm{low}_{2}$ set $G \subseteq A_{|W|}$. The initial condition is $\left(\emptyset, \cap_{i<|W|} \tilde{L}_{i}\right)$, which is $\emptyset$-large by Lemma 5.12 .

\section{SeCond order ARIthmetic AND CONSERVATion}

Here we present some basic information on second order arithmetic and conservation theorems. For further information, see, for example, Simpson [1999].

The language of second order arithmetic is a sorted language with the symbols: $=, \in,+, \times, 0,1,<$ (the usual symbols of arithmetic with the additional symbol $\in$ ); number variables: $n, m, x, y, z \ldots$ (always lower case letters); and set variables: $X, Y, Z, \ldots$ (always capital letters). 
First order terms are built up in the usual way (without using set variables). Atomic formulas are those of the form $t=u, t<u$, and $t \in X$, where $t$ and $u$ are first order terms. Formulas are then built up as usual. A model in this language is of the form $(\mathbb{X}, \mathcal{F},+, \times, 0,1,<)$, where $\mathcal{F} \subseteq \mathcal{P}(\mathbb{X})$. The elements of $\mathbb{X}$ are sometimes called the numbers of $\mathcal{M}$ and the elements of $\mathcal{F}$ the reals of $\mathcal{M}$. In interpreting truth of a formula in this model, the number variables range over the numbers of $\mathcal{M}$ and the set variables range over the reals of $\mathcal{M}$. The intended model of second order arithmetic is $(\mathbb{N}, \mathcal{P}(\mathbb{N}),+, \times, 0,1,<)$.

\section{Definition 6.1.}

(i) A first order formula is a formula without any set variables.

(ii) An arithmetic formula is a formula without any quantification over set variables (although free set variables may occur).

(iii) $\varphi$ is $\Delta_{0}^{0}$ if $\varphi$ is an arithmetic formula with all quantifiers bounded

(iv) If $\varphi(\vec{x})$ is $\Delta_{0}^{0}$ then $(\exists \vec{x})[\varphi(\vec{x})]$ is $\Sigma_{1}^{0}$ and $(\forall \vec{x})[\varphi(\vec{x})]$ is $\Pi_{1}^{0}$. (So, for example, $(\exists x)(\forall y<x)[x \times y \in X]$ is $\Sigma_{1}^{0}$.)

(v) If $\varphi(\vec{x})$ is $\Pi_{n}^{0}$ then $(\exists \vec{x})[\varphi(\vec{x})]$ is $\Sigma_{n+1}^{0}$.

(vi) If $\varphi(\vec{x})$ is $\Sigma_{n}^{0}$ then $(\forall \vec{x})[\varphi(\vec{x})]$ is $\Pi_{n+1}^{0}$.

(vii) A $\Sigma_{1}^{1}$ formula is one of the form $(\exists \vec{X})[\varphi(\vec{X})]$ where $\varphi(\vec{X})$ is an arithmetic formula.

(viii) A $\Pi_{1}^{1}$ formula is one of the form $(\forall \vec{X})[\varphi(\vec{X})]$ where $\varphi(\vec{X})$ is an arithmetic formula.

(ix) A $\Pi_{2}^{1}$ formula is one of the form $(\forall \vec{X})[\varphi(\vec{X})]$ where $\varphi(\vec{X})$ is $\Sigma_{1}^{1}$.

Let $\mathcal{M}=(\mathbb{X}, \mathcal{F},+, \times, 0,1,<)$ be a model for our language. A formula with parameters in $\mathcal{M}$ is one obtainable from a formula in the above language by substituting (constants representing) elements of $\mathbb{X}$ for number variables and elements of $\mathcal{F}$ for set variables. A set $A \subseteq X$ is said to be $\boldsymbol{\Sigma}_{\mathbf{n}}^{\mathbf{0}}$ over $\mathcal{M}$ if it is definable in $\mathcal{M}$ by a formula with parameters in $\mathcal{M}$. (Note the use of boldface because set parameters are allowed.) The notion of $\boldsymbol{\Pi}_{\mathbf{n}}^{\mathbf{0}}$ over $\mathcal{M}$ is defined analogously. A set is called $\boldsymbol{\Delta}_{\mathbf{n}}^{\mathbf{0}}$ over $\mathcal{M}$ if it is both $\boldsymbol{\Sigma}_{\mathbf{n}}^{\mathbf{0}}$ over $\mathcal{M}$ and $\boldsymbol{\Pi}_{\mathbf{n}}^{\mathbf{0}}$ over $\mathcal{M}$.

Definition 6.2. $\quad(i)$ The comprehension scheme is the collection of all universal closures of formulas:

$$
\exists X \forall n[n \in X \leftrightarrow \varphi(n)]
$$

where $\varphi$ is any formula in which $X$ does not occur.

(ii) If $\Gamma$ is a set of formulas, $\Gamma$-comprehension is the comprehension scheme restricted to formulas $\varphi$ in $\Gamma$. 
(iii) The $\Delta_{n}^{0}$ comprehension scheme is the set of all universal closures of formulas of the form

$$
\forall x[\varphi(x) \leftrightarrow \psi(x)] \rightarrow \exists X \forall n[n \in X \leftrightarrow \varphi(n)]
$$

where $\varphi, \psi$ are respectively $\Sigma_{n}^{0}$ and $\Pi_{n}^{0}$ formulas which do not contain the variable $X$. (This scheme is true in a model $\mathcal{M}$ just if any set $\boldsymbol{\Delta}_{\mathbf{n}}^{\mathbf{0}}$ over $\mathcal{M}$ is a real of $\mathcal{M}$.)

(iv) The induction scheme is the collection universal closures of formulas:

$$
[\varphi(0) \wedge \forall n(\varphi(n) \rightarrow \varphi(n+1))] \rightarrow \forall n \varphi(n) .
$$

(v) $I \Gamma$ is the induction scheme restricted to formulas in $\Gamma$.

(vi) The bounding scheme is the collection of formulas:

$$
(\forall a)[(\forall x \leq a)(\exists y) \varphi(x, y) \rightarrow(\exists b)[(\forall x \leq a)(\exists y \leq b) \varphi(x, y)]] .
$$

(vii) $B \Gamma$ is the bounding scheme restricted to formulas in $\Gamma$.

Definition 6.3 (Some subsystems of second order arithmetic).

(i) PA denotes the standard axioms of Peano arithmetic (here the induction scheme is restricted to first order formulas).

(ii) $P^{-}$denotes the usual algebraic axioms of Peano arithmetic (without the induction scheme).

(iii) $R C A_{0}$ (Recursive Comprehension) denotes the axioms of $P^{-}$, $I \Sigma_{1}^{0}$ and $\Delta_{1}^{0}$ comprehension.

(iv) $A C A_{0}$ (Arithmetic Comprehension) is $R C A_{0}$ and arithmetic comprehension.

$\mathcal{M}=(\mathbb{X}, \mathcal{F},+, \times, 0,1,<)$ be a model of $R C A_{0}$. A set $D \in \mathcal{F}$ is called $\mathcal{M}$-finite if it is bounded by some element of $\mathbb{X}$ and otherwise $\mathcal{M}$-infinite. $\mathcal{M}$-finite sets may be coded by elements of $\mathbb{X}$. Let $\langle.,$. be a fixed bijection from $\mathbb{X} \times \mathbb{X}$ onto $\mathbb{X}$ whose graph is $\boldsymbol{\Delta}_{\mathbf{0}}^{\mathbf{0}}$ over $\mathcal{M}$. If $Y \in \mathcal{F}$ and $i \in \mathbb{X}$, let $(Y)_{i}=\{j:\langle i, j\rangle \in Y\}$. In this situation, we say that $Y$ codes the sequence of reals $(Y)_{i}: i \in \mathbb{X}$, and clearly $(Y)_{i} \in \mathcal{F}$ for each $i \in \mathbb{X}$.

Definition 6.4. If $T_{1}$ and $T_{2}$ are theories and $\Gamma$ is a set of sentences then $T_{2}$ is $\Gamma$-conservative over $T_{1}$ if $\forall \varphi\left[\left(\varphi \in \Gamma \wedge T_{2} \vdash \varphi\right) \Rightarrow T_{1} \vdash \varphi\right]$.

Over $R C A_{0}, I \Sigma_{n}$ and $I \Pi_{n}$ are equivalent. $I \Sigma_{n}$ is also equivalent over $R C A_{0}$ to the scheme asserting that every nonempty $\Pi_{n}^{0}$-definable set ( $\Sigma_{n}^{0}$-definable set) has a least element. $B \Sigma_{n+1}$ is stronger than $I \Sigma_{n}$ but not as strong as $I \Sigma_{n+1}$. (See Kaye [1991] or Hájek and Pudlák [1993] for details.)

All models we consider will be countable, i.e., both the base set $\mathbb{X}$ and the second-order part $\mathcal{F}$ will be countable. A model is an $\omega$-model 
if the base set is $\mathbb{N}$ and the operations and relations are the usual ones. Thus, an $\omega$-model $\mathcal{M}$ is completely determined by the family $\mathcal{F}$ of reals of $\mathcal{M}$ and is often identified with $\mathcal{F}$. Full induction clearly holds in all $\omega$-models. $R C A_{0}$ is suitable for formalizing effective proofs (for more details see Simpson [1999]). See the beginning of Section 2 for a description of the $\omega$-models of $R C A_{0}$ and the stronger system $A C A_{0}$. Note that, in particular, the computable sets are the smallest $\omega$-model of $R C A_{0}$, and the arithmetical sets are the smallest $\omega$-model of $A C A_{0}$.

$A C A_{0}$ is arithmetically conservative over $P A$, i.e., any arithmetic sentence is provable from $A C A_{0}$ if and only if it is provable from $P A$.

We will also briefly mention another subsystem of second order arithmetic, $A T R_{0}$ - Arithmetic Transfinite Recursion. The only facts that we will need about $A T R_{0}$ are that the family of arithmetical subsets of $\mathbb{N}$ is not an $\omega$-model of $A T R_{0}$ and that if $\mathcal{M}$ is a model of $A T R_{0}$ and $n$ is a number in $\mathcal{M}$ then $\mathcal{M}$ is closed under the $(n)$-jump. This, $A T R_{0}$ 's definition, and other facts can be found in Simpson [1999].

In this paper, we will work with $\Pi_{1}^{1}$-conservation and arithmetic conservation. We will need the following lemmas and definitions.

Definition 6.5. $\mathcal{M}=(\mathbb{X}, \mathcal{F},+, \times, 0,1,<)$ is an $\omega$-submodel of $\mathcal{M}^{\prime}=\left(\mathbb{X}^{\prime}, \mathcal{F}^{\prime},+^{\prime}, \times^{\prime}, 0^{\prime}, 1^{\prime},<^{\prime}\right)$ if $\mathbb{X}=\mathbb{X}^{\prime},+=+^{\prime}, \times=\times^{\prime}, 0=0^{\prime}$, $1=1^{\prime},<=<^{\prime}$, and $\mathcal{F} \subseteq \mathcal{F}^{\prime}$. In other words, $\mathcal{M}^{\prime}$ may be obtained from $\mathcal{M}$ by just adding reals.

Lemma 6.6. If every countable model of $T_{1}$ is an $\omega$-submodel of some countable model of $T_{2}$ then $T_{2}$ is $\Pi_{1}^{1}$-conservative over $T_{1}$ (both $T_{1}$ and $\mathrm{T}_{2}$ are theories of second order arithmetic).

Proof. Let $\varphi$ be a $\Pi_{1}^{1}$ sentence. If $T_{1}$ does not prove $\varphi$ then there is a countable model $\mathcal{M}$ of $T_{1}+\neg \varphi$. Since we only add reals to get the expansion $\mathcal{M}^{\prime}$, a model of $T_{2}$, and $\neg \varphi$ is $\Sigma_{1}^{1}$, we have that $\mathcal{M}^{\prime}$ is a model of $\neg \varphi$. So $T_{2}$ does not prove $\varphi$

We do not know whether the converse of Lemma 6.6 holds.

The following lemma will be useful throughout the paper.

Lemma 6.7 (Friedman [1976]). Any model $\mathcal{M}$ of $P^{-}$and $I \Sigma_{n}$ is an $\omega$-submodel of some model $\mathcal{M}^{*}$ of $R C A_{0}+I \Sigma_{n}$. Furthermore, $\mathcal{M}^{*}$ may be chosen so that each of its reals is $\boldsymbol{\Delta}_{\mathbf{1}}^{\mathbf{0}}$ over $\mathcal{M}$, and $\mathcal{M}^{*}$ is countable if $\mathcal{M}$ is.

The idea behind the proof is to close under $\Delta_{1}^{0}$-comprehension, which roughly corresponds to closing under Turing reducibility and joins. A proof for $n=1$ can be found in Simpson [1999]; the other cases are similar. In models of second order arithmetic we interpret $A \leq_{T} B$ for 
reals $A, B$ of the model to mean that $A$ is $\Delta_{1}^{0, B}$, i.e., $A$ is definable from the parameter $B$ using a $\Sigma_{1}^{0}$ formula and also using a $\Pi_{1}^{0}$ formula. We will use this without mention throughout the paper.

Definition 6.8. Let $\mathcal{M}=(\mathbb{X}, \mathcal{F},+, \times, 0,1,<)$ be a model of secondorder arithmetic, and let $G \subseteq \mathbb{X}$. Then $\mathcal{M}[G]=(\mathbb{X}, \mathcal{F} \cup\{G\},+, \times, 0,1,<)$, so $\mathcal{M}[G]$ is obtained by adjoining $G$ to the reals of $\mathcal{M}$.

Note that if $\mathcal{M}$ is a model of $P A^{-}$then $M[G]$ is also. (However, $M[G]$ is a model of $R C A_{0}$ only if $G$ is a real of $\mathcal{M}$ (in which case $\mathcal{M}=\mathcal{M}[G])$.) Suppose now that $\mathcal{M}$ is a countable model of $P^{-}$and that $\mathcal{M}[G]$ is a model of $I \Sigma_{n}$. Then, by Lemma 6.7, $\mathcal{M}[G]$ is an $\omega$ submodel of some countable model $\mathcal{M}^{*}$ of $R C A_{0}+I \Sigma_{n}$. We will use this fact repeatedly.

Definition 6.9. We say that $\mathcal{M}$ is topped if $\mathcal{M}$ is countable and satisfies the sentence of second order arithmetic asserting that there is a real of greatest Turing degree. If $D$ is a real of $\mathcal{M}$ such that $\mathcal{M}$ satisfies the formula asserting that every real is Turing reducible to $D$, we say that $\mathcal{M}$ is topped by $D$.

We remark that an $\omega$-model $\mathcal{F}$ is topped if and only if $\mathcal{F}$ contains a real of greatest Turing degree. In general, every model with a real of greatest Turing degree is topped (by that real), but it is not clear that the converse holds because some of the reductions witnessing that a real has greatest Turing degree may be nonstandard.

Lemma 6.10. Assume every topped model of $R C A_{0}+I \Sigma_{n}$ is an $\omega-$ submodel of some countable model of $T$. Then $T$ is arithmetically conservative over $R C A_{0}+I \Sigma_{n}$.

Proof. If $R C A_{0}+I \Sigma_{n}$ does not prove $\varphi$ then there is a countable model $\mathcal{M}$ of $R C A_{0}+I \Sigma_{n}+\neg \varphi$. Remove all of $\mathcal{M}$ 's reals and then apply Lemma 6.7. By Lemma 6.7 the resulting model $\mathcal{M}^{*}$ of $R C A_{0}+I \Sigma_{n}$ is topped since all of its reals are Turing reducible to $\emptyset$. By adding reals to $\mathcal{M}^{*}$ we can get a model $\mathcal{M}^{\prime}$ of $T$. Since we only added and removed reals to get $\mathcal{M}^{\prime}$ and $\varphi$ is arithmetic, it follows that $\mathcal{M}^{\prime}$ is a model of $\neg \varphi$. Hence $\varphi$ is not provable in $T$.

\section{Some STATEMENTS OF SECOND ORDER ARITHMETIC}

Our proof that every computable $k$-coloring of pairs has an infinite low $_{2}$ homogeneous set (Theorem 3.1) proceeded by means of several intermediate results such as the Low Basis Theorem, the existence of a low 2 r-cohesive set, and, basically, the existence of infinite low 2 homogeneous sets for $\Delta_{2}^{0}$ partitions of 1 -tuples (Theorem 3.6. Below we 
consider the corresponding formal statements in second-order arithmetic. These will be useful in proving our conservation results for Ramsey's Theorem. The first statement is Weak König's Lemma and the next three are various forms of Ramsey's Theorem in the language of second order arithmetic.

Recall that in second order arithmetic we say a set $X$ is infinite if $\forall x \exists y[x<y \& y \in X]$, and we can identify binary strings with their Gödel numbers.

Statement 7.1 (Weak König's Lemma). Every infinite tree of binary strings has an infinite branch.

Statement $7.2\left(R T_{k}^{n}\right)$. For every $k$-coloring of $[\mathbb{N}]^{n}$ there is an infinite homogeneous set $H$.

Note that $R T_{k}^{n}$ is equivalent over $R C A_{0}$ to the ostensibly stronger statement that for every infinite set $X$ and every $k$-coloring of $[X]^{n}$ there is an infinite homogeneous set $H$. Similar comments apply to all the versions of Ramsey's Theorem we discuss, since it is provable in $R C A_{0}$ that for every infinite set $X$ there is a bijection from $\mathbb{N}$ onto $X$.

Statement $7.3\left(R T_{<\infty}^{n}\right)$. For every $k, R T_{k}^{n}$.

Statement $7.4(R T)$. For every $n, R T_{<\infty}^{n}$.

Statement $7.5\left(S R T_{k}^{2}\right)$. For every stable $k$-coloring of $[\mathbb{N}]^{2}$ there is an infinite homogeneous set $H$.

Statement $7.6\left(S R T_{<\infty}^{2}\right)$. For every $k, S R T_{k}^{2}$.

The following statement defines cohesiveness with respect to a sequence of sets and asserts the existence of a cohesive set in this framework.

Statement $7.7(C O H)$. For any sequence of sets $\left(R_{i}\right)_{i \in \mathbb{N}}$ there is an infinite set $A$ such that for all $i$, either $A \subseteq{ }^{*} R_{i}$ or $A \subseteq \subseteq^{*} \bar{R}_{i}$. (Such a set $A$ is called $\vec{R}$-cohesive.) $X \subseteq^{*} Y$ means there is a $k$ such that for all $x$, if $x \in X$ then either $x \in Y$ or $x \leq k$. (To say that $\left(R_{i}\right)_{i \in \mathbb{N}}$ is a sequence of sets means that there is a set $R$ with $R_{i}=\{j:\langle i, j\rangle \in R\}$ for each $i$.)

$\mathrm{COH}$ can be considered as a very strong form of $R T_{2}^{1}$. It says for every infinite sequence of 2 -colorings of $[\mathbb{N}]^{1}$ there is an infinite set which is homogeneous modulo a finite set for each coloring. If the terms of the sequence $\vec{R}$ are exactly the primitive recursive subsets of $\mathbb{N}$ then the $\vec{R}$-cohesive sets are precisely the p-cohesive sets. (See Definition 3.2 or Jockusch and Stephan [1993].) Note that any $\omega$-model 
of $R C A_{0}$ contains a sequence of sets consisting of exactly the primitive recursive sets. It follows that any $\omega$-model of $R C A_{0}+C O H$ contains a $p$-cohesive set. Similarly, if the terms of the sequence $\vec{R}$ are exactly the computable subsets of $\mathbb{N}$ then the $\vec{R}$-cohesive sets are precisely the r-cohesive sets. (See Definition 3.2 or Jockusch and Stephan [1993].) It is known that any $\omega$-model of $W K L_{0}$ contains a sequence of sets containing all computable subsets of $\mathbb{N}$ (and possibly more), so that any $\omega$-model of $W K L_{0}+C O H$ contains an $\mathrm{r}$-cohesive set (see Sections 8.4 and 8.5).

If the colorings of 1 -tuples $R_{i}$ are replaced by colorings of $n$-tuples, the resultings notions of cohesiveness are studied in Hummel and Jockusch [n.d.] The next two statement are phrasings of Theorems 3.6 and 3.7 in the language of second order arithmetic. (The superscript stands for $\Delta_{2}^{0}$ and the subscript stands for the number of such sets.) We will shortly see that over $R C A_{0}$ they are equivalent to the appropriate statement about stable colorings but in some cases this form will prove to be slightly easier to work with.

Statement $7.8\left(D_{2}^{2}\right)$. For every function $f(x, s)$, if for all $x$ and $s$, $f(x, s)<2$, and for all $x \lim _{s} f(x, s)$ exists, then there is an infinite set $G$ and $j<2$ such that for all $x \in G, \lim _{s} f(x, s)=j$.

Statement $7.9\left(D_{<\infty}^{2}\right)$. For every $k$ and for every function $f(x, s)$, if for all $x$ and $s, f(x, s)<k$, and for all $x, \lim _{s} f(x, s)$ exists then there is an infinite set $G$ and $j<k$ such that for all $x \in G, \lim _{s} f(x, s)=j$.

We can consider $F(x)=\lim _{s} f(x, s)$ as giving a $k$-coloring of $\mathbb{N}$ and $G$ as a homogeneous set for this coloring. Hence we can consider these statements as very strong forms of $R T_{2}^{1}$ and $R T_{<\infty}^{1}$.

\subsection{Some lemmas about $S R T_{2}^{2}, D_{2}^{2}, S R T_{<\infty}^{2}$, and $D_{<\infty}^{2}$.}

Lemma 7.10. $R C A_{0} \vdash S R T_{2}^{2} \Leftrightarrow D_{2}^{2}$

Proof. Assume $S R T_{2}^{2}$ and let $f(i, j)$ be given. Color $\{i, j\}$ red for $i<j$ iff $f(i, j)=0$. A homogeneous set for this coloring is the desired set to satisfy $D_{2}^{2}$.

Assume $D_{2}^{2}$. Let $\mathcal{C}$ be a given stable 2-coloring and define $f(i, j)=\mathcal{C}(\{i, j\})$ if $i<j$ and arbitrarily otherwise. The set $G$ given via $D_{2}^{2}$ from $f$ can be easily used to find an infinite set $H \subseteq G$ which is homogeneous for $\mathcal{C}$.

Lemma 7.11. $R C A_{0} \vdash R T_{2}^{2} \Leftrightarrow\left(C O H \& S R T_{2}^{2}\right)$.

Proof. Let $\mathcal{M}=(\mathbb{X}, \mathcal{F}, \ldots)$ be a model of $C O H$ and $S R T_{2}^{2}$. Fix a 2 -coloring $\mathcal{C} \in \mathcal{F}$ of $[\mathbb{X}]^{2}$ into the colors red and blue. For 
$i \in \mathbb{X}$ let $R_{i}=\{j>i:\{i, j\}$ has color red $\}$, and note that $\vec{R}=\left\{\langle i, j\rangle j \in R_{i}\right\} \in \mathcal{F}$. Use $C O H$ to get an $\vec{R}$-cohesive set $A$. Since $A$ is infinite, there is 1-1 onto map $g: \mathbb{X} \rightarrow A$ coded as a real of $\mathcal{M}$. Create a coloring $\mathcal{C}^{\prime}$ as follows: $\mathcal{C}^{\prime}$ colors $\{i, j\}$ red iff $\mathcal{C}$ colors $\{g(i), g(j)\}$ red. Since $A$ is $\vec{R}$-cohesive, $\mathcal{C}^{\prime}$ is stable. If $H$ is homogeneous for $\mathcal{C}^{\prime}$ then $g(H)$ is homogeneous for $\mathcal{C}$.

Assume $R T_{2}^{2}$. Then clearly $S R T_{2}^{2}$ holds. Given a sequence $R_{i}$, if $i<j$, then color $\{i, j\}$ red iff $j \in R_{i}$. Every homogeneous set for this 2-coloring is $\vec{R}$-cohesive.

Lemma 7.12. $R C A_{0} \vdash S R T_{<\infty}^{2} \Leftrightarrow D_{<\infty}^{2}$.

Proof. Similar to the proof of Lemma 7.10 .

Lemma 7.13. $R C A_{0} \vdash R T_{<\infty}^{2} \Leftrightarrow C O H+S R T_{<\infty}^{2}$.

Proof. Similar to the proof of Lemma 7.11.

\subsection{Induction: 2 vs. any finite number of colors.}

Lemma 7.14. The following are theorems of $R C A_{0}$.

(i) For all $k \geq 2$ and all $n, R T_{k}^{n}$ implies $R T_{k+1}^{n}$.

(ii) For all $k \geq 2, S R T_{k}^{2}$ implies $S R T_{k+1}^{2}$.

Proof. We will just prove $i$. We reason in $R C A_{0}$. Let $\mathcal{C}:[\mathbb{N}]^{n} \rightarrow\{0,1, \ldots, k\}$ be a $(k+1)$-coloring of $[\mathbb{N}]^{n}$. $\mathcal{C}$ induces a $k$-coloring of $[\mathbb{N}]^{n}$; for $Y \in[\mathbb{N}]^{n}$, $\mathcal{C}^{\prime}(Y)=\mathcal{C}(Y)$ unless $\mathcal{C}(Y)=k$, in which case $\mathcal{C}^{\prime}(Y)=k-1$. Using $R T_{k}^{n}$, let $H$ be an infinite homogeneous set for $\mathcal{C}^{\prime}$. If $\mathcal{C}^{\prime}\left([H]^{n}\right) \neq k-1$, $H$ is homogeneous for $\mathcal{C}$. Otherwise use $\mathcal{C}$ to induce a coloring $\mathcal{C}^{\prime \prime}$ on $H$; for $Y \in[H]^{n}$, let $\mathcal{C}^{\prime \prime}(Y)=0$ if $\mathcal{C}(Y)=k-1$ and $\mathcal{C}^{\prime \prime}(Y)=1$ if $\mathcal{C}(Y)=k$. Every homogeneous set for $\mathcal{C}^{\prime \prime}$ is homogeneous for $\mathcal{C}$.

Corollary 7.15. ( $i)$ For any $k \geq 2, R T_{k}^{2}$ is equivalent to $R T_{2}^{2}$ over $R C A_{0}$.

(ii) For any $k \geq 2, S R T_{k}^{2}$ is equivalent to $S R T_{2}^{2}$ over $R C A_{0}$.

We will later see that $R T_{<\infty}^{2}$ is strictly stronger than $R T_{2}^{2}$ over $R C A_{0}$ (Corollary 11.5) and that $S R T_{<\infty}^{2}$ is strictly stronger than $S R T_{2}^{2}$ over $R C A_{0}$ (Theorem 11.4). By work of Simpson [1999] (see Corollary 2.6), it is known that for $n \geq 3$ and $k \geq 2, R T_{<\infty}^{n}$ and $R T_{k}^{n}$ are each equivalent to to $A C A_{0}$ over $R C A_{0}$. Thus, the logical strength of $R T_{k}^{n}$ is independent of $n$ and $k$ for $n \geq 3,2 \leq k \leq \infty$.

\section{WEAK KÖNIG'S LEMMA}

8.1. Low Basis Theorem and conservation. Weak König's Lemma is the fact that every infinite tree of binary strings has an infinite 
branch. As we noted in the Introduction, the Low Basis Theorem will play an important role in our work. Here we will consider it as a theorem about the effective content of Weak König's Lemma.

Theorem 8.1 (Jockusch and Soare [1972]). Every infinite computable tree of binary strings has an infinite low path $P$ (i.e., $P^{\prime} \leq_{T} 0^{\prime}$ ).

Leo Harrington, in unpublished work, used the idea of the proof of the Low Basis Theorem to produce a notion of forcing over models of second order arithmetic to prove the following technical lemma (Lemma 8.2 which then in turn yields the following conservation result (Corollary 8.4). See Simpson [1999] for the details.

Lemma 8.2 (Harrington). If $\mathcal{M}$ is a model of $R C A_{0}, T \in \mathcal{F}$ and $T$ codes an $\mathcal{M}$-infinite tree of binary strings then there is a $P \subset \mathbb{X}$ such that $\mathcal{M}[P]$ is a model of $I \Sigma_{1}$ and $P$ is an $M[P]$-infinite path through $T$.

Theorem 8.3 (Harrington). Every countable model of $R C A_{0}$ is an $\omega-$ submodel of some countable model of $W K L_{0}$.

Proof of Theorem 8.3 from Lemma 8.2. Let $\mathcal{M}=(\mathbb{X}, \mathcal{F}+, \times, 0,1,<)$ be a model of $R C A_{0}$. Choose some $T \in \mathcal{F}$ coding an $\mathcal{M}$-infinite tree of binary strings. Apply Lemma 8.2 to get $\mathcal{M}^{\prime}$. Then apply Lemma 5.3 to get a model $\mathcal{M}^{\prime \prime}$ of $R C A_{0}$. Iterate the process infinitely many times to produce a sequence of models $\mathcal{M}_{n}=\left(\mathbb{X}, \mathcal{F}_{n},+, \times, 0,1,<\right)$ with $\mathcal{F}_{0} \subseteq F_{1} \subseteq \mathcal{F}_{2} \ldots$, and let $\mathcal{M}_{\omega}=\left(\mathbb{X}, \cup_{n} \mathcal{F}_{n},+, \times, 0,1,<\right)$ ensuring that for every such $T \in \cup_{n} \mathcal{F}_{n}$ an $\mathcal{M}_{\omega}$-infinite path is added to $\cup_{n} \mathcal{F}_{n}$. Then $\mathcal{M}_{\omega}$ is a countable model of $W K L_{0}$ which has $\mathcal{M}$ as an $\omega$-submodel.

Corollary 8.4 (Harrington). $W K L_{0}$ is $\Pi_{1}^{1}$-conservative over $R C A_{0}$.

Proof. This is immediate from Theorem 8.3 and Lemma 6.6.

We will need some results analogous to Lemma 8.2, Theorem 8.3 and Corollary 8.4 for stronger forms of induction.

Lemma 8.5. If $\mathcal{M}$ is a model of $R C A_{0}+I \Sigma_{2}$, $T$ is a real of $\mathcal{M}$ which codes an $\mathcal{M}$-infinite tree of binary strings, then there is a set $P$ of numbers of $\mathcal{M}$ such that $\mathcal{M}[P]$ is a model of $I \Sigma_{2}$ and $P$ is an $\mathcal{M}[P]-$ infinite path through $T$.

Corollary 8.6. Every countable model of $R C A_{0}+I \Sigma_{2}$ is an $\omega$ submodel of some countable model of $W K L_{0}+I \Sigma_{2}$.

Proof of Corollary 8.6 from Lemma 8.5. This is entirely analogous to the proof of Theorem 8.3 from Lemma 8.2 . 
Lemma 8.7. Assume $\mathcal{M}$ is a topped model of $R C A_{0}+I \Sigma_{3}$. Suppose that $T$ is a real of $\mathcal{M}$ and $T$ codes an $\mathcal{M}$-infinite tree of binary strings. Then there is a set $P$ of numbers of $\mathcal{M}$ such that $P$ is an $\mathcal{M}[P]$-infinite path through $T$ and $\mathcal{M}[P]$ is a model of $I \Sigma_{3}$.

Note that after applying the proof of Lemma 8.7 to $\mathcal{M}^{\prime}$ above, the resulting model is still topped (by $P \oplus D$ ). So we can repeatedly apply Lemma 8.7 as above to get the following corollary.

Corollary 8.8. Every topped model of $R C A_{0}+I \Sigma_{3}$ is an $\omega$-submodel of some countable model of $W K L_{0}+I \Sigma_{3}$.

The proofs of Lemmas 8.5 and 8.7 follow in Sections 8.2 and 8.3 .

8.2. $I \Sigma_{2}$ Conservation and Weak König's Lemma. Fix a model $\mathcal{M}=(\mathbb{X}, \mathcal{F},+, \times, 0,1,<)$ of $R C A_{0}+I \Sigma_{2}$. We will call $\mathcal{M}$ the "ground model." Let $T$ be an $\mathcal{M}$-infinite tree of binary strings in $\mathcal{F}$. We will add a set $P$ such that $P$ is an $\mathcal{M}[P]$-infinite path in $T$ and $\mathcal{M}[P]$ satisfies $I \Sigma_{2}$.

Except for the set $P$, we will assume in this subsection that all numbers, strings, and sets mentioned are in the ground model.

We force over $\mathcal{M}$ using conditions $C \in \mathcal{F}$ where $C$ codes an $\mathcal{M}$ infinite subtree of $T$. $C$ extends $C^{*}$ if $C$ is an $\mathcal{M}$-infinite subtree of $C^{*}$. We will need the following definitions and lemmas in our proof.

If $\tau \in C$ then let $C_{\tau}=\{\sigma \in C: \sigma \preceq \tau$ or $\tau \preceq \sigma\}$. If $C$ is an $\mathcal{M}_{-}$ infinite tree then for all $l$ there is a $\tau \in C$ of length $l$ such that $C_{\tau}$ is an $\mathcal{M}$-infinite subtree of $C$. We define forcing for certain formulas of low quantifier complexity. For the rest of this proof, the word "formula" will mean a formula of second-order arithmetic with parameters from $\mathcal{M}$ and no free variables other than the ones displayed.

Definition 8.9. $\quad(i)$ We say $C$ forces $(\exists \vec{x})(\psi(\vec{x}, G))$ if there exists $l$ such that for all $\tau \in C$ of length $l$ there is a $\vec{w} \in X^{n}$ such that $\tau$ forces $\psi(\vec{w}, \tau)$. (Forcing of $\Delta_{0}^{0}$ formulas by strings was defined in Definition 5.1. Here we use the same definition but allow parameters from $\mathcal{M}$ as constants.)

(ii) We say $C$ forces $(\forall \vec{y})(\exists \vec{x})(\psi(\vec{x}, \vec{y}, G))$ if for all $\vec{w} \in M^{n}, C$ forces $(\exists \vec{x})(\psi(\vec{x}, \vec{w}, G))$.

Note that forcing implies truth for all the formulas considered above. Thus, for any formula $\theta(G)$ which is $\Sigma_{1}^{0}$ or $\Pi_{2}^{0}$ and any condition $C$ and any $\mathcal{M}[P]$-infinite path $P$ through $C$, if $C$ forces $\theta(G)$, then $\mathcal{M}[P] \models \theta(P)$. The corresponding statement also holds for $\Delta_{0}^{0}$ formulas and strings. Note that, at these levels, "forcing implies truth" holds 
for all $P \subseteq \mathbb{X}$, and there is no requirement that $P$ must be generic in any sense.

Lemma 8.10. Let $\mathcal{M}$ be a model of $R C A_{0}$.

( $i$ ) Let $\psi(\vec{x}, G)$ be a $\Delta_{0}^{0}$ formula. Then the relation " $\tau$ is a binary string and $\vec{w}$ is a sequence of parameters and $\tau$ forces $\psi(\vec{w}, G)$ " is $\boldsymbol{\Delta}_{\mathbf{0}}^{\mathbf{0}}$ over $\mathcal{M}$.

(ii) Let $\psi(\vec{y}, G)$ be a $\Sigma_{1}^{0}$ formula and let $C$ be a condition. Then $\left\{\vec{w} \in M^{n}: C\right.$ forces $\left.\psi(\vec{w}, G)\right\}$ is $\Sigma_{1}^{0}$ over $\mathcal{M}$.

(iii) Let $\psi(\vec{y}, G)$ be a $\Pi_{2}^{0}$ formula and let $C$ be a condition. Then $\left\{\vec{w} \in M^{n}: C\right.$ forces $\left.\psi(\vec{w}, G)\right\}$ is $\Pi_{2}^{\mathbf{0}}$ over $\mathcal{M}$.

Proof. Part (i) is proved by induction on the complexity of the $\Delta_{0}^{0}$ formula $\psi(\vec{x}, G)$.

Then part (ii) follows from part(i), and part (iii) follows from part (ii).

Let $\psi(\vec{x}, G)$ be a $\Delta_{0}^{0}$ formula. We say that the condition $C$ forces $\neg(\exists \vec{x})(\psi(\vec{x}, G))$ if for all strings $\tau \in C$ and $\vec{w} \in \mathbb{X}^{n} \tau$ does not force $\psi(\vec{w}, G)$. Since conditions are $\mathcal{M}$-infinite and for any $\Delta_{0}^{0}$ formula $\delta$ any sufficiently long binary string $\tau$ coded in $\mathcal{M}$ forces either $\delta$ or $\neg \delta$, it is easily seen that forcing implies truth here. Finally, we say that $C$ forces $\neg(\forall \vec{y})(\exists \vec{x}) \theta\left(\psi(\vec{x}, \vec{y}, G)\right.$ for $\psi \Delta_{0}^{0}$ if there is a tuple $\vec{u} \in \mathcal{M}$ such that $C$ forces $\neg \exists \vec{x} \psi(\vec{x}, \vec{u}, G)$. Again, it is clear that forcing implies truth.

Lemma 8.11. If $\theta(G)$ is a $\Pi_{2}^{0}$ formula and $C$ is a condition which does not force $\theta(G)$, then there is an extension $C^{*}$ of $C$ such that $C^{*}$ forces $\neg \theta(G)$.

Proof. Let $\theta(G)$ be $(\forall \vec{y})(\exists \vec{x}) \psi(\vec{x}, \vec{y}, G)$ where $\psi$ is $\Delta_{0}^{0}$. Since $C$ does not force $(\forall \vec{y})(\exists \vec{x}) \psi(\vec{x}, \vec{y}, G)$ there is a $\vec{w} \in M^{n}$ such that $C$ does not force $(\exists \vec{x})(\psi(\vec{x}, \vec{w}, G))$. Fix such a $\vec{w}$.

Since $C$ does not force $(\exists \vec{x})(\psi(\vec{x}, \vec{w}, G))$, it follows that for all $l \in \mathbb{X}$ there exists a $\tau \in C$ of length $l$ such that $\tau$ does not force $\psi(\vec{v}, \vec{w}, \tau)$.

Let $C^{*}$ be the subset of $C$ formed by taking all such $\tau$. It is easily seen that $C^{*}$ is a condition. Then $C^{*}$ forces $\neg(\exists \vec{x})(\psi(\vec{x}, \vec{w}, G))$.

8.2.1. Preserving $I \Sigma_{2}$. For all $\theta(x, G)$, a $\Pi_{2}^{0}$-formula, we want to ensure either $\mathcal{M}[G] \models \theta(a, P)$ for every number $a$ of $\mathcal{M}$ or else there is a least $b$ such that $\mathcal{M}[G] \models \neg \theta(b, P)$. Hence we are ensuring that every nonempty set which is $\boldsymbol{\Sigma}_{\mathbf{2}}^{\mathbf{0}}$ over $\mathcal{M}[G]$ has a least element.

Fix a condition $C$. Consider the set $S$ of $c$ such that $C$ does not force $\theta(c, G)$. By Lemma $8.10, S$ is $\Sigma_{\mathbf{2}}^{0}$ over $\mathcal{M}$. If $S=\emptyset, C$ forces $\theta(a, G)$ for every $a \in \mathcal{M}$. If $S \neq \emptyset$, it has a least element $b$ by $I \Sigma_{2}$ in 
the ground model $\mathcal{M}$. Then $C$ forces $\theta(c, G))$ for each $c<b$ and so does each extension of $C$. By Lemma 8.11, there is an extension $C^{*}$ of $C$ which forces $\neg \theta(b, G)$. Hence $b$ is the least element of $\mathbb{X}$ satisfying $\theta(x, G)$ for any path $P$ through $C^{*}$.

8.2.2. Putting it all together. Above we showed how to ensure that a single nonempty $\boldsymbol{\Pi}_{\mathbf{2}}^{\mathbf{0}}$ subset of $\mathcal{M}[G]$ has a least element. It is now a routine matter to do this for all such subsets simultaneously.

Let $\theta_{i}(x, G)_{i \in \mathbb{N}}$ be a listing of $\Pi_{2}^{0}$-formulas. Let $f: \mathbb{N} \rightarrow \mathbb{X}$ be a bijection.

We will construct conditions $C_{s}, s \in \mathbb{N}$, such that there is a unique $M[P]$-infinite path $P$ through $\cap_{s}\left\{C_{s}\right\}$, and furthermore, adding $P$ to the reals of $\mathcal{M}$ preserves $I \Sigma_{2}$. Let $C_{0}=T$.

Stage $s+1$ : Let the condition $C_{s}$ be given. Let $\theta_{s}$ be $(\forall \vec{y}) \psi_{s}(x, \vec{y}, G)$, where $\psi_{s}(x, \vec{y}, G)$ is $\Sigma_{1}^{0}$. Using the procedure in Section 8.2.1 find a condition $C^{*}$ extending $C_{s}$ such that either $C^{*}$ forces $\left(\theta_{s}(c, G)\right)$ for all $c \leq a_{s}$ or for some $b, C^{*}$ forces $\left.\theta_{s}(c, G)\right)$ for each $c<b$ there is a tuple $\vec{w}$ such that $C^{*}$ forces $\neg \psi_{s}(c, \vec{w}, G)$. Let $C_{s+1}$ be $C_{\tau_{s}}^{*}$ where $\tau_{s}$ is of length $\geq f(s)$ and $C_{\tau_{s}}^{*}$ is $\mathcal{M}$-infinite.

Let $P=\cup_{s} \tau_{s}$. It is easily seen that $P$ is a branch of each tree $C_{s}$. To show that $I \Sigma_{2}$ holds in $M[P]$ it suffices to show that whenever a sentence which is $\Pi_{2}$ or $\Sigma_{1}^{0}$ is forced by a condition $C$ having $P$ as a path, then it is true of $P$. This is clear from the definition of forcing for such sentences.

8.3. The Proof of Lemma 8.7. This will be similar to the proof in Section 8.2 except that dealing with $I \Sigma_{3}$ introduces some additional technical complications. Fix a topped model $\mathcal{M}=(\mathbb{X}, \mathcal{F},+, \times, 0,1,<)$ of $R C A_{0}+I \Sigma_{3}$, and suppose that $\mathcal{M}$ is topped by $D \in \mathcal{F}$. Let $T$ be an $\mathcal{M}$-infinite tree of binary strings in $\mathcal{F}$. We will add a set $P$ such that $P$ is an $\mathcal{M}[P]$-infinite path in $T$ preserving $I \Sigma_{3}$.

Except for the set $P$, we will assume in this subsection that all numbers and sets mentioned are in the ground model. As before we force over $\mathcal{M}$ using conditions $C \in \mathcal{M}$ where $C$ is an $\mathcal{M}$-infinite subtree of $T$.

8.3.1. Forcing $\Sigma_{3}^{0}$ statements. We say $C$ forces a $\Sigma_{3}^{0}$ statement $(\exists \vec{x}) \delta(\vec{x}, G)$, where $\delta(\vec{x}, G)$ is a $\Pi_{2}^{0}$ statement, if there exists some sequence of parameters $\vec{w}$ such that $C$ forces $\delta(\vec{w}, G)$. We know from Lemma 8.10 that if $\theta(x, G)$ is a $\Pi_{2}^{0}$ statement and $C$ is a condition, then $\{a: C$ forces $\theta(a, G)\}$ is $\boldsymbol{\Pi}_{\mathbf{2}}^{\mathbf{0}}$ over $\mathcal{M}$. It follows easily that forcing is $\boldsymbol{\Sigma}_{\mathbf{3}}^{\mathbf{0}}$ in the analogous sense for $\Sigma_{3}^{0}$ statements. However, there is a problem in handling negations of $\Sigma_{3}^{0}$ statements. If we define forcing for such statements in 
analogy with the definition of forcing for negations of $\Pi_{2}^{0}$ statements just before the statement of Lemma 8.11, then it is not clear that the analogue of Lemma 8.11 will hold. Instead we use the traditional definition of forcing for negation so that the analogue of Lemma 8.11 is trivially true. Specifically, we say that a condition $C$ forces $\neg \theta(G)$, where $\theta(G)$ is a $\Sigma_{3}^{0}$ formula, if no condition $C^{\prime}$ extending $C$ forces $\theta(G)$. The following definition and lemma show that forcing and truth agree for sufficiently generic sets.

Definition 8.12. (i) If $P \subseteq \mathbb{X}$ and $\theta(G)$ is a formula, then $P$ forces $\theta(G)$ if there is a condition $C$ which has $P$ as a branch and forces $\theta(G)$.

(ii) A set $P \subseteq \mathbb{X}$ is 2-generic over $\mathcal{M}$ if for each $\Pi_{2}^{0}$ formula $\psi(G)$, either $P$ forces $\psi(G)$ or $P$ forces $\neg \psi(G)$.

Lemma 8.13. If $\theta(G)$ is $\Sigma_{3}^{0}$ and $P$ is 2-generic over $\mathcal{M}$ and $P$ forces $\neg \theta(G)$, then $\mathcal{M}=\neg \theta(P)$.

Proof. This is a standard argument.

Let $C$ be a condition such that $P$ is a path through $C$ and $C$ forces $\neg \theta(G)$. Let $\theta(G)$ be $\exists \vec{x} \psi(\vec{x}, G)$ where $\psi$ is $\Pi_{2}^{0}$. We must show that $\mathcal{M} \models \neg \psi(\vec{w}, P)$ for any sequence $\vec{w}$ of parameters from $\mathcal{M}$ of the appropriate length. Since $P$ is 2 -generic over $\mathcal{M}, P$ forces either $\psi(\vec{w}, G)$ or $\neg \psi(\vec{w}, G)$. Suppose for the moment that $P$ forces $\psi(\vec{w}, G)$, and let $C^{*}$ be a condition such that $P$ is a branch of $C^{*}$ and $C^{*}$ forces $\psi(\vec{w}, G)$. Then $C \cap C^{*}$ is a condition which extends $C$ and forces $\psi(\vec{w}, G)$, which contradicts the hypothesis that $C$ forces $\neg \theta(G)$. This contradiction shows that $P$ forces $\neg \psi(\vec{w}, G)$. Since forcing implies truth for $\psi, \mathcal{M} \models \neg \psi(\vec{w}, G)$, as needed.

Unfortunately, it is not clear that forcing for negations of $\Sigma_{3}^{0}$ statements is $\Sigma_{k}^{0}$-definable in $\mathcal{M}$ for any $k$ since its definition involves a set quantifier (over conditions $\mathcal{C}^{\prime}$ ). We do not know how to handle this problem in general, but here we handle it by requiring that $\mathcal{M}$ be topped, as in Definition 6.9. Fix a set $D$ such that $\mathcal{M}$ is topped by $D$, i.e., $\mathcal{M}$ satisfies the second-order statement asserting that $D$ has greatest Turing degree among all reals.

Lemma 8.14. Fix a condition $C$ and a $\Sigma_{3}^{0}$ formula $\delta(y, G)$. Let $S=\{a \in \mathbb{X}: C$ forces $\neg \delta(a, G)\}$. Then $S$ is $\boldsymbol{\Pi}_{\mathbf{3}}^{\mathbf{0}}$ over $\mathcal{M}$.

Proof. Note that $a \in S$ iff there does not exist $e$ such that $\{e\}^{D}$ is a condition $C^{*}$ extending $C$ which forces $\delta(a, G)$. Note that it is a $\Sigma_{3}^{0}$ predicate of a condition $C^{*}$ and $a$ to assert that $C^{*}$ forces $\delta(a, G)$. 
Routine quantifier manipulations then show that the condition $a \in S$ is $\Pi_{3}^{0, D}$ and hence $\boldsymbol{\Pi}_{\mathbf{3}}^{\mathbf{0}}$ over $\mathcal{M}$.

Of course, if the statement in Lemma 8.14 fails to hold, then $C$ forces $\neg(\exists \vec{x}) \delta(\vec{x})$.

8.3.2. Preserving $I \Sigma_{3}$. This section is the same as Section 8.2.1 except that the formula $\theta(x, G)$ is now $\Pi_{3}^{0}$ instead of $\Pi_{2}^{0}$, and correspondingly the set $S$ is $\Sigma_{3}^{0}$ instead of $\Sigma_{2}^{0}$. One must also assume that $P$ is 2 -generic over $\mathcal{M}$ and apply Lemma 8.14 to go from forcing to truth.

8.3.3. Putting it all together. This section is the same as Section 8.2 .2 except that one considers $\Pi_{3}^{0}$ formulas instead of $\Pi_{2}^{0}$ formulas, and also one must ensure that the constructed set $P$ is $2-$ generic over $\mathcal{M}$. Actually, this is already achieved by the given construction, or alternatively one may easily add steps to achieve this.

\subsection{Scott Sets and $P A^{X}$ Degrees.}

Definition 8.15. A $S$ cott set $\mathcal{S}$ is a nonempty family of reals which is closed under join such that if $X \in \mathcal{S}$ and $T$ is an infinite tree of binary strings computable in $X$ then $T$ has an infinite branch in $\mathcal{S}$.

The Scott sets are precisely the $\omega$-models of $W K L_{0}$ (see Simpson [1999]).

Let $\left\{\varphi_{n}\right\}_{n \in \mathbb{N}}$ be a computable listing of all sentences in the language of $P A$. Define a computable tree $\operatorname{Tr}$ as follows: $\sigma \in \operatorname{Tr}$ iff for all $n<|\sigma|$, if $P A \vdash \varphi_{n}$ with a proof of Gödel number $\leq|\sigma|$ then $\sigma(n)=1$ and if $P A \vdash \neg \varphi_{n}$ with a proof of Gödel number $\leq|\sigma|$ then $\sigma(n)=0$. Every completion of $P A$ is an infinite path though $\operatorname{Tr}$. Every infinite path $P$ though $\operatorname{Tr}$ computes a completion of $P A$, by effectivizing the proof of Lindenbaum's Lemma. (We build the completion $T$ stagewise in $P$. Given $\theta_{0} \ldots \theta_{s}$ in $T$. Let $\varphi_{m}$ be the formula $\theta_{0} \wedge \theta_{1} \ldots \wedge \theta_{s} \wedge \varphi_{s+1} \Longrightarrow 0=1$. If $m \in P$ then let $\theta_{s+1}=\neg \varphi_{s+1}$; otherwise let $\theta_{s+1}=\varphi_{s+1}$. Notice that this can be done uniformly.) Let $T$ be a completion of $P A$. We call a degree $\mathbf{d}$ a $P A$ degree if there is some completion of $P A$ computable in $\mathbf{d}$ (or, equivalently, if $\mathbf{d}$ computes an infinite path though $\mathrm{Tr}$ ).

A set $S \subseteq \mathbb{N}$ is binumerable in $T$ if there is a formula $\varphi(x)$, with no free variable other than $x$, such that $S$ is the set of $n$ such that $\varphi(n)$ is provable in $T$. The following theorem shows that there is a Scott set, $\mathcal{S}_{T}$, which is uniformly computable from $T$.

Theorem 8.16 (Scott [1962]). Let $T$ be a completion of PA. Then the family $\mathcal{S}_{T}$ of all sets binumerable in $T$ is a Scott set. 
Sketch of the proof. Suppose $A=\{n: T \vdash \varphi(n)\}$. First suppose that $B=\{n: T \vdash \psi(n)\}$. Then

$$
A \oplus B=\{n: T \vdash(\exists m)((\varphi(m) \wedge n=2 m) \vee(\psi(m) \wedge n=2 m+1)) .
$$

Now we show that $\mathcal{S}_{T}$ is closed downwards under Turing reducibility. Assume $A$ is as above and $C=\Phi^{A}$ for some Turing functional $\Phi$. Then

$$
\begin{gathered}
C=\left\{n: T \vdash(\exists \sigma)\left(\sigma \subseteq \chi_{A} \wedge \Phi_{|\sigma|}^{\sigma}(n)=1\right.\right. \\
\left.\left.\wedge\left(\forall \sigma^{\prime}\right)\left(\sigma^{\prime} \nsubseteq \chi_{A} \vee \Phi_{\left|\sigma^{\prime}\right|}^{\sigma^{\prime}}(n) \uparrow \vee\left|\sigma^{\prime}\right|>|\sigma|\right)\right)\right\}
\end{gathered}
$$

This formula says that $\sigma$ is the initial segment of $A$ of least length such that $\Phi_{|\sigma|}^{\sigma}(n)=1$. Hence $C \in \mathcal{S}_{T}$. Assume that $A$ codes a tree in $2^{\omega}$. The characteristic function of the following set is a path through $A$.

$$
\begin{aligned}
& \{n: T \vdash(\exists \sigma)(|\sigma|=n+1 \wedge \varphi(\ulcorner\sigma\urcorner) \wedge \sigma(n)=1 \wedge(\forall y<|\sigma|)(\sigma(y)=0 \\
& \text { iff } \left.\left.\left.(\forall \tau \supseteq(\sigma\lceil y)\urcorner)\left(\exists \tau^{\prime} \supseteq(\sigma\lceil y)\urcorner\right)\left(\varphi(\ulcorner\tau\urcorner) \wedge \varphi\left(\left\ulcorner\tau^{\prime}\right\urcorner\right) \wedge\left|\tau^{\prime}\right| \geq|\tau|\right)\right)\right)\right\}
\end{aligned}
$$

(This formula says that our path branches right at any level if for every finite extension to the left there is a longer one to the right.)

Let $T$ be a complete extension of $P A$ of low degree, which exists by the Low Basis Theorem. $\mathcal{S}_{T}$ is a Scott set in which all the sets are low, in fact, uniformly low.

This can all be relativized to any set $X$. Just add a new unary predicate $P$ to the language of $P A$ and axioms $P(n)$ if $n \in X$ and $\neg P(n)$ if $n \notin X$, and allow $P$ to occur in the induction scheme. Call the resulting theory $P A^{X}$. Hence using the above we get a tree $\operatorname{Tr}^{X}$ such every infinite path though $\operatorname{Tr}^{X}$ computes a completion of $P A^{X}$ and every completion of $P A^{X}$ is an infinite path though $\operatorname{Tr}^{A}$. A degree d is a $P A^{X}$ degree if there is some completion of $P A^{X}$ computable in d. Clearly $X$ is binumerable in a completion of $P A^{X}$. Hence Scott's theorem implies that for each completion $T$ of $P A^{X}$ there is a Scott set, $\mathcal{S}_{T}$, such that $\mathcal{S}_{T}$ is uniformly computable from $T$ and $X \in \mathcal{S}$.

Recall that in Definition 4.1 we defined $\mathbf{a}>>\mathbf{b}$ to mean that every partial $\{0,1\}$-valued $\mathbf{b}$ computable function has a total $\mathbf{a}-$-computable function. The following well-known result shows that this is equivalent to Simpson's original definition.

Lemma 8.17. Let $\mathbf{a}$ and $\mathbf{b}$ be degrees. The following are equivalent:

(i) $\mathbf{a}>>\mathbf{b}$

(ii) $\mathbf{a}$ is a $P A^{\mathbf{b}}$ degree

(iii) Each infinite $\mathbf{b}$-computable tree in $2^{<\omega}$ has an infinite $\mathbf{a}^{-}$ computable path 
Proof. This may be proved by considering the case $\mathbf{b}=\mathbf{0}$ and relativizing. For $(\mathrm{i}) \rightarrow($ ii), assume $\mathbf{a}>>\mathbf{0}$ and consider the computable function $\psi^{*}$, where $\psi^{*}(n)=1$ if $P A \vdash \varphi_{n}$ and $\psi^{*}(n)=0$ if $P A \vdash \neg \varphi_{n}$. As we have remarked, each total extension of $\psi^{*}$ computes a completion of $P A$, so a is a $P A$ degree. The implication (ii) $\rightarrow$ (iii) follows at once from Scott's Theorem (Theorem 8.4). The proof of (iii) $\rightarrow$ (i) is implicit in the proof that there is a low degree $\mathbf{a}>>\mathbf{0}$ just after Definition 4.1.

8.5. Relativizing to models of $R C A_{0}$. We claim that Scott's Theorem, once formalized in second-order arithmetic, is provable in $R C A_{0}$. This is basically because Scott's Theorem is proved in an effective manner. (Details of similar theorems can be found in Simpson [1999].)

Let $S S$ be the assertion (formalized in second-order arithmetic) that every real belongs to some countable Scott set. Of course, one refers to countable Scott sets in this context by referring to the reals which uniformly enumerate them.

Lemma 8.18. SS is equivalent to Weak König's Lemma over $R C A_{0}$.

Proof. We argue in $R C A_{0}$. It is obvious that $S S$ implies Weak König's Lemma over $R C A_{0}$. Conversely, assume Weak König's Lemma. For any real $X$, there is an infinite tree $\operatorname{Tr}^{X}$ all of whose branches compute completions of $P A^{X}$ and hence, by Scott's Theorem relative to $X$, compute Scott sets containing $X$. By Weak König's Lemma, $\operatorname{Tr}^{X}$ has a branch, so there is a countable Scott set containing $X$ as an element.

Lemma 8.19. Every countable model of $R C A_{0}$ is an $\omega$-submodel of a countable model of $R C A_{0}+S S$.

Proof. This is immediate from Theorem 8.3 and the previous lemma.

We get a similar lemma by applying Lemma 8.7. It will be used in Section 11.

Lemma 8.20. For every countable topped model $\mathcal{M}$ of $R C A_{0}+I \Sigma_{3}$ and every real $X$ of $\mathcal{M}$, there is a topped model $\mathcal{M}^{\prime}$ of $R C A_{0}+I \Sigma_{3}$ and a real $Y$ of $\mathcal{M}^{\prime}$ such that, in $\mathcal{M}^{\prime}, Y$ codes a Scott set having $X$ as a member.

Proof. Use Lemma 8.7 to add a path $T$ though $\operatorname{Tr}^{X}$ to $\mathcal{M}$. Apply Lemma 5.3 to get a model $\mathcal{M}^{\prime}$ of $R C A_{0}$. Now $\mathcal{M}^{\prime}$ is a model of " $\mathcal{S}_{T}$ is a Scott set and $X \in \mathcal{S}_{T}$ " and $\mathcal{M}^{\prime}$ is topped by $T \oplus D$, where $\mathcal{M}$ is topped by $D$. 


\section{9. $\mathrm{COH}$}

9.1. Conservation theorems for $\mathrm{COH}$. The following theorem which is the analogue in second-order arithmetic of the existence of a low $_{2} \mathrm{r}$-cohesive set (or, more precisely, of an infinite $\mathrm{low}_{2}$ set not split by any set in a given uniformly computable sequence of sets).

Theorem 9.1. $R C A_{0}+C O H$ is $\Pi_{1}^{1}$-conservative over $R C A_{0}$.

The theorem follows from the next lemma, which is proved in Section 9.2 .

Lemma 9.2. Let $\mathcal{M}$ be any countable model of $R C A_{0}$ and let $\left(R_{i}\right)$ be a sequence of sets coded in $\mathcal{M}$. Then there is an $\vec{R}$-cohesive set $G$ such that $\mathcal{M}[G]$ is a model of $I \Sigma_{1}$.

Proof of Theorem 9.1 from Lemma 9.2. Let $\mathcal{M}$ be a model of $R C A_{0}$. Let $\left(R_{i}\right)$ be a sequence of sets which is coded in $\mathcal{M}$. Apply Lemma 9.2 to get $\mathcal{M}^{\prime}$. Then apply Lemma 6.7 to get a model $\mathcal{M}^{\prime \prime}$ of $R C A_{0}$. Iterate the process infinitely many times ensuring that for every such sequence of sets $R_{i}$ a $\vec{R}$-cohesive set $G$ is added. The resulting model is a model of $R C A_{0}+C O H$. Theorem 9.1 now follows from Lemma 6.6 and the following corollary to the argument just given.

Corollary 9.3. Every countable model of $R C A_{0}$ is an $\omega$-submodel of some countable model of $\mathrm{RCA}_{0}+\mathrm{COH}$.

In later sections, we will need some lemmas similar to Lemma 9.2 and Corollary 9.3 .

Lemma 9.4. Let $\mathcal{M}$ be any countable model of $R C A_{0}+I \Sigma_{2}+W K L$ and let $\left(R_{i}\right)$ be a sequence of sets coded in $\mathcal{M}$. There is an $\vec{R}$-cohesive set $G$ such that $M[G]$ satisfies $I \Sigma_{2}$.

Lemma 9.5. Every countable model of $R C A_{0}+I \Sigma_{2}$ is an $\omega$-submodel of some countable model of $W K L_{0}+I \Sigma_{2}+C O H$.

Proof of Lemma 9.5 from Lemma 9.4. Let $\mathcal{M}$ be a model of $R C A_{0}+I \Sigma_{2}$. Apply Lemma 8.6 to get a model $\mathcal{M}^{\prime}$ of $W K L_{0}+I \Sigma_{2}$ of which $\mathcal{M}$ is an $\omega$-submodel. Choose some $\left(R_{i}\right)$, a sequence of sets which is coded in $\mathcal{M}^{\prime}$. Apply Lemma 9.4 to get a $\vec{R}$-cohesive set $G$ which can be added to $\mathcal{M}^{\prime}$ while preserving $I \Sigma_{2}$. Then apply Lemma 6.7 to get a model $\mathcal{M}^{\prime \prime}$ of $R C A_{0}+I \Sigma_{2}$. Iterate the process infinitely many times ensuring that for every such sequence of sets $R_{i}$ a $\vec{R}$-cohesive set $G$ is added. The resulting model is a model of $W K L_{0}+I \Sigma_{2}+C O H$. 
Lemma 9.5 and Lemma 6.6 imply that $W K L_{0}+C O H+R C A_{0}+I \Sigma_{2}$ is $\Pi_{1}^{1}$-conservative over $R C A_{0}+I \Sigma_{2}$. But using Theorem 8.3 and Corollary 9.3 we can slightly improve this.

Lemma 9.6. Every countable model of $R C A_{0}$ is an $\omega$-submodel of some countable model of $\mathrm{WKL}_{0}+\mathrm{COH}$.

Proof. Let $\mathcal{M}$ be a model of $R C A_{0}$. Apply Theorem 8.3 to get a model $\mathcal{M}^{\prime}$ of $W K L_{0}$ of which $\mathcal{M}$ is an $\omega$-submodel. Apply Lemma 9.3 to get a model $\mathcal{M}^{\prime \prime}$ of $R C A_{0}+C O H$ of which $\mathcal{M}^{\prime}$ is an $\omega$-submodel. Iterate the process infinitely many times. Since $C O H$ and Weak König's Lemma are $\Pi_{2}^{1}$, the resulting model is a model of $W K L_{0}+C O H$.

By Lemma 9.6 and Lemma 6.6. $W K L_{0}+C O H$ is $\Pi_{1}^{1}$-conservative over $R C A_{0}$. We should point out that it is unclear if this conservation result or Lemma 9.6 implies Lemma 9.5. For related issues see Question 13.3 and Question 13.4 .

The next lemma will be useful in the proof of Theorem 11.2 .

Lemma 9.7. Assume $\mathcal{M}$ is a model of $R C A_{0}+I \Sigma_{3}$. Let $\left(R_{i}\right)$ be a sequence of sets in $\mathcal{M}$ coded by a set $C \in \mathcal{M}$. Furthermore assume $\mathcal{M}$ has a set $T$ which uniformly codes a Scott set $\mathcal{S}_{T}$ containing $C$. Then there is an $\vec{R}$-cohesive set $G$ such that $M[G]$ satisfies $I \Sigma_{3}$.

The proof of Lemma 9.2 can be found in Section 9.2, the proof of Lemma 9.4 can be found in Section 9.3 and the proof of Lemma 9.7 can be found in Section 9.4.

9.2. The proof of Lemma 9.2. Fix a model $\mathcal{M}=(\mathbb{X}, \mathcal{F},+, \times, 0,1,<)$ of $R C A_{0}$. We will call $\mathcal{M}$ the ground model. We will assume in this subsection that all numbers and sets (except for $G$ ) are in the ground model.

This argument will be modeled on the argument in Section 5.1. However, it will be simpler since we need concern ourselves only with $\Sigma_{1}^{0}$ formulas rather than $\Sigma_{2}^{0}$-formulas.

We will add an unbounded set $G$ such that for all $S \in \mathcal{F}$ either $G \subseteq^{*} S$ or $G \subseteq^{*} \bar{S}$ while preserving $I \Sigma_{1}$. So $G$ is cohesive for the sequence of all sets in $\mathcal{F}$ and could be said to be $\overrightarrow{\mathcal{M}}$-cohesive. This sequence is not coded in $\mathcal{M}$ and hence in reality we are proving a result stronger than that claimed by Lemma 9.2 .

We force over $\mathcal{M}$ using conditions $(D, L)$ where $D$ is $\mathcal{M}$-finite, $L$ is an $\mathcal{M}$-infinite set (in $\mathcal{F}$ ) and each element of $D$ is less than each element of $L$. We say $\left(D^{*}, L^{*}\right)$ extends $(D, L)$ iff $D \subseteq D^{*} \subset D \cup L$ and $L^{*} \subseteq L$. A set $G \subseteq \mathbb{X}$ satisfies a condition $(D, L)$ if $D \subseteq G \subseteq D \cup L$. A condition $(D, L)$ forces a $\Pi_{1}^{0}$ formula $\varphi(G)$ if for all $\mathcal{M}$-finite subsets $F$ 
of $L, \varphi(D \cup F)$. (This is the same as Definition 5.1, but in the context of $\mathcal{M}$.) In this case, $\varphi(G)$ holds for all sets $G$ satisfying $(D, L)$, since the failure of $\varphi(G)$ uses only $\mathcal{M}$-finitely much information about $G$. It is clear that if a condition $(D, L)$ fails to force a $\Pi_{1}^{0}$ formula $\varphi(G)$ then $(D, L)$ has an $\mathcal{M}$-finite extension $\left(D^{*}, L^{*}\right)$ which forces $\neg \varphi(G)$.

A condition $(D, L)$ extends a binary string $\tau$ if $\tau^{-1}(1) \subseteq D$ and $\tau^{-1}(0) \subseteq \mathbb{X}-(D \cup L)$. (This is equivalent to saying that every set which satisfies $(D, L)$ extends $\tau$.) For a condition $(D, L)$ and a $\Pi_{1}^{0}$ formula $(\forall \vec{x}) \theta(\vec{x}, G)$, where $\theta(\vec{x}, G)$ is $\Delta_{0}^{0}$, we say that $(D, L)$ forces $\neg(\forall \vec{x}) \theta(\vec{x}, G)$ if there is a tuple $\vec{w}$ of parameters from $\mathbb{X}$ and a binary string $\tau$ such that $(D, L)$ extends $\tau$ and $\tau$ forces $\neg \theta(\vec{w}, G)$. Here, forcing of $\Delta_{0}^{0}$ formulas by binary strings is defined recursively as indicated in Definition 5.1. Clearly, forcing implies truth for negations of $\Pi_{1}^{0}$ formulas. Also, it is clear that if a condition $(D, L)$ fails to force a $\Pi_{1}^{0}$ formula $\varphi(G)$ then $(D, L)$ has an $\mathcal{M}$-finite extension $\left(D^{*}, L^{*}\right)$ which forces $\neg \varphi(G)$.

Any generic $G$ for these conditions will meet dense sets to ensure that $G$ is not split by any set in $\mathcal{F}$. Suppose that some condition $(D, L)$ and some set $R \in \mathcal{F}$ is given. Then either $(D, L \cap R)$ or $(D, L-R)$ is a condition satisfied only by sets which are not split by $R$. Also, for each $n \in \mathcal{M}$ and each condition $(D, L)$ there is a condition $\left(D^{*}, L^{*}\right)$ extending $(D, L)$ such that $\left|D^{*}\right| \geq n$ (in the sense of $\mathcal{M}$ ), so that any sufficiently generic set $G$ is $\mathcal{M}[G]$-infinite. Hence any sufficiently generic set $G$ for these forcing conditions is an $\vec{M}$-cohesive set.

9.2.1. Preserving $I \Sigma_{1}$. For all $\varphi(x, G)$, a $\Pi_{1}^{0}$-formula with parameters from $\mathcal{M}$, we want to ensure either $(\forall x) \varphi(x, G)$ or for some $b, \neg \psi(b, G) \wedge(\forall x<b) \varphi(x, G)$. Hence we are ensuring that every nonempty set which is $\boldsymbol{\Pi}_{\mathbf{1}}^{\mathbf{0}, \mathbf{G}}$ over $\mathcal{M}$ (with parameters from $\mathcal{F} \cup\{G\}$ ) has a least element.

We show how to ensure this for a given $\Pi_{1}^{0}$ formula $\varphi(x, G)$ by extending a given condition $(D, L)$. Let $S=\{c \in \mathbb{X}:(D, L)$ does not force $\varphi(c, G)\}$. It is easily seen that $S$ is $\Sigma_{1}^{0}$ over $\mathcal{M}$. If $S=\emptyset,(D, L)$ forces $\varphi(a, G)$ for every $a \in \mathcal{M}$, so $(\forall x) \varphi(x, G)$ holds for every set $G$ which satisfies $(D, L)$. If $S \neq \emptyset, S$ has a least element $b$ by $I \Sigma_{1}$ in the ground model $\mathcal{M}$. Then $(D, L)$ forces $\psi(c, G))$ for each $c<b$ and so does each extension of $(D, L)$. As remarked above, there is an $\mathcal{M}$-finite extension $\left(D^{*}, L^{*}\right)$ of $(D, L)$ which forces $\neg \theta(b, G)$. Hence $b$ is the least element of $\mathbb{X}$ satisfying $\theta(x, G)$ for any set $G$ which satisfies $\left(D^{*}, L^{*}\right)$. 
9.2.2. Putting it all together. Let $\left(R_{s}\right)_{s \in N}$ be a listing of the various requirements discussed in the previous section to ensure that $G$ is $\vec{M}-$ cohesive and that $M[G]$ satisfies $I \Sigma_{1}$. Construct a sequence of conditions $\left(D_{s}, L_{s}\right)$ with $\left(D_{s+1}, L_{s+1}\right)$ extending $\left(D_{s}, L_{s}\right)$ and chosen so that every set satisfying $\left(D_{s+1}, L_{s+1}\right)$ satisfies the requirement $R_{s}$. Let $G=\cup_{s} D_{s}$. Then $G$ satisfies all the requirements $R_{s}$.

9.3. The proof of Lemma 9.4. This argument will be based on the arguments presented in Sections 5.2 and 9.2. Fix a model $\mathcal{M}=(\mathbb{X}, \mathcal{S},+, \times, 0,1,<)$ of $W K L_{0}+I \Sigma_{2}$. We will call $\mathcal{M}$ the ground model.

We will add a set $G$ such that for all $S \in \mathcal{S}$ either $G \subseteq^{*} S$ or $G \subseteq \subseteq^{*} \bar{S}$ while preserving $I \Sigma_{2}$. So $G$ is $\overrightarrow{\mathcal{M}}$-cohesive. Hence in reality we are proving a result stronger than that claimed by Lemma 9.4.

Except for the sets $G$ and $Z$, we will assume in this subsection that all numbers and sets mentioned are in the ground model.

We force over $\mathcal{M}$ using the conditions $(D, L)$ where $D$ is $\mathcal{M}$-finite, $L$ is an $\mathcal{M}$-infinite set in $\mathcal{S}$ and each element of $D$ is less than each element of $L$. Let $\varphi(G)$ be $\Pi_{1}^{0}$. We say $(D, L)$ forces $\varphi(G)$ if for all $\mathcal{M}$-finite subsets $F$ of $L, \varphi(D \cup F)$ holds in $\mathcal{M}$. We say $(D, L)$ forces $(\exists \vec{x}) \varphi(\vec{x}, G)$ if for some $\vec{w},(D, L)$ forces $\varphi(\vec{w}, G)$.

9.3.1. Preserving $I \Sigma_{2}$. For all $\theta(x, G)$, a $\Sigma_{2}^{0}$-formula with parameters from $\mathcal{M}$, and all numbers $a$ in $\mathcal{M}$, we want to ensure either $(\forall x \leq a) \theta(x, G)$ or for some $b \leq a, \neg \theta(b, G) \wedge(\forall x<b) \theta(x, G)$. Hence we are ensuring that every nonempty set which is $\boldsymbol{\Pi}_{\mathbf{2}}^{\mathbf{0}}$ over $\mathcal{M}[G]$ has a least element.

Definition 9.8. Let $(D, L)$ be a condition and let $S=\left\{\left(\exists \overrightarrow{x_{1}}\right) \varphi_{1}\left(\overrightarrow{x_{1}}, G\right)\right.$, $\left.\ldots,\left(\exists \overrightarrow{x_{k}}\right) \varphi_{k}\left(\overrightarrow{x_{k}}, G\right)\right\}$ be an $\mathcal{M}$-finite set of $\Sigma_{2}^{0}$ formulas, with each formula $\varphi_{i}\left(\vec{x}_{i}, G\right)$ a $\Pi_{1}^{0}$ formula.

We define what it means for $(D, L)$ to be $S-$ small as in Definition 5.2 except that, of course, this definition is now interpreted in $\mathcal{M}$. More precisely, $(D, L)$ is $S$-small if there exist a number $n$ of $\mathcal{M}$ and sequences $\left(\vec{w}_{i}: i<n\right)$ and $\left(D_{i}, L_{i}, k_{i}: i<n\right)$ coded in $\mathcal{M}$ such that the $L_{i}$ 's are a partition of $L$; for each $i, D \subseteq D_{i} \subset D \cup L$; for each $i$, every element of $D_{i}$ is less than every element of $L_{i}$; and for each $i$, either $L_{i}$ has no element greater than $\max \left(\vec{w}_{i}\right)$ or $\left(D_{i}, L_{i}\right)$ forces $\varphi_{k_{i}}\left(\vec{w}_{i}, G\right)$. The condition $(D, L)$ is $S$-large if it is not $S$-small.

The following lemma gives the basic combinatorial property we need of smallness. Its analogue in the context of Section 3 was obvious. However, a proof is needed now since we must show that the appropriate sequences are $\mathcal{M}$-finite. 
Lemma 9.9. Suppose $S$ is an $\mathcal{M}$-finite set of $\Sigma_{2}^{0}$ formulas, $k$ is a number of $\mathcal{M}$ and that $\left(\left(D_{i}, L_{i}\right): i<k\right)$ is an $\mathcal{M}$-finite sequence of $S$-small conditions in the sense of $\mathcal{M}$. Let $D$ be an $\mathcal{M}$-finite set such that $D \subseteq D_{i}$ for each $i<k$, and let $L=\cup_{i<k} L_{i}$. Then $(D, L)$ is an $S-$ small condition.

Proof. The idea is simply to combine the witnesses that each $\left(D_{i}, L_{i}\right)$ is $S$-small to produce a witness that $(D, L)$ is $S$-small. However, it must be shown that this operation can be carried out in $\mathcal{M}$.

First note that the definition of $S$-smallness for $\left(D^{*}, L^{*}\right)$ can be written in the form $(\exists w)(\exists X) P\left(w, X, D^{*}, L^{*}\right)$ where $P$ is a $\Pi_{1}^{0}$ formula. This can be done by contraction of the number and set quantifiers in the definition of smallness. (The number of set quantifiers was variable but may be coded into $w$.) Thus $\mathcal{M}$ satisfies the formula $(\forall i<k)(\exists w)(\exists X) P\left(w, X, D_{i}, L_{i}\right)$. The formula $(\exists X) P\left(w, X, D_{i}, L_{i}\right)$ is $\Pi_{1}^{0}$ since $\mathcal{M}$ is a model of Weak König's Lemma and hence this statement can be rewritten as the assertion that a certain binary-branching tree (whose paths are the possible $X$ 's) which is coded by a real in $\mathcal{M}$ is infinite. Thus, since $\mathcal{M}$ satisfies $I \Sigma_{2}$ and hence $B \Sigma_{2}$ it follows that $\mathcal{M}$ satisfies $(\exists b)(\forall i<k)(\exists w<b)(\exists X) P\left(w, X, D_{i}, L_{i}\right)$. Fix such a $b$.

Now use that $\mathcal{M}$ satisfies $I \Sigma_{2}$ to show that $\mathcal{M}$ satisfies the formula $(\exists X)(\forall i<a)(\exists w<b) P\left(w,(X)_{i}, D_{i}, L_{i}\right)$ for each $a \leq k$. (This is an induction on $a$. It uses that $(\forall i<a)(\exists w<b) P\left(w,(X)_{i}, D_{i}, L_{i}\right)$ is equivalent to a $\Pi_{1}^{0}$ formula by standard quantifier manipulations, so that $(\exists X)(\forall i<a)(\exists w<b) P\left(w,(X)_{i}, D_{i}, L_{i}\right)$ is equivalent to a $\Sigma_{2}^{0}$ formula. The base step is trivial, and the induction step follows from the hypothesis that $(\forall i<k)(\exists w)(\exists X) P\left(w, X, D_{i}, L_{i}\right)$.) Now, fix a real $X$ of $\mathcal{M}$ such that $\mathcal{M}$ satisfies $(\forall i<k)(\exists w<b) P\left(w,(X)_{i}, D_{i}, L_{i}\right)$. Then there is a number $z$ of $\mathcal{M}$ such that $\mathcal{M}$ satisfies $(\forall i<k) P\left((z)_{i},(X)_{i}, D_{i}, L_{i}\right)$. (Again using $I \Sigma_{2}$.) Then from $X$ and $z$ one can decode an $\mathcal{M}$-finite sequence of sets and numbers which witnesses that $(D, L)$ is $S$-small.

As in Section 5.1.2, we will restrict ourselves to $S$-large conditions for various $S$ as the construction proceeds. Thus we consider how to extend a given $S$-large condition to an $S$-large condition which forces a given requirement to be satisfied. We first do this for the requirements used for $I \Sigma_{2}$.

Lemma 9.10. Suppose that $(D, L)$ is a condition and $S$ is a finite set of $\Sigma_{2}^{0}$ formulas and that $(D, L)$ is $S$-large. Let $(\exists x) \theta(x, y, G)$ be a $\Sigma_{2}^{0}$ formula, where $\theta(x, y, G)$ is a $\Pi_{1}^{0}$ formula, and let a be a number in $\mathcal{M}$. Then there is an $S$-large condition $\left(D^{*}, L^{*}\right)$ extending $(D, L)$ such that either 
- $\left(D^{*}, L^{*}\right)$ forces $(\exists x) \theta(x, b, G)$ for all $b \leq a$, or

- There exists $b \leq a$ such that $\left(D^{*}, L^{*}\right)$ forces $(\exists x) \theta(x, c, G)$ for all $c<b$ and $\left(D^{*}, L^{*}\right)$ is $(S \cup\{(\exists x) \theta(x, b, G)\})$-large.

Proof. Let $R$ be the set of $b \leq a$ such that there exist a number $k$, sets $L_{0}, L_{1}, \ldots, L_{k}$ with $L=\sqcup_{i \leq k} L_{i}, \mathcal{M}$-finite sets $F_{0}, F_{1}, \ldots F_{k}$ with $D \subseteq F_{i} \subseteq D \cup L$, and each element of $F_{i}$ less than each element of $L_{i}$, formulas $\varphi_{0}(G), \ldots, \varphi_{k}(G)$ and numbers $w_{0}, \ldots, w_{k}$ and such that for each $i \leq k$ either:

(1) $\varphi_{i}(G)$ is a $\Pi_{1}^{0}$-instance of a formula in $S$ and $\left(F_{i}, L_{i}\right)$ forces $\varphi_{i}(G)$

(2) $w_{i}$ codes an $\mathcal{M}$-finite sequence $w_{i}^{0}, w_{i}^{1}, \ldots, w_{i}^{b}$ such that $\left(F_{i}, L_{i}\right)$ forces $\theta\left(w_{i}^{j}, j, G\right)$ for each $j \leq b$, or

(3) Every element of $L_{i}$ is less than $w_{i}$.

Note that the above definition is to be interpreted in $\mathcal{M}$. In particular, $k \in M,\left(L_{0}, \ldots, L_{k}\right)$ is coded by a set of $M$, etc. The set $R$ is $\boldsymbol{\Sigma}_{\mathbf{2}}^{\mathbf{0}}$ over $\mathcal{M}$. To see this, note that the definition of $R$ could be phrased as $(\exists k)(\exists \vec{F})(\exists \vec{w})(\exists \vec{L}) P(k, \vec{F}, \vec{w}, \vec{L})$, where $P$ is a $\Pi_{1}^{0}$ formula. The formula $(\exists \vec{L}) P$ is then also equivalent to a $\Pi_{1}^{0}$ formula over $\mathcal{M}$, since by Weak König's Lemma it is equivalent to the assertion that a certain tree (whose paths are the possible $\vec{L}$ ) which is a set in $\mathcal{M}$ contains strings of every length. Thus, $R$ can be defined over $\mathcal{M}$ by a $\Sigma_{2}^{0}$ formula.

Suppose first that $a \in R$, and consider the corresponding $k, L_{0}, \ldots, L_{k}$, $F_{0}, \ldots, F_{k}, w_{0}, \ldots, w_{k}$, and $\varphi_{0}, \ldots, \varphi_{k}$. Note by Lemma 9.9 that for some $i \leq k$, the pair $\left(F_{i}, L_{i}\right)$ is an $S$-large condition, since $(D, L)$ is $S$-large. For such an $i,\left(F_{i}, L_{i}\right)$ is an $S$-large condition which extends $(D, L)$ and forces $(\exists x) \theta(x, b, G)$ for each $b \leq a$, so the conclusion of the lemma holds with $\left(D^{*}, L^{*}\right)=\left(F_{i}, L_{i}\right)$.

Now suppose that $a \notin R$. Since $\mathcal{M}$ satisfies $I \Sigma_{2}$, there is a least number $b \notin R$, and $b \leq a$. First suppose that $b=0$. Then $(D, L)$ is $(S \cup\{(\exists x) \theta(x, 0, G)\})$-large, so the conclusion of the lemma holds with $\left(D^{*}, L^{*}\right)=(D, L)$. Now assume that $b>0$, and choose $k, L_{0}, \ldots, L_{k}, \ldots$ which witness that $b-1 \in R$. We claim that $\left(F_{i}, L_{i}\right)$ is $(S \cup\{(\exists x) \theta(x, b, G)\})$-large for some $i \leq k$. Once the claim is proved, it follows that the conclusion of the lemma holds with $\left(D^{*}, L^{*}\right)=\left(D_{i}, L_{i}\right)$, since $\left(D_{i}, L_{i}\right)$ forces $(\exists x) \theta(x, c, G)$ for each $c<b$, as in the case where $a \in R$.

To prove the claim, assume for a contradiction that $\left(F_{i}, L_{i}\right)$ is $(S \cup\{(\exists x) \theta(x, b, G)\})$-small for all $i \leq k$. Choose corresponding witnesses $L_{i, j}, j \leq k_{i}$, etc. Altogether, there are only $\mathcal{M}$-finitely many sets 
$L_{i, j}$, and this $\mathcal{M}$-finite collection of sets and the other corresponding witnesses show that $b \in R$. For the moment, we argue very informally.

We will focus on those $i$ where $\left(F_{i}, L_{i}\right)$ is $S$-large and $L_{i}$ is $\mathcal{M}$-infinite. Fix such an $i$. Then $\left(F_{i}, L_{i}\right)$ forces $(\exists x) \theta(x, c, G)$ for each $c<b$, as these pairs are witnessing that $b-1 \in R$. It follows then that the stronger condition $\left(D_{i, j}, L_{i, j}\right)$ forces $(\exists x) \theta(x, c, G)$ for each $c<b$. By our choice of $i$ and the fact that $\left(F_{i}, L_{i}\right)$ is $(S \cup\{(\exists x) \theta(x, b, G)\})$-small, there must be some $j$ such that $\left(D_{i, j}, L_{i, j}\right)$ forces a $\Pi_{1}^{0}$-instance of $(\exists x) \theta(x, b, G)$. Fix any such $j$. $\left(D_{i, j}, L_{i, j}\right)$ forces $(\exists x) \theta(x, c, G)$ for each $c \leq b$. Hence, $b \in R$, which gives a contradiction. (This argument may be formalized in a manner similar to the proof of Lemma 9.9.)

As in our previous constructions, when we restrict ourselves to $S$ large conditions, our intention is to make every formula in $S$ false in $\mathcal{M}[G]$. The following lemma ensures that this is possible.

Lemma 9.11. Let $S$ be a finite set of $\Sigma_{2}^{0}$ formulas, and let $(D, L)$ be an $S$-large condition. Suppose that $\theta(G)$ is a $\Pi_{1}^{0}$-instance of a formula in $S$. Then there is an $S$-large condition $\left(D^{*}, L^{*}\right)$ which extends $(D, L)$ and forces $\neg \theta(G)$.

Proof. The proof is entirely analogous to that of Lemma 5.3 .

9.3.2. Putting it all together. Let $R \in \mathcal{S}$. If $(D, L)$ is $S$-large then one of $(D, L \cap R)$ or $(D, L \cap \bar{R})$ must be $S$-large (otherwise $(D, L)$ is $S$-small).

Let $\left\{\left\langle\theta_{i}(x, G), a_{i}\right\rangle\right\}$ be a listing of all pairs where $\theta_{i}(x, G)$ is a $\Sigma_{2}^{0}$ formula with parameters from $\mathcal{M}$ and $a_{i}$ is a number in $\mathcal{M}$. Let $\left\{R_{i}\right\}$ be a listing of reals in $\mathcal{M}$, and let $\delta_{s}(G)$ be a listing of the $\Pi_{1}^{0}$ formulas with parameters from $G$ such that each such formula occurs infinitely often in the list.

We will construct conditions $\left(D_{s}, L_{s}\right)$ such that $G=\cup_{s}\left\{D_{s}\right\}$ is an $\overrightarrow{\mathcal{M}}$-cohesive set and preserves $I \Sigma_{2}$. In addition, we construct finite sets $\left\{S_{s}\right\}$ of $\Sigma_{2}^{0}$-formulas. During and after stage $s$, we will commit ourselves to working with $S_{s}$-large conditions and ensuring $(\forall \vec{x}) \neg \varphi(\vec{x}, G)$ for all $(\exists \vec{x}) \varphi(\vec{x}, G) \in S_{s}$. Initially, let $\left(D_{-1}, L_{-1}\right)=(\emptyset, \tilde{L})$, (let $\left.\tilde{L}=\mathbb{X}\right)$ and $S_{-1}=\emptyset$. We can assume inductively that $\left(D_{s-1}, L_{s-1}\right)$ is $S_{s-1}$-large.

Stage s: Use Lemma 9.10 to find an $S_{s-1}$-large condition $\left(D^{*}, L^{*}\right)$ extending $\left(D_{s-1}, L_{s-1}\right)$ such that either $\left(D^{*}, L^{*}\right)$ forces $\left(\theta_{s}(b, G)\right)$ for all $b \leq a_{s}$ or for some $b \leq a_{s},\left(D^{*}, L^{*}\right)$ forces $\left(\theta_{s}(c, G)\right)$ for all $c<b$ and $\left(D^{*}, L^{*}\right)$ is $S_{s}$-large, where $S_{s}=S_{s-1} \cup\left\{\theta_{s}(b, G)\right\}$ in the latter case, and $S_{s}=S_{s-1}$ otherwise.

If $\delta_{s}(G)$ is a $\Pi_{1}^{0}$-instance of some formula in $S_{s}$, let $\left(D^{* *}, L^{* *}\right)$ be an $S_{s}$-large condition which extends $\left(D^{*}, L^{*}\right)$ and forces $\neg \delta_{s}(G)$. (Such a 
condition exists by Lemma 9.11.) Otherwise, let $\left(D^{* *}, L^{* *}\right)=\left(D^{*}, L^{*}\right)$. Furthermore, we may require that, in the sense of $\mathcal{M}$, the cardinality of $D^{* *}$ is $\geq a_{s}$.

Finally, let $\left(D_{s}, L_{s}\right)=\left(D^{* *}, L^{* *} \cap R_{s}\right)$ if $\left(D^{* *}, L^{* *} \cap R_{s}\right)$ is $S_{s}$-large; otherwise let $\left(D_{s}, L_{s}\right)=\left(D^{* *}, L^{* *} \cap \overline{R_{s}}\right)$.

Let $G=\cup_{s} D_{s}$. As forcing implies truth for the notions of forcing considered in this proof, it is easily seen that the model $M[G]$ obtained by adjoining $G$ to the reals of $\mathcal{M}$ has the desired properties.

9.4. The proof of Lemma 9.7. The proof is similar to that in Section 9.3 , although the class of forcing conditions is now chosen so that it will be possible to quantify over conditions using number quantifiers. Fix a model $\mathcal{M},\left\{R_{i}\right\}, C, T$ and $S_{T}$ as in Lemma 9.7. We will add an unbounded set $G$ such that for all $i$ either $G \cap R_{i}$ or $G \cap \overline{R_{i}}$ is bounded in $\mathcal{M}$ while preserving $I \Sigma_{3}$. So $G$ is $\vec{R}$-cohesive.

Except for the sets $G$, we will assume in this subsection that all numbers and sets mentioned are in the ground model. We force over $\mathcal{M}$ using the conditions $(D, L)$ where $D$ is $\mathcal{M}$-finite, $L \in \mathcal{S}_{T}, L$ is an $\mathcal{M}$-infinite set, and every element of $D$ is less than every element of $L$. This will help us to quantify over conditions (see Lemma 9.12). We define what it means for a condition $(D, L)$ to be $S$-large as in Section 9.3 .

9.4.1. Preserving $I \Sigma_{3}$. For all $\gamma(x, G)$, a $\Sigma_{3}^{0}$-formula with parameters from $\mathcal{M}$, and all numbers $a$ in $\mathcal{M}$, we want to ensure either $(\forall x \leq a)(\gamma(x, G))$ or for some $b \leq a, \neg \gamma(b, G) \wedge(\forall x<b)(\gamma(x, G))$. Hence we are ensuring that every nonempty $\Pi_{3}^{0, G}$-definable set (with parameters in $\mathcal{M}$ ) has a least element.

Suppose we are are given an $S$-large condition $(D, L)$, where $S$ is an $\mathcal{M}$-finite set of $\Sigma_{2}^{0}$ formulas. (We can no longer assume that $S$ is actually finite, as we did in Sections 9.2.1 and 9.3.1; see the next lemma.) If we wish to ensure that a $\Sigma_{2}^{0}$ formula $\delta(G)$ is false, we know that it is possible to do this if $(D, L)$ is $(S \cup\{\delta(G)\})$-large, by committing to work with $(S \cup\{\delta(G)\})$-large conditions from now on.

Lemma 9.12. Let $S$ be an $\mathcal{M}$-finite set of $\Sigma_{2}^{0}$ formulas and let $(D, L)$ be an $S$-large condition. Suppose that $\delta(x, y, G)$ is a $\Sigma_{2}^{0}$ formula. Let $C$ be the set of $c$ such that for some $\mathcal{M}$-finite set $S^{*} \supseteq S$ of $\Sigma_{2}^{0}$ formulas and some condition $\left(D^{*}, L^{*}\right)$ extending $(D, L),\left(D^{*}, L^{*}\right)$ is $S^{*}$-large and $(\forall b<c)(\exists d)\left[\delta(d, b, G) \in S^{*}\right]$. Then $C$ is $\mathbf{\Sigma}_{\mathbf{3}}^{\mathbf{0}}$ over $\mathcal{M}$.

Proof. There is a $\Pi_{2}^{0}$ formula $\lambda\left(S^{*}, D^{*}, L^{*}\right)$ such that whenever $S^{*}$ is a (code for) an $M$-finite set of $\Sigma_{2}^{0}$ formulas, $D^{*}$ is an $\mathcal{M}$-finite set, 
and $L^{*} \in \mathcal{S}_{T}$, then $\lambda\left(S^{*}, D^{*}, L^{*}\right)$ holds in $\mathcal{M}$ iff $\left(D^{*}, L^{*}\right)$ is an $S^{*-}$ large condition. This is proved as usual (for example, see the proof of Lemma 9.9), except rather than using that $\mathcal{M}$ satisfies Weak König's Lemma we use the fact that " $S_{T}$ is a Scott Set" in true in $\mathcal{M}$. In the definition of $C$, the quantifier over $L^{*}$ can be replaced by a number quantifier, using a parameter for $T$. The rest is routine quantifier counting.

It is easily seen that the $\Sigma_{3}^{0}$ formula $\gamma(y, G)$ is equivalent over $\mathcal{M}$ to a formula of the form $(\exists x) \neg \delta(x, y, G)$, where $\delta(x, y, G)$ is $\Sigma_{2}^{0}$. Fix a condition $(D, L)$ and a $\mathcal{M}$-finite set $S$ where $(D, L)$ is $S$-large. Define $C$ as in Lemma 9.12 .

Consider now the case where $a+1 \in C$. Then there is an extension $\left(D^{*}, L^{*}\right)$ of $(D, L)$ and an $\mathcal{M}$-finite set $S^{*}$ of $\Sigma_{2}^{0}$ formulas containing $S$ such that for all $c \leq a$, there is a $d, \delta(d, c, G) \in S^{*}$ and $\left(D^{*}, L^{*}\right)$ is $S^{*}$-large. Hence if we continue to work with $S^{*}$-large conditions we will ensure as usual that for all $c \leq a$ there exists $d$ such that $M[G]$ satisfies $\neg \delta(d, c, G)$. It then follows that $M[G]$ satisfies $\gamma(c, G)$, for all $c \leq a$.

Suppose now that $a+1 \notin C$. Then, by $I \Sigma_{3}$ in $\mathcal{M}$ and Lemma 9.12 , there is a least number $b$ such that $b \notin C$, and clearly, $b \leq a+1$. Since $(D, L)$ is $S$-large, $b \neq 0$. Let $S^{*}, D^{*}, L^{*}$ witness that $b-1 \in C$. Then by extending to $\left(D^{*}, L^{*}\right)$ and committing to work with $S^{*}$-large conditions from now on, we can ensure that $M[G]$ satisfies $\gamma(c, G)$ for all $c<b-1$. Furthermore for all conditions $(\widehat{D}, \widehat{L})$ extending $\left(D^{*}, L^{*}\right)$ and for all $d$, if $(\widehat{D}, \widehat{L})$ is $S^{*}$-large then $(\widehat{D}, \widehat{L})$ is $\left(S^{*} \cup\{\delta(d, b-1, G)\}\right)-$ small. Hence at future stages we can extend our conditions to force $\delta(d, b-1, G)$ for all $d$, and thus ensure that $M[G]$ satisfies $\neg \gamma(b-1, G)$. Thus, $b-1 \leq a$, and $b-1$ is the least $c$ such that $\gamma(c, G)$ is false in $M[G]$.

9.4.2. Putting it all together. One constructs an ascending chain $\left(D_{s}, L_{s}\right)$ of forcing conditions and $\mathcal{M}$-finite sets $S_{s}$ and takes $G=\cup_{s} D_{s}$, using the results of the previous subsection to ensure that $G$ is $\vec{R}$ cohesive and $M[G]$ satisfies $I \Sigma_{3}$. We omit the details, which are analogous to those of Section 9.3.2.

9.5. Independence. We show that $\mathrm{COH}$ and Weak König's Lemma are independent over $R C A_{0}$. We build an $\omega$-model of $C O H$ where Weak König's Lemma fails and also an $\omega$-model of Weak König's Lemma where $\mathrm{COH}$ fails. There has already been some work in this direction; Hirst [1987, Theorem 6.10] showed that there is an $\omega$-model of Weak König's Lemma where $R T_{2}^{2}$ fails. 
Theorem 9.13. COH and Weak König's Lemma are independent over $R C A_{0}$.

The theorem follows from the next two lemmas.

Lemma 9.14. There is $\omega$-model of $W K L_{0}$ which is not a model of $\mathrm{COH}$.

Proof. By the Low Basis Theorem, there is a low complete extension $T$ of Peano arithmetic. Let $\mathcal{S}$ be the family of all sets binumerable in $T$, so that $\mathcal{S}$ is a Scott set and hence an $\omega$-model of $W K L_{0}$. As all computable sets are in $\mathcal{S}, \mathcal{S}$ contains a set which uniformly codes all primitive recursive sets. Thus, if $\mathcal{S}$ were a model of $C O H, \mathcal{S}$ would contain a $\mathrm{p}$-cohesive set, i.e. an infinite set not split by any primitive recursive set. However, no p-cohesive set is low (see Theorem 12.4 or Jockusch and Stephan [1993, Theorem 2.1]). Hence $\mathcal{S}$ is not a model of $\mathrm{COH}$.

Lemma 9.15. There is an $\omega$-model of $R C A_{0}+C O H$ which is not a model of Weak König's Lemma.

Lemma 9.15 is a consequence of the following lemma.

Lemma 9.16. Fix a real $A$. Suppose that $T$ is an infinite binary branching computable tree such that none of its infinite paths are computable from $A$. Finally, suppose that the sets $R_{0}, R_{1}, \ldots$ are each computable from $A$. Then there is an $\vec{R}$-cohesive set $G$ which does not compute any paths through $T$.

Proof. The requirements to be met are the following:

$$
(\exists x)\left[\{e\}^{G}(x) \uparrow \text { or }\{e\}^{G}\lceil x \notin T]\right.
$$

$$
|G| \geq e
$$

$$
G \subseteq^{*} R_{e} \text { or } G \subseteq \subseteq^{*} \overline{R_{e}}
$$

These will be met using forcing with conditions $(D, L)$, which are as in our previous arguments except that now $L$ is an infinite $A$ computable set (and need not be low). As before, it suffices to show that for any requirement $S_{s}$ and any condition $(D, L)$, there is a condition $\left(D^{\prime}, L^{\prime}\right)$ extending $(D, L)$ such that every set satisfying $\left(D^{\prime}, L^{\prime}\right)$ satisfies the requirement $S_{s}$. This is clear if $s$ is of the form $3 e+1$ or $3 e+2$, so assume that $s=3 e$. Also assume for a contradiction that a condition $(D, L)$ is given such that no such $\left(D^{\prime}, L^{\prime}\right)$ exists for $s=3 e$. We now obtain a contradiction by constructing an $A$-computable path $f$ through $T$. To do this we recursively define an infinite sequence 
of conditions $\left(D_{0}, L_{0}\right),\left(D_{1}, L_{1}\right), \ldots$ such that $\left(D_{0}, L_{0}\right)=(D, L)$ and $\left(D_{i+1}, L_{i+1}\right)$ is a finite extension of $\left(D_{i}, L_{i}\right)$ for each $i$. We also define an infinite sequence of strings $\tau_{0}, \tau_{1}, \ldots$ such that $\left|\tau_{i}\right| \geq i,\{e\}^{G}$ extends $\tau_{i}$ for all $G$ satisfying $\left(D_{i}, L_{i}\right)$, and $\tau_{i+1}$ extends $\tau_{i}$ for all $i$. It follows that $\tau_{i} \in T$, since otherwise $\left(D_{i}, L_{i}\right)$ would be a condition $\left(D^{\prime}, L^{\prime}\right)$ as above. Hence $f \in[T]$ where $f=\cup_{i} \tau_{i}$. Furthermore, $D_{i}, L_{i}$ and $\tau_{i}$ are $A$-computable, uniformly in $i$, so that $f$ is an $A$-computable path through $T$, yielding the desired contradiction.

To start the construction, let $\left(D_{0}, L_{0}\right)=(D, L)$, and let $\tau_{0}$ be the empty string. Now suppose that $D_{i}, L_{i}$, and $\tau_{i}$ have been constructed. There is a set $G$ satisfying $\left(D_{i}, L_{i}\right)$ with $\{e\}^{G}(i) \downarrow$, since otherwise $\left(D_{i}, L_{i}\right)$ would serve as $\left(D^{\prime}, L^{\prime}\right)$. Hence, by a standard construction, there is a finite extension $\left(D_{i+1}, L_{i+1}\right)$ of $\left(D_{i}, L_{i}\right)$ and a value $y_{i}$ such that $\{e\}^{G}(i)=y_{i}$ for all $G$ satisfying $\left(D_{i+1}, L_{i+1}\right)$. Furthermore, $D_{i+1}, L_{i+1}$, and $y_{i}$ may be found by an $A$-effective search. Let $\tau_{i+1}=\tau_{i}^{\frown} y_{i}$. This clearly works.

Proof of Lemma 9.15 from Lemma 9.16. Fix an infinite computable tree $T$ with no computable paths. (It is easy to see such a tree exists. For example, if $B_{0}$ and $B_{1}$ are disjoint computably inseparable computably enumerable sets. Then family of sets which separate $B_{0}$ and $B_{1}$ forms a nonempty $\Pi_{1}^{0}$ class in $2^{\omega}$ with no computable paths.) By iterating Lemma 9.16 it is possible to build an $\omega$-model of $R C A_{0}+C O H$ which does not contain any infinite path through $T$. To carry this out, let $H_{0}=\emptyset$. Assume inductively that $H_{t}$ is defined for $t \leq s$ and that $H_{s}$ does not compute an infinite path through $T$. Let $s=\langle e, k\rangle$, where $k<s$. If $\{e\}^{H_{k}}$ is not a characteristic function, let $H_{s+1}=H_{s}$. If $\{e\}^{H_{k}}$ is the characteristic function of a set $R$, let $R_{i}=(R)_{i}$, and let $G$ be as in Lemma 9.16, and let $H_{s+1}=H_{s} \oplus G$. It is clear by induction on $s$ that $H_{s}$ does not compute an infinite path through $T$. Thus, if $\mathcal{S}$ is the family of all sets computable from some $H_{s}$, then $\mathcal{S}$ is the desired $\omega$-model of $R C A_{0}+C O H+\neg$ Weak König's Lemma .

\section{Two COLORS}

The goal of this section is to obtain the following result.

Theorem 10.1. Every countable model of $R C A_{0}+I \Sigma_{2}$ is an $\omega-$ submodel of some countable model of $W K L_{0}+I \Sigma_{2}+R T_{2}^{2}$.

By Theorem 6.6, the above theorem immediately yields the following consequence.

Theorem 10.2. $R T_{2}^{2}+R C A_{0}+I \Sigma_{2}$ is $\Pi_{1}^{1}$-conservative over $R C A_{0}+I \Sigma_{2}$. 
In fact, it implies that $W K L_{0}+I \Sigma_{2}+R T_{2}^{2}$ is $\Pi_{1}^{1}$-conservative over $R C A_{0}+I \Sigma_{2}$. By a result of Slaman (Theorem 2.9), Theorem 10.1 fails for models of $R C A_{0}$, i.e. there is a countable model of $R C A_{0}$ which is not an $\omega$-submodel of any model of $R C A_{0}+R T_{2}^{2}$.

Theorem 10.1 follows from Lemma 9.5 and the following result.

Theorem 10.3. Every countable model of $R C A_{0}+I \Sigma_{2}$ is an $\omega-$ submodel of some countable model of $W K L_{0}+I \Sigma_{2}+S R T_{2}^{2}$.

Proof of Theorem 10.1. Start with any countable model $\mathcal{M}$ of $R C A_{0}+I \Sigma_{2}$. By Lemma 9.5, $\mathcal{M}$ is an $\omega$-submodel of a countable model $\mathcal{M}_{1}$ of $R C A_{0}+I \Sigma_{2}+C O H$. By Theorem $10.3 \mathcal{M}_{1}$ is an $\omega$-submodel of a countable model $\mathcal{M}_{2}$ of $W K L_{0}+I \Sigma_{2}+S R T_{2}^{2}$. Iterate to get a countable $\omega$-chain of countable models whose union is a model of $W K L_{0}+I \Sigma_{2}+S R T_{2}^{2}+C O H$. By Lemma 7.11, $R T_{2}^{2}$ follows from $R C A_{0}+C O H+S R T_{2}^{2}$.

The following result is used to prove Theorem 10.3

Lemma 10.4. Let $\mathcal{M}$ be a countable model of $W K L_{0}+I \Sigma_{2}$. Let $f(x, s)$ be a function coded in $\mathcal{M}$ such that for all $x$ and $s, f(x, s)<2$, and for all $x, \lim _{s} f(x, s)$ exists. It is possible to add an unbounded set $G$ while preserving $I \Sigma_{2}$ such that for some $j<2$, for all $x \in G, \lim _{s} f(x, s)=j$.

Proof of Theorem 10.3. Start with any countable model $\mathcal{M}$ of $R C A_{0}+I \Sigma_{2}$. By Lemma 8.6 it is an $\omega$-submodel of a countable model $\mathcal{M}^{\prime}$ of $W K L_{0}+I \Sigma_{2}$. Given $f$ coded in $\mathcal{M}^{\prime}$ as in Lemma 10.4, use Lemma 10.4 to form a new model $\mathcal{M}^{\prime \prime}$ by adding the set $G$ while preserving $I \Sigma_{2}$. By Lemma 6.7 $\mathcal{M}^{\prime \prime}$ is an $\omega$-submodel of a countable model of $R C A_{0}+I \Sigma_{2}$. Iterate over all such functions and take the union of a chain to get a model $\mathcal{M}^{*}$ of $W K L_{0}+I \Sigma_{2}+D_{2}^{2}$. By Lemma 7.10 , over $R C A_{0}, D_{2}^{2}$ is equivalent to $S R T_{2}^{2}$. So $\mathcal{M}^{*}$ is a model of $W K L_{0}+I \Sigma_{2}+S R T_{2}^{2}$.

The following theorem is a slight improvement of the above mentioned result of Slaman (Theorem 2.9) and it shows that Theorem 10.3 cannot be improved to countable models of $R C A_{0}$. A proof of the following theorem can be found in Section 10.2

Theorem 10.5. $S R T_{2}^{2}$ is not $\Pi_{4}^{0}$-conservative over $R C A_{0}$.

A proof of Lemma 10.4 can be found in Section 10.1. A proof of Theorem 10.5 can be found in Section 10.2.

10.1. The proof of Lemma 10.4. This argument will be based on the arguments presented in Sections 5.2 and 9.3. Fix a model $\mathcal{M}=(\mathbb{X}, \mathcal{S},+, \times, 0,1,<)$ of $W K L_{0}+I \Sigma_{2}$. Fix $f$ as in Lemma 10.4 . 
Let $F(x)=\lim _{s} f(x, s)$ and $A_{i}=F^{-1}(i)$ for $i \leq 1$. Except for the sets $A_{0}, A_{1}, G, Z$ and the function $F$, we will assume in this subsection that all numbers and sets mentioned are in the ground model.

Via forcing we will add an unbounded set $G$ such that either $G \subseteq A_{0}$ or $G \subseteq A_{1}$ while preserving $I \Sigma_{2}$. Without loss of generality we can assume that for all $\mathcal{M}$-infinite sets $X \in \mathcal{S}$, it is not the case that $X \subseteq A_{0}$ or $X \subseteq A_{1}$. Our first task is deciding whether $G \subseteq A_{0}$ or $G \subseteq A_{1}$.

We force over $\mathcal{M}$ using the conditions $(D, L)$ where $D$ is an $\mathcal{M}$-finite set, $L$ is an $\mathcal{M}$-infinite set in $\mathcal{S}$ and every element of $D$ is less than every element of $L$.

10.1.1. $A_{0}$ or $A_{1}$ ? We will build $G \subseteq A_{0}$ if for all $(D, L)$ and finite sets of formulas $S,(D, L)$ is $S$-large $\emptyset$ implies $\left(D, L \cap A_{0}\right)$ is $S$-large $\emptyset$. (For a definition of large $\emptyset$, see Definition 5.11.) Let $A_{i}=A_{0}, \tilde{L}=\mathbb{X}$ and $W=\emptyset$.

Otherwise, we will use $A_{1}$. Let $\tilde{D}, \tilde{L}$ and $\tilde{S}$ be the counterexample. Let $W$ be a function such that $|W|=1$ and $D_{W(0)}=\tilde{D}, L_{W(0)}=\tilde{L}$ and $S_{W(0)}=\tilde{S}$. Now it is the case that for all $(D, L)$ and finite sets $S$ of formulas, $(D, L)$ is $S$-large $W$ implies $\left(D, L \cap A_{1}\right)$ is $S$-large $_{W}$. (This follows exactly as in the proof of Lemma 5.7.) Let $A_{i}=A_{1}$.

10.1.2. The rest. We will restrict ourselves to using conditions $(D, L)$ where $D \subset A_{i}$ and $(D, L)$ is $\emptyset$-large ${ }_{W} \cdot(\emptyset, \tilde{L})$ will be our initial condition. Otherwise, the rest of the argument goes exactly like the argument in Section 9.3 using $S$-large ${ }_{W}$ conditions rather than $S$-large conditions. We leave the verification of this to the reader except for the following minor comments.

In ensuring that $G$ contains an element $\geq a$ by extending a given condition $(D, L)$, we use the fact that $L \cap A_{i}$ is unbounded, which follows from our hypothesis that $A_{1-i}$ has no unbounded subset which is a real of $\mathcal{M}$.

While the definition of smallness changed slightly from Section 9.3 , its complexity remains unchanged.

Lemma 10.6. The definition of a condition $(D, L)$ being $S$-small ${ }_{W}$ is $\Sigma_{2}^{0}$.

Proof. As noted before (see the the proof of Lemma 9.9) the definition of $S$-small $W$ boils down to the form "there exists $n^{\prime}$, a set $\left(\vec{x}_{j}: j<n^{\prime}\right)$ and finite sets $\left(D_{j}: j<n\right)$ (for all $j, D_{j} \subset A_{|W|}$ ) and something $\left.\Pi_{1}^{0, L}\right)$." Now " $D_{j} \subset A_{|W|}$ " can be replaced with "there exists $t$, for all $s \geq t$, 
for all $x, x \in D_{j}$ implies $f(x, s)=|W|$." Here we are using that $\mathcal{M}$ satisfies $B \Pi_{1}$.

10.1.3. A failed improvement; Why $I \Sigma_{2}$ is needed. One may wonder why we cannot just use the argument in Section 9.2.1 here. If this were possible, we could prove a stronger result: Let $\mathcal{M}$ be a model of $W K L_{0}$. Let $f(x, s)$ be a function in $\mathcal{M}$ such that for all $x$ and $s, f(x, s)<2$, and for all $x, \lim _{s} f(x, s)$ exists. It is possible to add an unbounded set $G$ while preserving $I \Sigma_{1}$ such that for some $j<2$, for all $x \in G$, $\lim _{s} f(x, s)=j$.

First of all this improved result would lead to the result: $R C A_{0}+R T_{2}^{2}$ is $\Pi_{1}^{1}$-conservative over $R C A_{0}+I \Sigma_{1}$. But this contradicts Theorem 10.5 . Let's take a closer look to see where the argument breaks down.

Let $\psi(G)$ be a $\Sigma_{1}^{0}$ sentence. Here whether we can extend a given condition $(D, L)$ to a condition $\left(D^{*}, L^{*}\right)$ such that $\left(D^{*}, L^{*}\right)$ forces $\psi(G)$ is a $\Sigma_{1}^{0}$-question. $D^{*}$ must be a subset of $(D \cup L) \cap A_{i}$ such that $\psi\left(D^{*}\right)$. But $A_{i}$ is not a set in the ground model. Asking if $x \in A_{i}$ is $\Delta_{2}^{0}$. Hence in this case whether we can extend a given condition $(D, L)$ to a condition $\left(D^{*}, L^{*}\right)$ such that $\left(D^{*}, L^{*}\right)$ forces $\psi(G)$ is $\Sigma_{2}^{0}$. Hence we cannot just use $I \Sigma_{1}$ as we did in Section 9.2.1, we need $I \Sigma_{2}$.

10.2. The proof of Theorem 10.5. Slaman's proof of Theorem 2.9 involves two lemmas; Seetapun and Slaman [1995, Lemmas 3.4 and 3.5]. The first is well-known; the second is not. By examining how these two lemmas are used (see the proof of Seetapun and Slaman [1995, Theorem 3.6]), we can see that it is enough to alter Seetapun and Slaman [1995, Lemma 3.5] so that the function $F$ (the partition or 2-coloring of all pairs) produced is stable. For this task we will adopt the notation of Seetapun and Slaman [1995, Lemmas 3.4 and 3.5]. Since we do not have to make major changes we will just present the needed changes to Seetapun and Slaman [1995, Lemma 3.5].

First we will require that $h_{0}[s+1], \ldots, h_{a}[s+1]$ be sets of cardinality $2 a$ rather cardinality $a$. We will have to choose $x_{i}$ carefully. Define $x_{i}[s]$ by recursion for $i<a$; Let $x_{i}[s]$ be such that $x_{i}[s] \in h_{i}[s]$, for all $j<i$, $x_{i}[s] \neq x_{j}[s]$ and for all $j \leq i, x_{i}[s] \neq a_{j}[s]$. Since for each standard $n$ $\lim _{s} a_{n}[s]=a_{n}$ exists and we have changed the size of $h_{i}[s]$ to $2 a$, if $i$ is standard then $\lim _{s} x_{i}[s]$ exists. Define $F\left(x_{i}[s+1], s+1\right) \neq F\left(l_{1}, l_{2}\right)$, where $l_{1}$ and $l_{2}$ are the first two elements (under $<$ ) of $h_{a}[s+1]$; otherwise for $x \leq s+1, F(x, s+1)=0$. Pick some $x$. Since the sequence of $a_{n}$ 's (for standard $n$ ) lists every number in our model, either $x=\lim x_{i}[s]$ or for only finitely many $s$ there is an $i$ such that $x=x_{i}[s]$. In either case, $\lim _{s} F(x, s)$ exists. 


\section{Finitely MANY COLORS}

The following is the main result of this section.

Theorem 11.1. $R T_{<\infty}^{2}+R C A_{0}+I \Sigma_{3}$ is conservative for arithmetic statements over $R C A_{0}+I \Sigma_{3}$.

This is a consequence of the following theorem.

Theorem 11.2. Every countable topped model of $R C A_{0}+I \Sigma_{3}$ is an $\omega$-submodel of some countable model of

$W K L_{0}+I \Sigma_{3}+R T_{<\infty}^{2}$.

In fact Theorem 11.2 implies that $R T_{<\infty}^{2}+W K L_{0}+I \Sigma_{3}$ is conservative for arithmetic statements over $R C A_{0}+I \Sigma_{3}$. Theorem 11.2 is deduced from the following result.

Lemma 11.3. Assume $\mathcal{M}$ is a countable model of $R C A_{0}+I \Sigma_{3}$. Let $f(x, s)$ be a function coded in $\mathcal{M}$ and $k$ be a number in $\mathcal{M}$ such that for all $x$ and $s, f(x, s)<k$, and for all $x, \lim _{s} f(x, s)$ exists. Furthermore assume $\mathcal{M}$ has a set $T$ which uniformly codes a Scott set $\mathcal{S}_{T}$ containing $f$. It is possible to add a unbounded set $G$ to $\mathcal{M}$ while preserving $I \Sigma_{3}$ such that for some $j<k$, for all $x \in G, \lim _{s} f(x, s)=j$.

Proof of Theorem 11.2. Let $\mathcal{M}=(\mathbb{X}, \mathcal{F},+, \times, 0,1,<)$ be a countable model of $R C A_{0}+I \Sigma_{3}$, and let $\mathcal{M}$ be topped by $D$.

Choose some $f$ and $k$ as in Lemma 11.3. Apply Lemma 8.20 to get $\mathcal{M}^{\prime}$ such that $\mathcal{M}^{\prime}$ is a model of $R C A_{0}+I \Sigma_{3}, \mathcal{M}^{\prime}$ has a set $T$ which uniformly codes a Scott set $\mathcal{S}_{T}$ containing $f$, and $T \oplus D$ witnesses that $\mathcal{M}^{\prime}$ is topped. Apply Lemma 11.3 to add an unbounded set $G$ to $\mathcal{M}^{\prime}$ while preserving $I \Sigma_{3}$ such that for some $j<k$, for all $x \in G, \lim _{s} f(x, s)=j$. Then apply Lemma 6.7 to get a model $\mathcal{M}^{\prime \prime}$ of $R C A_{0}+I \Sigma_{3} . \mathcal{M}^{\prime \prime}$ is topped by $G \oplus T \oplus D$.

Choose some $\left\{R_{i}\right\}_{i \in \omega}$ a sequence of sets which is coded by $C$ in $\mathcal{M}^{\prime \prime}$. Apply Lemma 8.20 to get $\mathcal{M}^{\prime \prime \prime}$ such that $\mathcal{M}^{\prime \prime \prime}$ is a model of $R C A_{0}+I \Sigma_{3}$, $\mathcal{M}^{\prime \prime \prime}$ has a set $T^{*}$ which uniformly codes a Scott set $\mathcal{S}_{T^{*}}$ containing $C$, and $\mathcal{M}^{\prime \prime \prime}$ is topped by $T^{*} \oplus G \oplus T \oplus D$. Apply Lemma 9.7 to add a $\vec{R}$ cohesive set $G^{*}$ to $\mathcal{M}^{\prime \prime \prime}$ while preserving $I \Sigma_{3}$. Then apply Lemma 6.7 to get a model $\mathcal{M}^{*}$ of $R C A_{0}+I \Sigma_{3}$. $\mathcal{M}^{*}$ is topped by $G^{*} \oplus T^{*} \oplus G \oplus T \oplus D$.

Iterate the process infinitely many times, ensuring that for every $f$ and $k$, as in Lemma 11.3, an unbounded set $G$ is added such that for some $j<k$, for all $x \in G, \lim _{s} f(x, s)=j$ and for every such coded sequence of sets $\left\{R_{i}\right\}$ a $\vec{R}$-cohesive set $G$ is added. The resulting model is a model of $W K L_{0}+I \Sigma_{3}+C O H+D_{<\infty}^{2}$. By Lemma 7.12, $R C A_{0}+D_{<\infty}^{2}$ implies $S R T_{<\infty}^{2}$ and, by Lemma 7.13, $R C A_{0}+C O H+S R T_{<\infty}^{2}$ implies $R T_{<\infty}^{2}$. 
A proof of Lemma 11.3 will appear in Section 11.1. However, the following result shows that these theorems cannot be improved to $I \Sigma_{2}$.

Theorem 11.4. $R C A_{0}+S R T_{<\infty}^{2} \vdash B \Sigma_{3}$.

Corollary 11.5. $R C A_{0}+R T_{<\infty}^{2} \vdash B \Sigma_{3}$.

This improves work of Mytilinaios and Slaman [1994] who showed $R C A_{0}+R T_{<\infty}^{2} \vdash I \Sigma_{2}$. Since $B \Sigma_{3}$ is stronger than $I \Sigma_{2}$ (see Kaye [1991] or Hájek and Pudlák [1993]), we have that $R T_{2}^{2}$ does not imply $R T_{<\infty}^{2}$ (and $S R T_{2}^{2}$ does not imply $S R T_{<\infty}^{2}$ ). A proof of Theorem 11.4 can be found in Section 11.2

11.1. A Proof of Lemma 11.3. The argument is similar to that in Section 9.4.1, although the forcing conditions are modified as in Section 10.1. Fix a model $\mathcal{M}, f, k, T$ and $S_{T}$ as in Lemma 11.3. Let $F(x)=\lim _{s} f(x, s)$ and $A_{i}=F^{-1}(i)$. It is enough to add an unbounded set $G$ to $\mathcal{M}$ such that for some $i, G \subseteq A_{i}$ while preserving $I \Sigma_{3}$.

Except for the sets $A_{i}, G$ and the function $F$, we will assume in this subsection that all numbers and sets mentioned are in the ground model. Clearly, $A_{i}$ is $\Delta_{2}^{0, f}$ and hence $\Delta_{2}^{0, T}$. Lemma 10.6 applies; so the while the definition of smallness has slightly changed (from the notion used in Section 9.4) its complexity remains unchanged.

We force over $\mathcal{M}$ using the conditions $(D, L)$ where $D$ is $\mathcal{M}$-finite, $L \in S_{T}$ is an $\mathcal{M}$-infinite set and every element of $D$ is less than every element of $L$.

Our first task is to decide for which $i<k, G \subseteq A_{i}$.

11.1.1. Which $A_{i}$ ? We would like to find an $i$ and a function $W$ such that $|W|=i$ and for all conditions $(D, L)$ and finite sets of $\Sigma_{2}^{0}$ formulas,

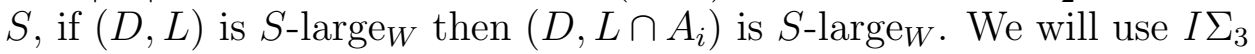
to find such an $i$ and $W$.

Lemma 11.6. "There is a $W$ such that for all $j \leq i,\left(D_{W(j)}, L_{W(j)}\right)$ is $S_{W(j)}$-large $_{W\lceil j}$ and $\left(D_{W(j)}, L_{W(j)} \cap A_{j}\right)$ is $S_{W(j)}-$ small $_{W \uparrow j}$ " is a $\Sigma_{3}^{0, T}$ formula ( $i$ is a free variable).

Proof. There is a $\Pi_{2}^{0}$ formula $\lambda(S, D, L)$ such that whenever $S$ is a (code for) an $M$-finite set of $\Sigma_{2}^{0}$ formulas, $D$ is an $\mathcal{M}$-finite set, and $L \in \mathcal{S}_{T}$, then $\lambda(S, D, L)$ holds in $\mathcal{M}$ iff $(D, L)$ is an $S$-large condition (this uses a number of previous lemmas including Lemma 10.6). Since $A_{i}$ is $\Delta_{2}^{0, T}$, whether $\left(D, L \cap A_{i}\right)$ is an $S$-small condition is $\Sigma_{3}^{0, T}$. The quantifier over $W$ can be replaced by a number quantifier, using a parameter for $T$. The rest is routine quantifier counting. 
Let $\xi(i)$ be the $\Sigma_{3}^{0, T}$ formula described by the above lemma. Consider the set $B=\{b: \neg \xi(b)\} . B$ is a $\Pi_{3}^{\mathbf{0}}$ set. Hence by $I \Sigma_{3}$ in the ground model if nonempty $B$ must have a least element. The next lemma shows that $B$ is nonempty and the least element must be less than $k$. (The lemma and its proof are based on Lemma 5.12.)

Lemma 11.7. Let $l=k-1$. Then $\neg \xi(l)$.

Proof. We argue informally. Assume $\xi(l)$. Let $W$ witness the satisfaction of $\xi(l)$. Thus $|W| \geq k$. Let $D=\tilde{D}_{W(l)}, L=\tilde{L}_{W(l)}, S=\tilde{S}_{W(l)}$.

For $n \leq l$, we have that $\left(\tilde{D}_{W(n)}, \tilde{L}_{W(n)} \cap A_{n}\right)$ is $\tilde{S}_{W(n)}$-small ${ }_{W \uparrow(n)}$. Let $n<l$ and inductively assume that $\left(D, L \cap\left(\sqcup_{i<n} A_{i}\right)\right)$ is $\emptyset$-small ${ }_{W\lceil n}$. Since $(D, L)$ is $W$-acceptable, $L \subseteq \tilde{L}_{W(n)}$ and hence $\left(\tilde{D}_{W(n)}, L \cap A_{n}\right)$ is $\tilde{S}_{W(n)}-\operatorname{small}_{W \uparrow(n)}$. So $\left(\tilde{D}_{W(n)}, L \cap\left(\sqcup_{i<n+1} A_{i}\right)\right)$ is $\tilde{S}_{W(n)}-\operatorname{small}_{W \uparrow(n)}$. Hence $\left(D, L \cap\left(\sqcup_{i<n+1} A_{i}\right)\right)$ is $\emptyset$-small $W_{W \uparrow(n+1)}$. Therefore $\left(D, L \cap\left(\sqcup_{i<l} A_{i}\right)\right)$ is $\emptyset$-small ${ }_{W\lceil l}$.

Therefore if $\left(D, L \cap A_{l}\right)$ is $S$-small s $_{W l}$ then $(D, L)$ must be $S$-small s $_{\lceil l}$. This contradicts the choice of $D, L$ and $S$.

To make this formal we need the fact that $\mathcal{M}$ is a model of $I \Sigma_{3}$ to prove a lemma similar to Lemma 9.9 and to make the above induction showing $\left(D, L \cap\left(\sqcup_{i<l} A_{i}\right)\right)$ is $\emptyset$-small - $_{\lceil l}$ hold in $\mathcal{M}$.

11.1.2. The rest. Let $i$ be the least element of the set $B$. Then $i<k$. Let $W$ witness the satisfaction of $\xi(i-1)$ (if $i=0$ let $W=\emptyset$ ). Hence $|W|=i-1$ and for all conditions $(D, L)$ and all $\mathcal{M}$-finite sets $S$ of $\Sigma_{2}^{0}$ formulas, if $(D, L)$ is $S$-large $W$, then $\left(D, L \cap A_{i}\right)$ is $S$-large $_{W}$. We will restrict ourselves to using conditions $(D, L)$ where $D \subset A_{i}$ and $(D, L)$ is $\emptyset$-large ${ }_{W}$. $\left(\emptyset, \tilde{L}_{W(i-1)}\right)$ will be our initial condition (if $i=0$ use $(\emptyset, \mathbb{X})$ as the initial condition).

Otherwise, the rest of the argument goes exactly like the argument in Section 9.4 using $S$-large $W$ conditions rather than $S$-large conditions. We leave this to the reader to verify except for one minor comment. If $(D, L)$ is any condition such that where $D \subset A_{i}$ and $(D, L)$ is $\emptyset$-large ${ }_{W}$, then $L \cap A_{i}$ is unbounded (since $\left(D, L \cap A_{i}\right)$ is $\emptyset$-large $W$ ). Thus, it will be possible to ensure that $G$ is unbounded.

11.2. A Proof of Theorem 11.4. By Mytilinaios and Slaman [1994, Proposition 5.2], $R C A_{0}+R T_{<\infty}^{2}$ implies $B \Sigma_{2}$. However their proof shows something stronger: $R C A_{0}+S R T_{<\infty}^{2}$ implies $B \Sigma_{2}$. We will use this below.

We work in a nonstandard model $\mathcal{M}$ and suppose that we are given a failure of $B \Sigma_{3}$. That is, we are given a number $a$ and a $\Sigma_{3}$ formula 
$(\exists x)(\forall y)(\exists z) \varphi(w, x, y, z)$, with free variable $w$, such that the following conditions hold.

(1) For all $w$ less than $a,(\exists x)(\forall y)(\exists z) \varphi(w, x, y, z)$.

(2) For all $s$, there is a $w$ less than $a$ such that $\neg(\exists x<s)(\forall y)(\exists z) \varphi(w, x, y, z)$.

Let $\mathbb{X}$ be the set of numbers of $\mathcal{M}$. We define a coloring $\mathcal{C}$ of $[\mathbb{X}]^{2}$ into $a$-many colors which is $\Delta_{1}^{0}$ over $\mathcal{M}$, ensure that $\mathcal{C}$ is stable, and ensure that for each $w$ less than $a$ the set of $s$ such that $\mathcal{C}$ is stable with value $w$ for pairs which begin with $s$ is bounded in $\mathbb{X}$. Basically, we want $\lim _{t \rightarrow \infty} \mathcal{C}(s, t)$ to equal some $w$ less than $a$ such that $\neg(\exists x<s)(\forall y)(\exists z) \varphi(w, x, y, z)$ (we use $\mathcal{C}(x, y)$ as shorthand for $\mathcal{C}(\{x, y\}))$. There is such a $w$ by Item 2 above. During stage $t$, we define $\mathcal{C}(s, t)$ for all $s$ less than $t$ and let $\mathcal{C}(s, t)$ be our best guess for such a $w$.

Say that a number $w<a$ is released for the $y^{*}$ th time during stage $t$ relative to $s$ if $(\exists x<s)\left(\forall y<y^{*}\right)(\exists z<t) \varphi(w, x, y, z)$. We define $\mathcal{C}(s, t)$ to be the least $w$ less than $a$ such that the number of times that $w$ has been released relative to $s$ during stage $t$ is minimized in comparison to other numbers less than $a$.

First, check that $\mathcal{C}$ is stable. By Item 2, there is a $w$ less than $a$ such that $\neg(\exists x<s)(\forall y)(\exists z) \varphi(w, x, y, z)$. Fixing such a $w$ and looking at the numbers $x$ less than $s$, we have a function mapping $x$ to the least $y$ such that $\neg(\exists z) \varphi(w, x, y, z)$. By $B \Sigma_{2}$, there is a bound $y^{*}$ on the range of this function and a bound on all of the $z$ 's associated with $y$ 's smaller than $y^{*}$. So there is a $w$ which is released no more than $y^{*}$ times relative to $s$ during all sufficiently large stages $t$. The stability of $\mathcal{C}$ follows by arguing that the minimum number of releases reaches a limit and then that the minimum $w$ for this number of releases also reaches a limit.

Now, check that for each $w$ less than $a, w$ can be the stable value for only boundedly many $s$. Fix $w$. By Item $1,(\exists x)(\forall y)(\exists z) \varphi(w, x, y, z)$. Fix $x$ so that $x$ is a witness to the leading existential quantifier of this formula. If $s$ is greater than $x$ then $w$ will be released $y^{*}$ times during each stage $t$ such that $\left(\forall y<y^{*}\right)(\exists z<t) \varphi(w, x, y, z)$. Thus, the number of times that $w$ is released relative to $s$ during stage $t$ goes to infinity as $t$ increases. Consequently $w$ cannot be the eventual value of $\mathcal{C}(s, t)$ as $t$ increases.

\section{More Computability Results}

12.1. Extension to $n$-tuples. The following result extends the existence of $\mathrm{low}_{2}$ infinite homogeneous sets from colorings of pairs to colorings of $n$-tuples. 
Theorem 12.1. For each $n \geq 2$ and each computable 2-coloring of $[\mathbb{N}]^{n}$, there is an infinite homogeneous set $A$ with $A^{\prime \prime} \leq_{T} 0^{(n)}$.

Proof. This is proved in relativized form by induction on $n$. The base case $n=2$ is proved by relativizing Theorem 3.1. Now assume the result for $n$ in order to prove it for $n+1$. For notational simplicity, we prove it in unrelativized form. Let $\mathcal{C}$ be a computable 2 -coloring of $[\mathbb{N}]^{2}$. By Jockusch $[1972$, Lemma 5.4] there is a pre-homogeneous set $A$ with $A^{\prime} \leq_{T} 0^{\prime \prime}$. (A set $A$ is pre-homogeneous if any two $(n+1)-$ element subsets of $A$ with the same first $n$ elements are assigned the same color by $\mathcal{C}$.) Now $\mathcal{C}$ induces a coloring $\mathcal{C}^{\prime}$ on $[A]^{n}$, i.e., for $D \in[A]^{n}$, $\mathcal{C}^{\prime}(D)=\mathcal{C}(D \cup\{a\})$, where $a \in A$ and $a>\max (D)$ (this is well-defined since $A$ is pre-homogeneous). Applying the inductive hypothesis to the $A$-computable coloring $\mathcal{C}^{\prime}$, one obtains a homogeneous set $X$ for $\mathcal{C}^{\prime}$ such that $X^{\prime \prime} \leq_{T} A^{(n)} \leq_{T} 0^{(n+1)}$. Since every homogeneous set for $\mathcal{C}^{\prime}$ is homogeneous for $\mathcal{C}$, the induction is complete.

The above is best possible since, by Theorem $2.5 \mathrm{iv}$, there is a computable 2-coloring of $[\mathbb{N}]^{n}$ with $0^{(n-2)} \leq_{T} A$ (and so $0^{(n)} \leq_{T} A^{\prime \prime}$ ) for each infinite homogeneous set $A$.

12.2. Avoiding cones. The next result extends Seetapun's cone avoidance theorem (Theorem 2.7) to colorings of $n$-tuples and also, for $n=2$, gives a homogeneous set which is not high.

Theorem 12.2. For each $n \geq 2$, each computable $k$-coloring $\mathcal{C}$ of $[\mathbb{N}]^{n}$, and any sequence of sets $C_{0}, C_{1}, \ldots$ with $(\forall i)\left[C_{i} \mathbb{Z}_{T} 0^{(n-2)}\right]$, there is an infinite homogeneous set $A$ with $A^{\prime} \Varangle_{T} 0^{(n)}$ and $(\forall i)\left[C_{i} \not_{T} A\right]$.

Proof. This is proved in relativized form by induction on $n$. First consider the base step $n=2$, which we prove in unrelativized form with $k=2$ for notational convenience. Let a computable 2-coloring $\mathcal{C}$ of $[\mathbb{N}]^{n}$, and a sequence of noncomputable sets $C_{0}, C_{1}, \ldots$ be given. We must construct an infinite homogeneous set $A$ with $A^{\prime} \Varangle_{T} 0^{\prime \prime}$ and $(\forall i)\left[C_{i} \not_{T} A\right]$. This will be done by applying Theorem 3.6 and the argument which was used to prove Jockusch and Stephan 1993 , Theorem 4.6], which is the analogous cone avoidance result for non-high cohesive degrees. (In Jockusch and Stephan [1993, Theorem 4.6] a should be replaced by $\mathbf{b}$ in the statement of the result for consistency with the notation used in the proof of that result.)

Let $\mathbf{c}_{\mathbf{i}}$ be the degree of $C_{i}$. Define inductively a sequence of degrees $\mathbf{d}_{0}, \mathbf{d}_{1}, \ldots\left(\mathbf{d}_{i} \nsucceq \mathbf{0}^{\prime \prime}\right)$ as follows. Let $\mathbf{d}_{0}=\mathbf{0}^{\prime}$. If $\mathbf{d}_{i} \cup \mathbf{c}_{\mathbf{i}} \geq \mathbf{0}^{\prime \prime}$, then let $\mathbf{e}_{i}=\mathbf{c}_{i}$ else $\mathbf{e}_{i}=\mathbf{0}^{\prime}$; in both cases let $\mathbf{d}_{i+1}=\mathbf{d}_{i} \cup \mathbf{e}_{\mathbf{i}}$. By Spector's Theorem [Spector, 1956] (see Odifreddi [1989, p. 485]) the ideal generated 
by the degrees $\mathbf{d}_{i}$ has an exact pair $\mathbf{f}$ and $\mathbf{g}$ such that the degrees $\mathbf{e}_{i}$ are uniformly recursive in both $\mathbf{f}$ and $\mathbf{g}$. Since $\mathbf{0}^{\prime \prime}$ does not belong to the ideal, one half of the exact pair is not above $\mathbf{0}^{\prime \prime}$, say $\mathbf{g} \geq \mathbf{0}^{\prime \prime}$. Posner and Robinson [1981, Theorem 3] showed that there is a degree a such that $\mathbf{a}^{\prime}=\mathbf{g}$ and $\mathbf{a}^{\prime}=\mathbf{a} \cup \mathbf{e}_{\mathbf{i}}$ for all $i$. By Jockusch and Soare [1972, Theorem $2.4]$ relativized to $\mathbf{a}^{\prime}$ there is a degree $\mathbf{d}>>\mathbf{a}^{\prime}$ which is hyperimmunefree relative to $\mathbf{a}^{\prime}$. Hence by Theorem 3.6 relativized to a there is a degree $\mathbf{b}$ which contains an infinite set $B$ which is homogeneous for the given coloring $\mathcal{C}$ and such that $(\mathbf{b} \cup \mathbf{a})^{\prime} \leq \mathbf{d}$.

Since $\mathbf{a}^{\prime} \cup \mathbf{0}^{\prime \prime}$ is computably enumerable but not computable in $\mathbf{a}^{\prime}$, $\mathbf{a}^{\prime} \cup \mathbf{0}^{\prime \prime}$ is hyperimmune relative to $\mathbf{a}^{\prime}$, and hence $\mathbf{a}^{\prime} \cup \mathbf{0}^{\prime \prime}$ is also hyperimmune relative to $\mathbf{b}^{\prime}$. Thus $\mathbf{0}^{\prime \prime} \not \leq \mathbf{b}^{\prime}$ so $\mathbf{b}$ is not high.

Assume now for a contradiction that $\mathbf{c}_{i} \leq \mathbf{b}$. If $\mathbf{e}_{i}=\mathbf{c}_{i}$, then $\mathbf{b}=\mathbf{b} \cup \mathbf{e}_{\mathbf{i}} \geq \mathbf{a} \cup \mathbf{e}_{\mathbf{i}}=\mathbf{a}^{\prime}$ and $\mathbf{b}^{\prime} \geq \mathbf{a}^{\prime \prime} \geq \mathbf{0}^{\prime \prime}$. Otherwise, $\mathbf{c}_{i} \cup \mathbf{d}_{\mathbf{i}} \geq \mathbf{0}^{\prime \prime}$. Then $\mathbf{b}^{\prime} \geq \mathbf{c}_{i} \cup \mathbf{a}^{\prime}=\mathbf{c}_{\mathbf{i}} \cup \mathbf{g} \geq \mathbf{c}_{\mathbf{i}} \cup \mathbf{d}_{\mathbf{i}} \geq \mathbf{0}^{\prime \prime}$. Both cases contradict the above assertion that $\mathbf{b}$ is not high, so $\mathbf{c}_{i} \not \leq \mathbf{b}$. This completes the proof of the theorem for the case $n=2$.

For the induction step, assume the theorem holds for $n$ (in relativized form). We prove it for $n+1$, but for notational convenience assume that $k=2$ and prove it in unrelativized form. Let a computable 2 -coloring $\mathcal{C}$ of $[\mathbb{N}]^{n+1}$, and a sequence of sets $C_{0}, C_{1}, \ldots$ with $(\forall i)\left[C_{i} \mathbb{Z}_{T} 0^{(n-1)}\right]$ be given. We must construct an infinite homogeneous set $B$ with $B^{\prime} \Varangle_{T} 0^{(n+1)}$ and $(\forall i)\left[C_{i} \not \mathbb{L}_{T} B\right]$. By Jockusch [1972, Lemma 5.4] there is a pre-homogeneous set $A$ with $A^{\prime} \leq_{T} 0^{\prime \prime}$. Then $\mathcal{C}$ induces an $A$-computable 2 -coloring $\widehat{\mathcal{C}}$ of $[A]^{n}$ (for more details see the proof of Theorem 12.1). Note that for each $i, C_{i} \mathbb{Z}_{T} A^{(n-2)}$, since otherwise we obtain $C_{i} \leq_{T} A^{(n-2)} \leq_{T} 0^{(n-1)}$. Since we are assuming that the theorem holds for $n$ relative to $A$, there is an infinite set $B$ which is homogeneous for $\widehat{\mathcal{C}}$, and hence for $C$, such that $B^{\prime} \Varangle_{T} A^{(n)}$ and $(\forall i)\left[C_{i} \mathbb{Z}_{T} B \oplus A\right]$. It then follows that $B^{\prime} \Varangle_{T} 0^{(n+1)}$ and $(\forall i)\left[C_{i} \mathbb{Z}_{T} B\right]$, which completes the induction.

12.3. Uniformity and Theorem 3.6. The only nonuniform step in the proof of Theorem 3.1 is the use of Theorem 3.6 (or Theorem 3.7). The following is a uniform version of Theorem 3.6 which is useful in Hummel and Jockusch [n.d.].

Theorem 12.3. There is a function $f \leq_{T} 0^{(3)}$ such that whenever the number $a$ is a $\Delta_{2}^{0}$ index of a $\Delta_{2}^{0}$ set $A, H^{\prime \prime}=\{f(a)\}^{0^{\prime \prime}}$ for some infinite set $H$ contained in or disjoint from $A$.

Proof. The proof is based on a slight modification of the proof of Theorem 3.6 found in Section 4.2. Here we will require that the sets $L$ in 
the forcing conditions $(D, L)$ lie in a fixed, uniformly low Scott set $\mathcal{S}$. (As we explained in Section 8.4, such a set exists). As all sets in $\mathcal{S}$ are low, the proof of Theorem 3.6 goes through with this restricted set of forcing conditions. It is a $\Pi_{3}^{0}$ predicate of $a$ to say that $a$ is a $\Delta_{2}^{0}$ index, and we can define $f(a)$ arbitrarily if it is not. Assume now that it is, and let $A$ be the set of which $a$ is a $\Delta_{2}^{0}$ index. Fix a set $B$ of degree $\mathbf{d}$ as in the proof of Theorem 3.6 in Section 4.2. The construction produces effectively from $a$ two numbers $b$ and $c$ such that at least one of the following conditions holds:

(A) Some set $S \in \mathcal{S}$ is an infinite subset of $A$ or $\bar{A}$,

(B) $\{b\}^{B}=X^{\prime}$ for some infinite $X \subseteq A$, or

(C) $\{c\}^{B}=Y^{\prime}$ for some infinite $Y \subseteq \bar{A}$.

(For example, $\{b\}^{B}(e)=1$ if there exists $i$ such that $e \in X^{\prime}$ is forced at stage $2\langle e, i\rangle$.) Furthermore, if $\{b\}^{B}$ is total and Condition (A) is false, then (B) holds. An analogous statement holds for $\{c\}^{B}$ and Condition $(C)$.

Since $\mathcal{S}$ is uniformly computable from $T$ where $T$ is low, the Condition (A) is a $\Sigma_{3}^{0}$ predicate of $a$. If Condition (A) holds, one may $0^{(3)}$-effectively find a lowness index for an infinite subset of $A$ or $\bar{A}$ which is in $\mathcal{S}$, and from that a low 2 index of the set, i.e. an appropriate value of $f(a)$. Suppose now that Condition $(\mathrm{A})$ is false. The predicate " $\{d\}^{B}$ is total" is a $\Pi_{3}^{0}$ predicate of $d$ and $a$ and either (B) or (C) holds, so one may $0^{(3)}$-effectively choose one of the two Conditions (B), (C) which holds. It is then easy to compute $f(a)$ as required, using that $\mathbf{d}$ is low over $\mathbf{0}^{\prime}$.

(It is possible to make modifitions to the proof found in Section 5.2 to prove the above result.)

12.4. Jump universal. The following results show a close degreetheoretic connection between degrees $\mathbf{d}>>\mathbf{0}^{\prime}, \mathrm{r}-$ cohesive sets and infinite sets homogeneous for computable colorings of pairs.

Theorem 12.4 (Jockusch and Stephan [1993]). The following are equivalent for any degree $\mathbf{d}$ :

(i) There is an $r$-cohesive (p-cohesive) set with jump of degree $\mathbf{d}$.

(ii) $\mathbf{d}>>\mathbf{0}^{\prime}$

Proof. The implication (ii) $\rightarrow$ (i) is Corollary 4.5. For the implication $(\mathrm{i}) \rightarrow$ (ii), suppose that $A$ is a p-cohesive set. Let $g$ be a primitive recursive $\{0,1\}$-valued function such that $\lim _{s} g(e, i, s)=\{e\}^{K}(i)$ whenever $\{e\}^{K}(i) \downarrow \leq 1$. Such a function $g$ exists by the proof of the limit lemma. Since $A$ is $\mathrm{p}-$ cohesive, $\lim _{s \in A} g(e, i, s)$ exists for all $e$ and 
$i$. Let $f(e, i)=\lim _{s \in A} g(e, i, s)$, so that $f(e, i)=\{e\}^{K}(i)$ whenever $\{e\}^{K}(i) \downarrow \leq 1$. Clearly, $f \leq_{T} A^{\prime}$, and each $K$-computable $\{0,1\}$ valued partial function has a total $f$-computable extension. Hence $\mathbf{d}=\operatorname{deg}\left(G^{\prime}\right)>>\mathbf{0}^{\prime}$.

Theorem 12.5. There is a computable 2 -coloring $\mathcal{C}$ of $[\mathbb{N}]^{2}$ such that every infinite homogeneous set $A$ has jump of degree $>>\mathbf{0}^{\prime}$.

Proof. By Theorem 12.4, it will suffice to produce a computable 2coloring $\mathcal{C}$ such that every infinite homogeneous set is $p$-cohesive.

Let $A_{0}, A_{1}, \ldots$ be an uniformly computable listing of the primitive recursive sets. If $a \neq b$ let $d(a, b)$ be the least $i$ with $A_{i}(a) \neq A_{i}(b)$. If $a<b$ and $a \in A_{i(a, b)}$ then $\mathcal{C}$ colors $\{a, b\}$ red and otherwise it colors the pair blue.

Suppose that $A$ is a homogeneous set for $\mathcal{C}$ and (for a contradiction) that $A$ is split by $A_{k}$. Choose $k$ as small as possible. Then for sufficiently large distinct numbers $a, b \in A, d(a, b) \geq k$. Then take "sufficiently large" $a, b, c \in A$ with $a<b<c, A_{k}(a) \neq A_{k}(b)$ and $A_{k}(b) \neq A_{k}(c)$, so that $d(a, b)=d(b, c)=k$. But then $\mathcal{C}$ colors $\{a, b\}$ red iff $\mathcal{C}$ colors $\{b, c\}$ blue, contradicting the homogeneity of $A$.

The proof of Theorem 3.6 in Section 4.2 shows that for every degree $\mathbf{d}>>\mathbf{0}^{\prime}$ and for every computable 2-coloring $\mathcal{C}^{\prime}$, there exists a homogeneous set $B$ (for $\mathcal{C}^{\prime}$ ) with $B^{\prime} \leq_{T} \mathbf{d}$. Hence the above coloring $\mathcal{C}$ is "jump universal" in the sense that for any homogeneous set $A$ (of $\mathcal{C}$ ) and any computable 2-coloring $\mathcal{C}^{\prime}$, there exists a homogeneous set $B$ (for $\mathcal{C}^{\prime}$ ) with $B^{\prime} \leq_{T} A^{\prime}$. Also, note that for any computable coloring $\mathcal{C}^{\prime}$ the degrees of the infinite homogeneous sets are closed upwards by Jockusch [1973, Corollary 1] and hence the degrees of the jumps of such sets are closed upwards by the relativized Friedberg completeness criterion. Hence we have the following corollary:

Corollary 12.6. The following are equivalent for any degree $\mathbf{d}$ :

(i) Every computable 2-coloring of $[\mathbb{N}]^{2}$ has an infinite homogeneous set with jump of degree $\mathbf{d}$.

(ii) $\mathbf{d}>>\mathbf{0}^{\prime}$

It is open whether there is a computable coloring $\mathcal{C}$ which is "universal" (i.e., for any infinite homogeneous set $A$ (for $\mathcal{C}$ ) and any computable 2-coloring $\mathcal{C}^{\prime}$, there exists an infinite homogeneous set $B$ (for $\left.\mathcal{C}^{\prime}\right)$ with $B \leq_{T} A$.)

\section{Conclusions And Questions}


13.1. The relationship between $2^{\text {nd }}$ order theories and statements. Figure 1 summarizes the relationship between various second order theories and statements. (The arrows are implications. The solid arrows cannot not be reversed (unless of course they have arrows in both directions). It is not known if the dashed ones can. The lack of arrows means the relations between the theories is also unknown.) The two relations that are missing from Figure 1 are the one we were able to exploit for some of our results: over $R C A_{0}, R T_{2}^{2}$ is equivalent to $C O H+S R T_{2}^{2}$ and $R T_{<\infty}^{2}$ is equivalent to $C O H+S R T_{<\infty}^{2}$.

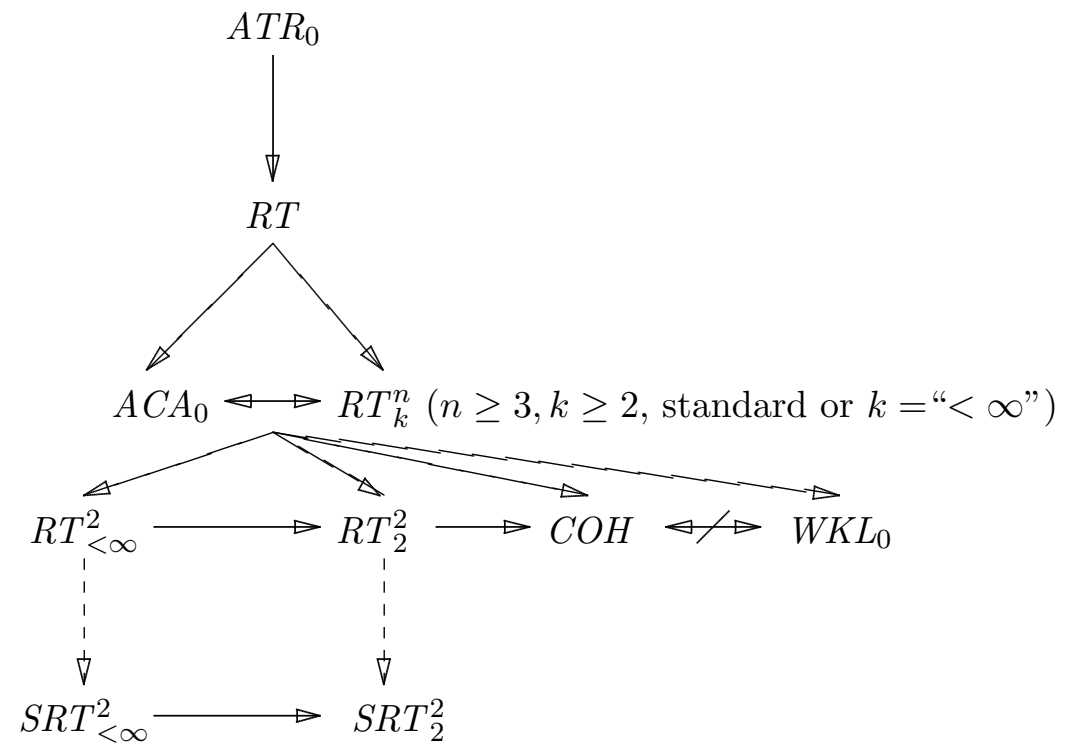

FiguRE 1. The relationship between $2^{\text {nd }}$ order theories and statements.

\subsection{First order consequences.}

Theorem 13.1. Let $(\varphi)^{1}$ be the set of first order consequences of $\varphi+R C A_{0}$.

(i) $\left(R C A_{0}\right)^{1}=\left(W K L_{0}\right)^{1}=(C O H)^{1}$.

(ii) $\left(R C A_{0}\right)^{1} \subsetneq\left(S R T_{2}^{2}\right)^{1} \subseteq\left(R T_{2}^{2}\right)^{1} \subseteq\left(I \Sigma_{2}\right)^{1}$.

(iii) $\left(I \Sigma_{2}\right)^{1} \subsetneq\left(B \Sigma_{3}\right)^{1} \subseteq\left(S R T_{<\infty}^{2}\right)^{1} \subseteq\left(R T_{<\infty}^{2}\right)^{1} \subseteq\left(I \Sigma_{3}\right)^{1}$.

(iv) $\left(I \Sigma_{3}\right)^{1} \subsetneq P A=\left(R T_{2}^{3}\right)^{1}=\left(R T_{n}^{k}\right)^{1}$ (for any fixed $k \geq 3$ and $n \geq 2$ ).

13.3. Reverse Mathematics Questions. Perhaps the most interesting question in this vein is: 
Question 13.2. Is $R C A_{0}+R T_{2}^{2} \Pi_{2}^{0}$-conservative over $R C A_{0}$ ? In particular, does $R C A_{0}+R T_{2}^{2}$ prove the consistency of $P^{-}+I \Sigma_{1}$ ? Does $R C A_{0}+R T_{2}^{2}$ prove that the Ackermann function is total?

It is known that the provably total recursive functions in $R C A_{0}$ are exactly the primitive recursive functions. This characterizes the $\Pi_{2}^{0}$ sentences provable from $R C A_{0}$. (Fairtlough and Wainer [1998] credits this result to Parsons [1970], Mints [1973] and Takeuti [1987]). Hence to get a negative answer (to the above question) one must show using $R T_{2}^{2}$ that some computable but not primitive recursive function (such as the Ackermann function) is provably total. The functions provably total in $R C A_{0}+I \Sigma_{2}$ include the Ackermann function and far larger functions.

Question 13.3. Does the converse to Lemma 6.6 hold? It is known that $W K L_{0}$ is $\Pi_{1}^{1}$-conservative over $R C A_{0}+I \Sigma_{3}$. So a particular case of the above question is: Is every countable model of $R C A_{0}+I \Sigma_{3}$ an $\omega$-submodel of some countable model of $W K L_{0}+I \Sigma_{3}$ ?

A positive answer to the last question in Question 13.3 would imply every countable model of $R C A_{0}+I \Sigma_{3}$ is an $\omega$-submodel of some countable model of $W K L_{0}+I \Sigma_{3}+R T_{<\infty}^{2}$. Our current techniques can be used to show for all $n$, every countable topped model of $R C A_{0}+I \Sigma_{n}$ is an $\omega$-submodel of some countable model of $W K L_{0}+I \Sigma_{n}+R T_{<\infty}^{2}$ (this is an improvement on Theorem 11.2). A positive answer would also imply a positive answer to the following question. (For details of how this implication would go, see the proof of Lemma 9.6 and then use Lemma 6.6.) Call a theory $T$ a $\Pi_{2}^{1}$ theory if all of its axioms are $\Pi_{2}^{1}$ sentences.

Question 13.4. If $T_{0}$ and $T_{1}$ are $\Pi_{2}^{1}$-theories (in second order arithmetic) which are each $\Pi_{1}^{1}$-conservative over $T\left(R C A_{0}\right)$ is $T_{0}+T_{1}$ also $\Pi_{1}^{1}$-conservative over $T\left(R C A_{0}\right)$ ?

The above question can be answered negatively if we remove the restriction that the theories be $\Pi_{2}^{1}$-theories. One can show that every countable model of $R C A_{0}$ is an $\omega$-submodel of some countable model of $R C A_{0}+\neg$ Weak König's Lemma (we will leave the details as an exercise). Hence both Weak König's Lemma and its negation are $\Pi_{1}^{1}$ conservative over $R C A_{0}$ but clearly the conjunction of these two sentences is not.

Answers to the following questions would allow one to flesh out the above diagram and theorem.

Question 13.5. Does $R T_{2}^{2}$ imply Weak König's Lemma in $R C A_{0}$ ? Does $R T_{<\infty}^{2}$ imply Weak König's Lemma? 
Question 13.6. Does $S R T_{2}^{2}$ imply $R T_{2}^{2}$ ? (Does $S R T_{2}^{2}$ imply COH?) Does $S R T_{<\infty}^{2}$ imply $R T_{<\infty}^{2}$ ? (Does $S R T_{<\infty}^{2}$ imply COH)?

Question 13.7. Does $R T_{2}^{2}$ imply $I \Sigma_{2}$ ? Does $R T_{<\infty}^{2}$ imply $I \Sigma_{3}$ ?

Question 13.8. Does the "Chain or Anti-chain Condition" imply $R T_{2}^{2}$ ? (The Chain or Anti-chain Condition is the statement that every infinite partial order has an infinite chain or an infinite anti-chain. This statement follows easily from $R T_{2}^{2}+R C A_{0}$.) In Herrmann [n.d.] it is shown that there is a computable partial order of $\omega$ with no infinite $\Delta_{2}^{0}$ chain or anti-chain.

\subsection{Computability Theory Questions.}

Question 13.9. For each $\Delta_{2}^{0}$ set $A$ is there an infinite low set $X$ which is contained in or disjoint from A? Equivalently, does each stable computable 2-coloring of $[\mathbb{N}]^{2}$ have an infinite low homogeneous set? A relativizable positive answer would imply that $S R T_{2}^{2}$ is strictly weaker than $R T_{2}^{2}$ over $R C A_{0}$.

Question 13.10. For each $\Delta_{3}^{0}$ set $A$ is there an infinite low set which is contained in or disjoint from $A$ ?

Question 13.11. For each noncomputable set $C$ and each computable

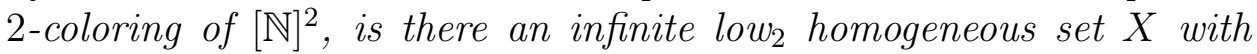
$C \mathbb{E}_{T} X$ ?

Question 13.12. For every 2-coloring $\mathcal{C}$ of $[\mathbb{N}]^{2}$ which is not of $P A$ degree is there an infinite homogeneous set $H$ such that $C \oplus H$ is not of PA degree? A relativizable positive answer will lead to a negative answer to Question 13.5 .

Question 13.13. What degrees $\mathbf{d}$ have the property that every 2coloring of $[\mathbb{N}]^{2}$ has an infinite homogeneous set of degree at most $\mathbf{d}$ ? (Clearly every degree $\mathbf{d}>>\mathbf{0}^{\prime}$ has this property, but the converse is false. Indeed Hummel and Jockusch [n.d.] has shown that there is a degree with the above property which is incomparable with $\mathbf{0}^{\prime}$.)

\section{REFERENCES}

Fairtlough, M. and Wainer, S. S. [1998]. Hierarchies of provably recursive functions, Handbook of proof theory, Vol. 137 of Stud. Logic Found. Math., North-Holland, Amsterdam, pp. 149-207. 13.3

Friedman, H. [1975]. Some systems of second order arithmetic and their use, pp. 235-242. 2

Friedman, H. [1976]. Systems of second order arithmetic with restricted induction, I, II (abstracts), Journal of Symbolic Logic 41: 557-559. 6.7 
Graham, R. L., Rothschild, B. L. and Spencer, J. H. [1980]. Ramsey theory, WileyInterscience Series in Discrete Mathematics, John Wiley \& Sons, Inc., New York. A Wiley-Interscience Publication. 1

Hájek, P. and Pudlák, P. [1993]. Metamathematics of first-order arithmetic, Perspectives in Mathematical Logic, Springer-Verlag, Berlin. 1, 2, 6, 11.

Herrmann, E. [n.d.]. Infinite chains and antichains in recursive partial orderings. To Appear. 13.8

Hirst, J. L. [1987]. Combinatorics in Subsystems of Second Order Arithmetic, PhD thesis, The Pennsylvania State University. 9.5

Hummel, T. L. [1994]. Effective versions of Ramsey's theorem: Avoiding the cone above $\mathbf{0}^{\prime}$, J. Symbolic Logic 59: 1301-1325. 3

Hummel, T. L. and Jockusch, Jr., C. G. [n.d.]. Generalized cohesiveness. To Appear in J. Symbolic Logic. 3, 7, 12.3, 13.13

Jockusch, Jr., C. G. [1968]. Semirecursive sets and positive reducibility, Trans. Amer. Math. Soc. 131: 420-436. 4

Jockusch, Jr., C. G. [1972]. Ramsey's theorem and recursion theory, J. Symbolic Logic 37: 268-280. 1, 2.5, 2, 12.1, 12.2

Jockusch, Jr., C. G. [1973]. Upward closure and cohesive degrees, Israel J. Math. 15: $332-335$. $4.1,12.4$

Jockusch, Jr., C. G. and Soare, R. I. [1972]. $\Pi_{1}^{0}$ classes and degrees of theories, Trans. Amer. Math. Soc. 173: 33-56. 2.1, 2, 8.1, 12.2

Jockusch, Jr., C. G. and Stephan, F. [1993]. A cohesive set which is not high, Math. Log. Quart. 39: 515-530. 1, 3.3, 3, 4.3, 4.5, 7, 9.5, 12.2, 12.4

Jockusch, Jr., C. G. and Stephan, F. [1997]. Correction to "a cohesive set which is not high", Math. Log. Quart. 43: 569.3

Kaye, R. [1991]. Models of Peano arithmetic, Vol. 15 of Oxford Logic Guides, The Clarendon Press Oxford University Press, New York. Oxford Science Publications. 1, 6, 11

Mints, G. E. [1973]. Quantifer-free and one quantifer systems, J. of Soviet Math. 1: $71-84.13 .3$

Mytilinaios, M. E. and Slaman, T. A. [1994]. On a question of Brown and Simpson. Preprint. 1, 11, 11.2

Odifreddi, P. [1989]. Classical Recursion Theory (Volume I), North-Holland Publishing Co., Amsterdam. 12.2

Parsons, C. [1970]. On a number theoretic choice schema and its relation to induction, Intuitionism and Proof Theory (Proc. Conf., Buffalo, N.Y., 1968), North-Holland, Amsterdam, pp. 459-473. 13.3

Posner, D. B. and Robinson, R. W. [1981]. Degrees joining to 0', J. Symbolic Logic 46(4): 714-722. 12.2

Ramsey, F. P. [1930]. On a problem in formal logic, Proc. London Math. Soc. (3) 30: $264-286$. 1

Scott, D. [1962]. Algebras of sets binumerable in complete extensions of arithmetic, Recursive Function Theory, number 5 in Proceedings of Symposia in Pure Mathematics, American Mathematical Society, Providence, R.I., pp. 117-121. 8.16

Seetapun, D. and Slaman, T. A. [1995]. On the strength of Ramsey's theorem, Notre Dame J. Formal Logic 36(4): 570-582. Special Issue: Models of arithmetic. 1 . 1. 2.7, 2.8, 2.9, 2.10, 5.1, 10.2 
Simpson, S. G. [1977]. Degrees of unsolvability: a survey of results, in J. Barwise (ed.), Handbook of Mathematical Logic, North-Holland, Amsterdam, pp. 11331142. 4

Simpson, S. G. [1999]. Subsystems of second order arithmetic, Perspectives in Mathematical Logic, Springer-Verlag, Berlin. 2, 2.2, 2, 2.6, iv, 6, 6, 6, 7.2, 8.1, 8.4. 8.5

Soare, R. I. [1987]. Recursively Enumerable Sets and Degrees, Perspectives in Mathematical Logic, Omega Series, Springer-Verlag, Heidelberg. 1

Specker, E. [1971]. Ramsey's Theorem does not hold in recursive set theory, Logic Colloquium; 1969 Manchester, pp. 439-442. 2, 2.3, 2.4

Spector, C. [1956]. On the degrees of recursive unsolvability, Ann. of Math. (2) 64: $581-592$. 12.2

Takeuti, G. [1987]. Proof theory, Vol. 81 of Studies in Logic and the Foundations of Mathematics, second edn, North-Holland Publishing Co., Amsterdam. With an appendix containing contributions by Georg Kreisel, Wolfram Pohlers, Stephen G. Simpson and Solomon Feferman. 13.3

Department of Mathematics, University of Notre Dame, Notre DAME, IN 46556-5683

E-mail address: Peter.Cholak.1@nd.edu

Department of Mathematics, University of Illinois at UrbanaChampaign, 1409 W. Green Street, Urbana, Illinois 61801-2975

E-mail address: jockusch@math.uiuc.edu

Department of Mathematics, University of California, Berkeley, CA 94720-3840

E-mail address: slaman@math.berkeley.edu 